Universidad Autónoma Metropolitana Iztapalapa Div. de Ciencias Básicas e Ingeniería Dpto. de Física

\title{
Estudio de las Escalas de Tiempo en la Dinámica Estocástica Transitoria de Sistemas Inestables Rotacionales
}

\author{
Me
Tesis que presenta \\ M. en Fís. Pedrol.Orea
}

Para la obtención del grado de Doctor en Ciencias

Asesor:

Dr. José Inés Jiménez Aquino

Junio del 2001 


\section{A mis padres \\ Juana Orea Evodio Vázquez}

Por haberme dado la vida.

A mi esposa e hijos

\section{Estela}

Karen P. Hafid

Por la felicidad que me brindan.

i A Mi PUEblo Sta. ROSA ! 


\section{Agradecimientos}

Mis más sinceros agradecimientos a mi asesor de tesis, Dr. José Inés Jiménez Aquino por su inagotable paciencia durante el desarrollo de este trabajo de tesis.

A los Drs: Leopoldo García Colín Scherer, Eduardo Pi na Garza, José Luis del Río Correa, Raúl Montes de Oca Machorro, Víctor Romero Rochín y Olegario Alarcón Waess; por sus valiosas observaciones y críticas en la revisión de esta tesis.

Aprovecho también la oportunidad para agradecer a todos los profesores que me impartieron clases durante toda mi formación profesional.

A mis hermanos, a sus respectivas esposas e hijos y en especial a mis hermanas Rosa y Consuelo; por su apoyo económico y moral que siempre me han brindado.

A mis suegros, por el apoyo invaluable con el que siempre he contado.

En especial a Jesus Rodríguez y Angelina Jiménez, por haberme brindado su confianza y apoyo durante esta etapa de mi vida.

A todos mis amigos, que creyeron en mi. Y en especial a los de la vecindad de Don "Memo", que hicieron un momento agradable de todo este tiempo.

Al CONACyT por la ayuda económica. 


\section{Nomenclatura}

- DTP: Distribución de los Tiempos de Paso

226001

- DCM: Desplazamiento Cuadrático Medio

- ETL: Ecuación Tipo Langevin

- FETCD: Formulación Estándar de la Teoría Cuasideterminista

- FMTCD: Formulación Matricial de la Teoría Cuasideterminista

- PB: Partícula Browniana

- RBG: Ruido Blanco Gaussiano

- RCG: Ruido de Color Gaussiano

- TCD: Teoría Cuasi-Determinista

- TRNL: Tiempos de Relajación No Lineales

- TP: Tiempos de Paso

- VAG: Variable Aleatoria Gaussiana 


\section{Indice}

1 Introducción 1

1.1 Motivación y aspectos generales . . . . . . . . . . . . . 1

1.1 .1 Contexto . . . . . . . . . . . . . . . . 1

1.1 .2 Fenomenología . . . . . . . . . . . . . . . 2

1.1 .3 Metodología . . . . . . . . . . . . . . . . . . 3

1.1.4 Clasificación de los procesos dinámicos . . . . . . . . . . 5

1.2 Análisis de las escalas de tiempo . . . . . . . . . . . . . . 7

1.2 .1 Movimiento Browniano . . . . . . . . . . . . . . . . 7

1.2 .2 Ecuación de Langevin . . . . . . . . . . . . . . . . 8

1.2.3 Decaimiento de sistemas inestables . . . . . . . . . . . 11

1.3 Objetivo Principal . . . . . . . . . . . . . . . . . . 14

2 Caracterización dinámica de sistemas inestables. Formulación Estándar 22

2.1 Definición general de estados inestables . . . . . . . . . . . . . 22

2.2 Análisis cualitativo de la teoría cuasi-determinista . . . . . . . . . . 23

2.3 Formulación Estándar de la teoría cuasi-determinista . . . . . . . . . . 24

2.3.1 Efecto de condiciones iniciales distribuidas . . . . . . . . . 24

2.3.2 Efecto de condiciones iniciales fijas . . . . . . . . . . . . 29

2.3.3 Tiempo de Paso inducidos por ruido blanco gaussiano . . . . . . . 30

2.3.4 Tiempo de Paso inducidos por ruido de color gaussiano . . . . . . . 30

2.4 Los tiempos de relajación no lineales y la teoría cuasi-determinista . . . . . 33

2.4.1 Caracterización dinámica de sistemas inestables en el régimen lineal 34

2.4.2 Caracterización dinámica de sistemas inestables en el régimen no lineal . . . . . . . . . . . . . . . . . . . . 35

2.4.3 Los TRNL inducidos por ruido blanco gaussiano . . . . . . . . 36

2.4.4 Los TRNL inducidos por ruido de color gaussiano . . . . . . . . 37

2.4.5 Modelo de Landau . . . . . . . . . . . . . . . . . . . 37

2.5 Formulación Estándar de TCD en presencia de fuerza externa constante . . 38

2.5.1 Sistemas de dos variables . . . . . . . . . . . . . . . . 41

2.5.2 Sistemas de tres variables $\ldots \ldots \ldots \ldots \ldots \ldots$

3 Caracterización dinámica de sistemas inestables rotacionales. Formulación Matricial $\quad 43$

3.1 Formulación Matricial de la teoría cuasi-determinista . . . . . . . . 4 
3.1.1 Ruido blanco gaussiano . . . . . . . . . . . . . . . . . . . . . 44

3.1 .2 Ruido de color gaussiano . . . . . . . . . . . . . . . . . . . . 48

3.2 Sistemas rotacionales de $\operatorname{dos}$ variables . . . . . . . . . . . . . . 51

3.3 Sistemas rotacionales de tres variables . . . . . . . . . . . . . 53

3.4 Justificación del formalismo generalizado de TCD . . . . . . . . 56

4 Caracterización dinámica de sistemas inestables rotacionales en presen$\begin{array}{ll}\text { cia de fuerzas externas } & 60\end{array}$

4.1 Formulación Matricial de la teoría cuasi-determinista . . . . . . . . . . . 61

4.1.1 En presencia de fuerza externa constante . . . . . . . . . . . 61

4.1.2 En presencia de fuerza externa dependiente del tiempo . . . . . . 63

4.2 Sistemas rotacionales de dos variables . . . . . . . . . . . . . . 64

4.2.1 En presencia de fuerza externa constante . . . . . . . . . . . 64

4.2.2 En presencia de fuerza externa dependiente del tiempo $\ldots \ldots . .69$

4.3 Sistema Láser . . . . . . . . . . . . . . . . . . . . . . . . . . . 71

4.3.1 En presencia de campo externo constante . . . . . . . . . . 73

4.3.2 Receptor de Salida . . . . . . . . . . . . . . . . . . 75

4.3.3 En presencia de campo externo dependiente del tiempo . . . . . . 76

4.4 Sistemas rotacionales de tres variables $\ldots \ldots \ldots \ldots . \ldots 78$

$\begin{array}{llr}5 & \text { Conclusiones y perspectivas } & 81\end{array}$

A Propiedades de la matriz antisimétrica W $\quad 86$

A.1 Demostración de $\mathrm{e}^{\mathbf{W} t}=\mathbf{R}(t) \ldots \ldots \ldots \ldots \ldots$. . . . . . . 86

A.2 Demostración de $\mathbf{W} \mathbf{x}=\vec{w} \times \mathbf{x} \ldots \ldots \ldots \ldots . \ldots . \ldots . \ldots 8$

A.3 La matriz $\mathbf{W}$ para el caso de tres variables . . . . . . . . . . . 88

B Fundamentos de la dinámica rotacional de Langevin 90

B.1 Relaciones de Reciprocidad de Onsager-Casimir . . . . . . . . . . . . . 90

C Cálculo Analítico de las series (2.84) y (2.92) 94

C.1 Dos variables . . . . . . . . . . . . . . . . . . . . . . . . . 94

C.2 Tres variables $\ldots \ldots \ldots \ldots \ldots$

$\begin{array}{ll}\text { Referencias } & 96\end{array}$ 


\section{Capítulo 1}

\section{Introducción}

\subsection{Motivación y aspectos generales}

\subsubsection{Contexto}

Hasta inicios del siglo XX, toda la física estaba formulada en términos de ecuaciones diferenciales deterministas que parecían ser capaces de predecir con mucha exactitud el comportamiento de los sistemas.

Con la Mecánica Cuántica se introduce el concepto de probabilidad en una observación física, es decir, que "la misma causa provoca diferentes efectos, y cada uno de estos efectos tiene una probabilidad de suceder".

La rama de la física que se encarga de estudiar las propiedades físicas en el bulto a partir del comportamiento dinámico de sus elementos microscópicos, es la Mecánica Estadística; la cual se divide en dos partes fundamentales: Equilibrio y No Equilibrio. La mecánica estadística de equilibrio está satisfactoriamente fundamentada, en el sentido que así es aceptado por la mayoria de sus investigadores, mediante la teoría de conjuntos representativos. En cambio la fundamentación de la mecánica estadística de no equilibrio no es en absoluto satisfactoria, debido a múltiples causas entre las que podríamos destacar la dificultad de hacer una buena clasificación de los diferentes procesos de no equilibrio. La diferencia que hay entre la mecánica estadística de equilibrio y no equilibrio es la existencia de potenciales termodinámicos. Para el caso de equilibrio con solo conocer dichos potenciales podemos hallar, en principio, cualquier propiedad macróscopica. Por el contrario, para el caso de no equilibrio todavía nos estamos preguntando si tales potenciales existen. 
Sin querer en modo alguno ser exhaustivo vamos a presentar una posible clasificación de los procesos fuera de equilibrio, los cuales podemos clasificar en tres grupos que son:

a) Evolución dinámica entre estados de equilibrio.

b) Evolución dinámica entre estados estacionarios en general.

c) Estados estacionarios de no equilibrio.

Los dos primeros involucran dependencia temporal. En el tercer grupo tenemos los estados independientes del tiempo, pero que no son de equilibrio debido a ciertas condiciones de contorno, presencia de gradientes, flujos, etc.

Existen también muchos tratamientos correctos de diversas situaciones de No Equilibrio pero que dependen de la situación concreta y no son generalizables a otros casos. Por otra parte, si utilizamos el recurso de resolver las ecuaciones de Newton mediante el uso de computadoras, no es una tarea fácil de hacer, por lo que este método no nos ayuda a resolver sistemas de mucho interés. Incluso la descripción de los estados de no equilibrio usando herramientas de equilibrio, está sujeto a fuerte discusión.

En este trabajo no vamos a tratar de encontrar una teoría de la evolución dinámica, sino que vamos a tomar una de las dinámicas ya aceptadas en la rica fenomenología de no equilibrio, que es "la Dinámica de Langevin" y sus correspondientes generalizaciones. Esta dinámica nos permite clasificar los procesos dinámicos que únicamente pueden aparecer debido a la presencia de fluctuaciones.

\subsubsection{Fenomenología}

Desde hace un tiempo viene desarrollándose una gran actividad en el estudio de sistemas fuera del equilibrio, fundamentalmente mediante el uso de simulación a través del uso de computadoras. También hay que añadir la cada día más abundante proliferación de experimentos típicos de relajación dinámica de sistemas sometidos a fluctuaciones, tales como: el estudio de la Dinámica de Transiciones de Fase desde diferentes puntos de vista [51,34, 16]; la relajación de inestabilidades ópticas [3], hidrodinámicas [56], químicas [60], etc. De todos estos estudios ha surgido una muy rica fenomenología que pone de manifiesto tanto la importancia de las fluctuaciones, como el comportamiento no lineal de los sistemas cerca de los estados inestables $[13,15,49,54]$. Al tratar de poner orden en toda esta fenomenología surgen diversas opciones a considerar; de todas las posibilidades. 
nos vamos a fijar en aquellas situaciones dinámicas donde las fluctuaciones juegan un papel primordial, pero sin olvidar las no linealidades del sistema. ¿Cuál es la motivación para esta elección?. La razón es muy simple. La clasificación de los procesos dinámicos en los que las fluctuaciones son fundamentales es muy reducida, y se deja ordenar utilizando únicamente criterios que llamaríamos mecánico-clásicos. Además las diferentes clases que aparecen representan situaciones físicas de mucho interés y contienen información sobre la dinámica del sistema difícil de vislumbrar por otros métodos. Para cada una de estas clases buscamos una escala temporal definiendo un tiempo característico de una manera simple. Esta información es fundamental, pues cada proceso dinámico tiene pocas escalas de tiempo posibles, independientemente de cómo se observen, por lo que si logramos hallarlas de una forma u otra, tendremos un conocimiento global dinámico del sistema.

\subsubsection{Metodología}

Puesto que las fluctuaciones van a jugar un papel predominante; la metodología matemática que nos permitirá desarrollar su estudio es la Teoría de los Procesos Estocásticos . Esta metodología ocupa un papel cada vez más preponderante en la Mecánica Estadística de No Equilibrio, como puede apreciarse en la abundante proliferación de monografias específicas $[63,57,60,15,49,13,18]$.

Los Procesos Estocásticos pueden estudiarse a partir de diferentes marcos matemáticos: ecuaciones de movimiento para las diferentes probabilidades estadísticas asociadas a un proceso estocástico (Ecuación Maestra, Ecuación de Fokker-Planck, etc.); a partir de ecuaciones diferenciales estocásticas que obedecen dichos procesos (Ecuaciones de Langevin, generalizadas o no). En este proyecto vamos a escoger el segundo punto de partida, por la sencilla razón de que las ecuaciones diferenciales estocásticas tienen una interpretación física simple que ayudará a comprender mejor los mecanismos físicos involucrados.

Las fluctuaciones son una característica intrínseca de todos los sistemas reales. Estas son inevitables en todos los sistemas naturales. La intensidad de estas fluctuaciones que llamaremos internas, se mide mediante un parámetro que denotaremos por $D$ o $Q$. Hay varias clases de fluctuaciones internas, por ejemplo, en los fenómenos térmicos y químicos, la intensidad de las fluctuaciones será proporcional a la temperatura absoluta; y en los fenómenos atómicos o nucleares, a la constante universal de Planck; y en los sistemas 
químicos finitos al inverso del volumen del sistema o del número de moléculas que intervienen en la reacción. Las fluctuaciones internas pueden dar origen, por ejemplo, a nuevos estados macroscópicos estables o a que se desplacen los puntos de inestabilidad que delimitan las distintas fases. También estas fluctuaciones pueden producirse y controlarse en el laboratorio, lo que permite un estudio más preciso de su influencia en el comportamiento del sistema. Además, las fluctuaciones internas juegan un papel relevante en la relajación de estados inestables y en la transición espontánea entre estados estables. Exceptuando estos dos fenómenos, las fluctuaciones internas pueden despreciarse sin problema alguno; en los sistemas muy grandes y siempre que estemos lejos de una transición de fase.

Hay una segunda clase de fluctuaciones, que podemos llamar fluctuaciones externas, debidas a la estocasticidad del medio que rodea al sistema o bien a los parámetros de control del mismo. La diferencia principal que hay entre las fluctuaciones externas y las internas; estriba en que las primeras son controlables $y$, por ello, no pueden despreciarse tan fácilmente. Se trata de fluctuaciones que inducen cambios sustanciales con respecto a las fluctuaciones internas.

La descripción fenomenológica convencional que toma en cuenta fluctuaciones externas consiste esencialmente en empezar con una ecuación de movimiento determinista para la variable que representa al sistema. Estas ecuaciones son similares muchas veces a las que aparecen en cinética química y dependen de los parámetros externos, tales como: potenciales eléctricos, concentraciones de los reactantes, catalizadores, etc. Las fluctuaciones externas se introducen a través de estos parámetros, suponiendo que estas fluctúan alrededor de cierto valor medio. La ecuación de movimiento de partida se convierte ahora en una ecuación diferencial estocástica, cuyas características pueden tener distintos grados de complejidad. Estas fluctuaciones o también llamados ruidos tienen propiedades estadísticas propias.

Los dos tipos de ruido que se utilizan con mayor frecuencia en la literatura y que sirven de base en nuestro estudio son:

Ruido Blanco Gaussiano (RBG). Los valores que toma el ruido siguen una ley de distribución normal, y no está correlacionado salvo a tiempos iguales, es decir, que no tiene memoria. Este proceso es el más utilizado por su sencillez matemática, sólo necesita un parámetro para su descripción y es la intensidad del mismo, denotada por $D \circ Q$. S recurre a él cuando no se conoce realmente bien el ruido a que está sometido el sistema. así 
como para representar las fluctuaciones de origen interno (por ejemplo, en el movimiento browniano).

Ruido Gaussiano de Color (RGC). Este ruido sí está correlacionado consigo mismo a tiempos distintos, es decir, que tiene memoria. Y además posee un parámetro más, $\tau$, llamado tiempo de correlación. Se trata de una fluctuación muy cercana a los ruidos reales, por esta razón se recurre a ella para representarlos. La descripción matemática de los procesos influidos por este ruido es muy compleja. La velocidad de la partícula Browniana libre sigue este proceso.

Existen otros tipos de ruido tales como: ruido blanco de Poisson y ruido dicotómico, que son importantes en otras situaciones físicas. En este estudio no se van a trabajar con estos ruidos.

\subsubsection{Clasificación de los procesos dinámicos}

A continuación vamos a clasificar los precesos dinámicos que pueden ocurrir en la evolución de un sistema, situado inicialmente en un estado que no coincide con el estado estacionario que le corresponde. Es decir, vamos a clasificar los diferentes procesos que pueden ocurrir en las evoluciones dinámicas $a$ ) y $b$ ) introducidos en la Subsec. 1.1.1. Para ello, empezaremos con ejemplos concretos de situaciones físicas y posteriormente seleccionaremos a qué clase de proceso dinámico corresponde.

Tomemos un sistema paramagnético $\left(T>T^{0}\right)$ y disminuyamos rápidamente la temperatura $\left(T<T^{0}\right)$. El sistema evolucionará en la dirección del nuevo estado de equilibrio ferromagnético que le corresponde ( proceso dinámico a) de la Subsec. 1.1.1. O bien. si variamos súbitamente el parámetro de bombeo de un Láser éste pasará a un estado de emisión estimulada diferente del inicial (proceso dinámico b) de la Subsec. 1.1.1. En ambos casos el sistema es inicialmente inestable frente a fluctuaciones infinitésimales, sin necesidad de especificar su origen por el momento. A este proceso de relajación se le clasifica como "Relajación de un Estado Inestabale". Este es el tipo de proceso de relajación que vamos a estudiar en esta tesis.

Un segundo caso, se tiene cuando el sistema después de permanecer un tiempo apreciable en un estado inicial que parecía estacionario súbitamente cambia a otro y permanec $\epsilon^{\prime}$ en él cierto tiempo del mismo orden de mágnitud que el primer estado, volviendo otra ve\% 
al estado inicial y repitiendo el ciclo indefinidamente. Se dice entonces que estos estados son inestables frente a fluctuaciones finitas y el proceso se clasifica como "Relajación de un Estado Metaestable o Biestable".

Un tercer caso, surge en el límite en que desaparece, de forma continua, uno de los estados del caso metaestable. Al estado que va a desaparecer se le llama estado marginal y el proceso dinámico se denomina "Relajación de un Estado Marginal". Las fluctuaciones que originan esta evolución son infinitésimales.

Existe un cuarto caso, este se logra cuando el sistema tiene un único estado estacionario estable y variando los parámetros de control lo alejamos de dicho estado sin introducir nuevos estados estables. En este caso, el sistema vuelve al estado que le corresponde de una forma que llamaríamos suave, sin necesidad de las fluctuaciones. Al proceso dinámico se le denomina "Relajación de un Estado Estable". Esta relajación esta muy bien descrita y estudiada por la Mecánica Estadística de No Equilibrio Lineal.

Estos cuatro procesos de relajación tienen lugar en una escala de tiempo característica que dependen de los parámetros del sistema y de las fluctuaciones.

Esta clasificación es ciertamente muy general, pero suficiente para tener una idea de qué tan importante y abundante es el estudio de la relajación dinámica de sistemas. Antes de empezar su estudio hay que delimitar qué modelos o situaciones concretas y en qué circunstancias se van a estudiar. Pueden considerarse modelos con diferentes grados de libertad, en particular hay que distinguir entre los de dimensionalidad diferente de cero (descripción mediante Teorías de Campos) o los de dimensionalidad cero (número finito de variables). En el primer caso, la relajación tiene lugar localmente produciéndose estructuras espaciales transitorias. En el segundo caso, la relajación ocurre globalmente.

En este trabajo nos vamos a fijar en las situaciones cero-dimensionales a fin de simplificar el problema y poder obtener resultados analíticos que nos permitan ilustrar los mecanismos dinámicos más elementales, que sean comunes a cualquier otra situación más compleja. En las situaciones de mayor complejidad las aproximaciones del cálculo podrían ocultar aspectos físicos de interés, para nuestro análisis, ésta es la razón por la que simplificamos el problema. 


\subsection{Análisis de las escalas de tiempo}

\subsubsection{Movimiento Browniano}

Al estudiar el movimiento de una partícula muy pequeña (Partícula Browniana: PB) en un fluido se observó que su movimiento era totalmente errático y permanente, es decir, la partícula nunca deja de moverse en el fluido ${ }^{1}$. Albert Einstein en 1905 dio la explicación del movimiento browniano a través de la hipótesis molecular, que consiste en que el movimiento errático continuo de la $\mathrm{PB}$ se debe al enorme golpeteo $\left(10^{21}\right)$ de las moleculas del fluido con la partícula browniana [11]; además planteó la posibilidad de medir experimentalmente el Desplazamiento Cuadrático Medio (DCM) de la PB, para así poder comprobar la hipótesis molecular. Este experimento lo llevo a cabo Jean Perrin et. al. entre los años 1908-1911 [46]. La teoría de Einstein consiste en derivar una descripción probabilística válida para todo un conjunto de partículas, en lugar de seguir en el tiempo la complicada trayectoria de una sola de ellas $[63,12,15]$. El método se basa en tres hipótesis a saber:

1.- El movimiento errático está causado por los constantes impactos de las moléculas del fluido sobre la partícula browniana.

2.- El movimiento de cada partícula es independiente de las demás.

3.- La descripción del proceso ha de ser probabilística.

Bajo estas condiciones Einstein mostró que la densidad de probabilidad satisface la siguiente ecuación diferencial parcial, dada por

$$
\frac{\partial f}{\partial t}=D \frac{\partial^{2} f}{\partial x^{2}}
$$

Esta ecuación se conoce en la literatura como la ecuación de difusión, donde D corresponde al coeficiente de difusión. Si la condición inicial es que todas las partículas están localizadas en $x=0$ y $f(x, t=0)=n \delta(x)$, entonces la solución de esta ecuación es

$$
f(x, t)=\frac{n}{(4 \pi D t)^{1 / 2}} \exp \left(-\frac{x^{2}}{4 D t}\right) .
$$

\footnotetext{
${ }^{1}$ Este movimiento fue estudiado por vez primera por el botánico inglés Robert Brown en 1828, puesto que años antes ya habia sido observado este fenómeno por otros científicos, entre ellos: F.W. von Gleichen. J.T. Needham.
} 


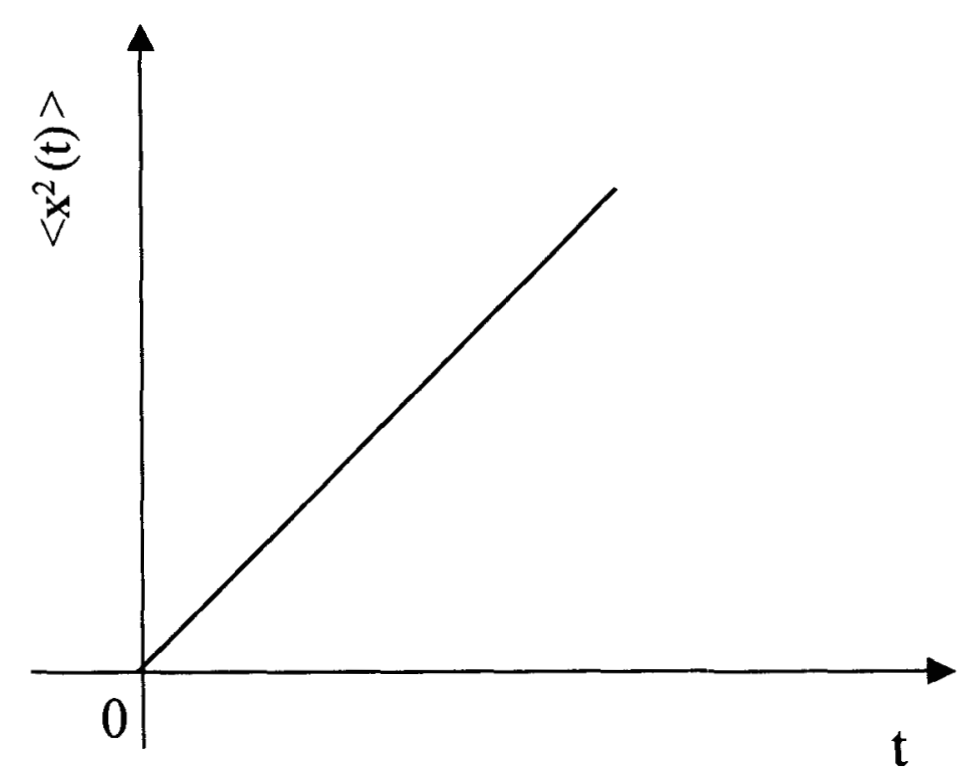

Fig. 1.1: Desplazamiento cuadrático medio para la partícula browniana

Como podemos ver esta expresión tiene la forma de una distribución gaussiana. Por lo que el promedio $\langle x(t)\rangle=0$ y su desplazamiento cuadrático medio es igual a

$$
\left\langle x^{2}(t)\right\rangle=2 D t
$$

La interpretación de este resultado es que el DCM incrementa linealmente con el tiempo, como se puede ver en la Fig. 1.1. Como ya se menciono anteriormente este resultado fue comprobado experimentalmente por J. Perrin [46], entre otros.

\subsubsection{Ecuación de Langevin}

Años después, Paul Langevin (1908) estudió este mismo problema, presentando un nuevo método el cual fue completamente diferente al de Einstein [35]. La propuesta de Langevin fue estudiar la trayectoria de una sola partícula a través del fluido, para ello dividió a la fuerza total que siente la particula browniana, en una fuerza sistemática y una fuerza fluctuante. La fuerza sistemática, que para el caso de una esfera de radio a y $M \gg m$ (siendo $M$ la masa de la PB y $m$ la masa de las moléculas del fluido), moviéndose a velocidades no tan grandes en un fluido de viscosidad $\eta$, viene dada por la ley de Stokes $F_{f}=-\gamma v$ donde el subíndice $f$ indica fricción y $v=\frac{d x}{d t}$ la velocidad. El coeficiente de fricción para la partícula browniana esférica de radio $a$ es igual a $\gamma=6 \pi \eta a$. La fuerza 
estocática o ruido $\xi(t)$ representa los choques incesantes de las moléculas del fluido $\left(10^{21}\right.$ choques por segundo) con la PB. Con toda esta información Langevin propuso que la segunda ley de Newton para la PB en ausencia de fuerza externa $(V(x)=0$, siendo $V(x)$ el potencial) satisface la siguiente ecuación diferencial

$$
M \frac{d^{2} x}{d t^{2}}=-\gamma \frac{d x}{d t}+\xi(t)
$$

que es precisamente una ecuación diferencial estocástica debido a la presencia del término fluctuante $\xi(t)$, cuya solución en promedio requiere de las propiedades estadísticas de dicha fuerza.

Para obtener las propiedades estadísticas de la PB Langevin resolvió el problema de la siguiente forma. Si multiplicamos a la Ec. (1.4) por $x$, agrupamos términos y hacemos promedios sobre $\xi(t)$, nos queda

$$
\frac{M}{2} \frac{d^{2}}{d t^{2}}\left\langle x^{2}\right\rangle-M\left\langle\dot{x}^{2}\right\rangle=-\frac{\gamma}{2} \frac{d}{d t}\left\langle x^{2}\right\rangle+\langle x \xi(t)\rangle .
$$

Se hace a continuación dos importates hipótesis

$$
M\left\langle\dot{x}^{2}\right\rangle=K_{B} T, \quad\langle x(t) \xi(t)\rangle=\langle\xi(t)\rangle\langle x(t)\rangle=0 .
$$

En primer lugar, suponer que el promedio de la energía cinética es igual a $K_{B} T$, donde $K_{B}$ es la constante de Boltzmann y $T$ es la temperatura del fluido, implica haber alcanzado el equilibrio térmico (Teorema de Equipartición de la Energía). La segunda hipótesis nos dice que no hay correlación entre la fuerza fluctuante y la posición de la PB, debido a la gran irregularidad de la fuerza estocástica. El hacer uso de estas dos hipótesis implica asignarle propiedades a la función desconocida $\xi(t)$.

De esta manera, la ecuación se reduce a

$$
\frac{d^{2}}{d t^{2}}\left\langle x^{2}\right\rangle=-\frac{\gamma}{M} \frac{d}{d t}\left\langle x^{2}\right\rangle+\frac{2}{M} K_{B} T,
$$

que se puede resolver fácilmente, obteniendose

$$
\left\langle x^{2}(t)\right\rangle-\left\langle x^{2}(0)\right\rangle=\frac{2 K_{B} T}{\gamma}\left[\tau\left(e^{--\frac{1}{\tau} t}-1\right)+t\right],
$$

tal que $\tau \equiv \frac{M}{\gamma} \simeq 10^{-8} s$, siendo $\tau$ el tiempo de relajación. Esta ecuación nos permite hacer un análisis para tiempos cortos y para tiempos largos del problema. 


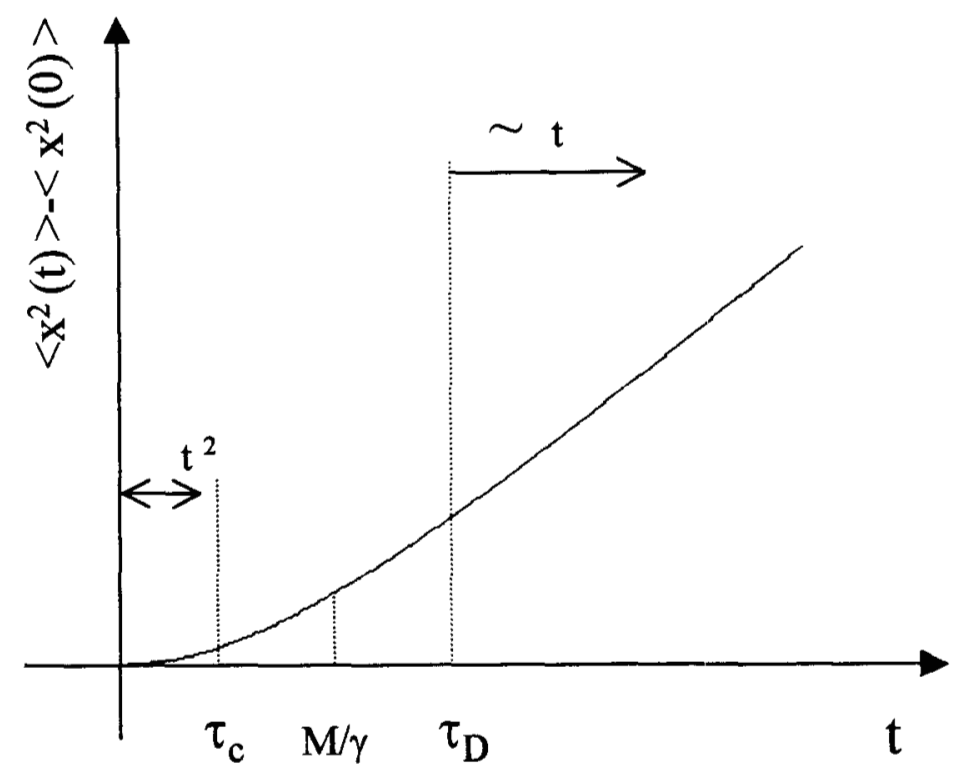

Fig. 1.2: Desplazamiento cuadrático medio para la partícula browniana

i) Tiempos Cortos $t \ll \tau$

$$
\left\langle x^{2}(t)\right\rangle-\left\langle x^{2}(0)\right\rangle \rightarrow 2 D t^{2}
$$

ii) Tiempos Largos $t \gg \tau$

$$
\left\langle x^{2}(t)\right\rangle-\left\langle x^{2}(0)\right\rangle \rightarrow 2 D t
$$

donde $D \equiv \frac{K_{B} T}{\gamma}$. Por lo tanto en el régimen de tiempos largos la expresión (1.10) recupera el resultado de Einstein; y en el régimen de tiempos cortos se tiene un comportamiento 3
6
3
3
3
6
6 parabólico que corresponde a la de la partícula libre. En la Fig. 1.2 se muestra la gráfica del DCM, de la PB donde podemos observar que en el régimen de tiempos cortos se tiene una curva parabólica que representa al régimen balístico de partícula libre; y para tiempos largos la gráfica muestra un comportamiento lineal igual al de la Fig. 1.1, el cual corresponde al régimen difusivo de acuerdo con la descripción de Einstein. En este régimen de tiempos largos, la partícula browniana siente la presencia del fluido a través de las constantes colisiones con las moléculas de dicho fluido.

En base a estos resultados decimos que en el esquema de Langevin hemos obtenido más información que con el enfoque de Einstein, que sólo da información en el régimen difusivo. En el esquema de Langevin, el haber propuesto la hipótesis de equilibrio térmico para la variable $\dot{x}(t)$, significa que esta variable alcanza el equilibrio antes que $x(t)$ de 
manera que la solución hallada no sólo es válida para tiempos largos, como la solución de Einstein, sino que también nos da información a tiempos cortos.

\subsubsection{Decaimiento de sistemas inestables}

Siguiendo las ideas de la sección anterior, estudiemos ahora el proceso de decaimiento de los sistemas inestables inducidos por la presencia de fluctuaciones estocásticas. Este fenómeno es naturalmente distinto al movimiento browniano, sin embargo un análisis similar de las escalas de tiempo de dicho proceso de decaimiento puede realizarse de forma análoga como en el caso del moviento browniano.

Para este propósito vamos a partir de ahora en adelante, de una ecuación similar a la ecuación de Langevin con las siguientes características: En nuestra descripción vamos a considerar que el término de inercia $M \frac{d^{2} x}{d t^{2}} \simeq 0$ y la introducción de otra fuerza sistemática derivable de un potencial ${ }^{2}$. La ecuación a la que nos estamos refiriendo en el caso de una variable, tiene entonces la siguiente estructura matemática

$$
\frac{d x}{d t}+\frac{d V(x)}{d x}=\xi(t)
$$

donde $V(x)$ es el potencial cuya fuerza sistemática asociada es $F=-\frac{d V(x)}{d x}$. La Ec. (1.11) describe entonces un sistema sobreamortiguado con fluctuaciones estocásticas. La variable $x$ no representa necesariamente la posición, sino cualquier otra variable física de interés que no sea la velocidad, como por ejemplo el campo eléctrico del sistema Láser, etc. La Ec. (1.11), se conoce en la literatura como la Ecuación Tipo Langevin (ETL) y es una ecuación diferencial estocástica cuya solución en promedio requiere de las propiedades estadísticas de la fuerza fluctuante o ruido $\xi(t)$.

Consideremos ahora el caso en el que el potencial es de la forma $V(x)=-\frac{a}{2} x^{2}$, con $a>0$ tal que la fuerza sistemática asociada es una fuerza lineal, dada por $F=a x$. Este potencial es una parábola invertida cuyo vértice coincide con el origen de coordenadas de la Fig. 1.3; el cual corresponde al estado inestable del potencial. Así que, en el proceso de decaimiento del estado inestable supondremos que, inicialmente el sistema se encuentra localizado en el origen de coordenadas y que por efecto de pequeñas fluctuaciones decaerá cuesta abajo del potencial. En este caso la ecuación tipo Langevin puede escribirse de la

\footnotetext{
${ }^{2}$ Esto no necesariamente ocurre en los sistemas rotacionales, como se verá más adelante
} 


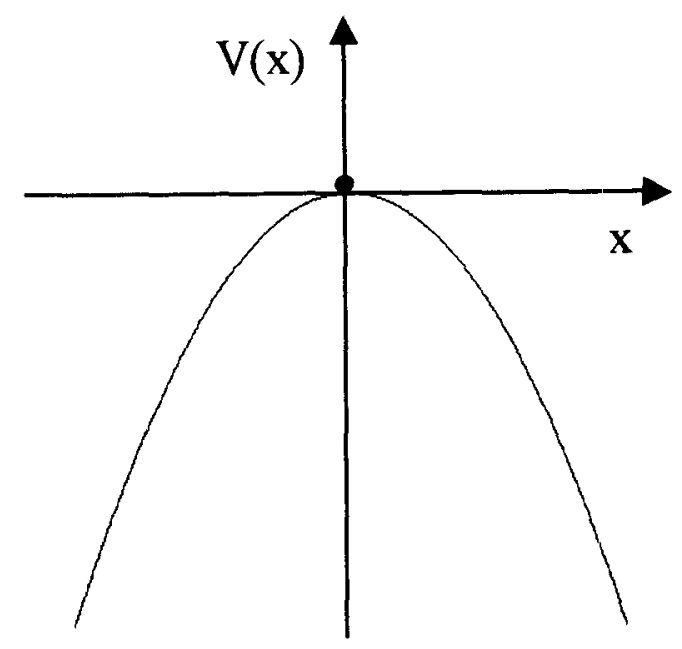

Fig. 1.3: Potencial $V(x)=-\frac{1}{2} a x^{2}$, para la visualización mecánica de un proceso de decaimiento de un estado inestable inicial

siguiente forma

$$
\dot{x}=a x+\xi(t) \text {. }
$$

Partiendo de la Ec. (1.12) hagamos ahora el análisis de las escalas de tiempo siguiendo las ideas de la sección anterior. Para este propósito requerimos de las propiedades de la fuerza fluctuante $\xi(t)$ que por simplicidad supondremos que es un ruido blanco gaussiano; cuyo valor medio es igual a $\langle\xi(t)\rangle=0$ y su correlación

$$
\left\langle\xi(t) \xi\left(t^{\prime}\right)\right\rangle=2 D \delta\left(t-t^{\prime}\right)
$$

donde $\mathrm{D}$ es la intensidad del ruido.

La solución de la Ec. (1.12) está dada por

$$
x(t)=h(t) e^{a t}
$$

donde

$$
h(t)=x(0)+\int_{0}^{t} e^{-a t^{\prime}} \xi\left(t^{\prime}\right) d t^{\prime} .
$$

El primer término del lado derecho de la igualdad (1.15) se refiere a las condiciones iniciales del sistema, que por simplicidad supondremos que $x(0)=0$.

El primer momento de la Ec. (1.14) es igual a

$$
\langle x(t)\rangle=\langle h(t)\rangle e^{a t}=0,
$$




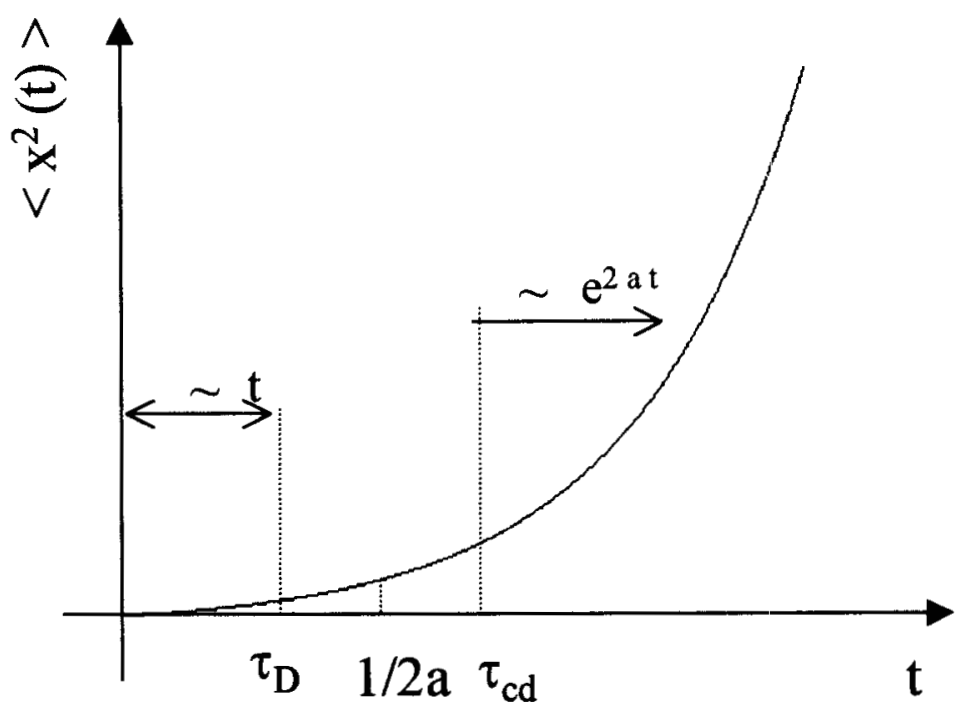

Fig. 1.4: Desplazamiento cuadrático medio para la variable $\mathrm{x}(\mathrm{t})$

y su segundo momento de la Ec. (1.14) está dado por

$$
\left\langle x^{2}(t)\right\rangle=\left\langle h^{2}(t)\right\rangle e^{2 a t},
$$

con ayuda de las Ecs. (1.13) y (1.15) se tiene

$$
\left\langle x^{2}(t)\right\rangle=\left[\frac{D}{a}\left(1-e^{-\frac{t}{\tau}}\right)\right] e^{\frac{t}{\tau}},
$$

tal que $\tau \equiv \frac{1}{2 a}$. Hagamos ahora el análisis de tiempos cortos y tiempos largos.

i) Tiempos Cortos $(t<<\tau)$

$$
\left\langle x^{2}(t)\right\rangle=2 D t .
$$

Este resultado es similar a las Ecs. (1.3) y (1.10) de las secciones anteriores correspondientes al régimen difusivo, esto es, la región donde las fluctuaciones estocásticas juegan un papel importante. Por lo tanto, en el régimen de tiempos cortos de acuerdo con (1.19), el sistema localizado en el estado inestable inicial, se encuentra sometido a la presencia de las fluctuaciones estocásticas responsables del decaimiento dinámico del sistema alrededor de dicho estado inestable.

ii) Tiempos Largos $(t>>\tau)$

$$
\left\langle x^{2}(t)\right\rangle=\frac{D}{a} e^{2 a t}
$$


En este régimen de tiempos, podemos observar que el término dominante es la exponencial $e^{2 a t}$ que nos describe la evolución del desplazamiento cuadrático medio más allá del régimen difusivo. Es en éste régimen de tiempos donde desarrollaremos nuestro trabajo de tesis, el cual es conocido como el régimen cuasi-determinista.

En la Fig. 1.4, mostramos el comportamiento del desplazamiento cuadrático medio del sistema inestable en las dos escalas de tiempo. Para tiempos cortos se muestra un coportamiento lineal de acuerdo con (1.19), el cual corresponde al régimen difusivo $\left(\tau_{D}\right)$; y para tiempos largos el DCM se encuentra dominado por el factor $e^{2 a t}$ de acuerdo con (1.20), el cual corresponde al régimen cuasi-determinista.

De los resultados obtenidos en esta sección, podemos ahora hacer las siguientes comparaciones. En la descripción de Langevin, véase la Fig. 1.2, en el régimen de tiempos cortos el DCM (1.9), muestra un comportamiento parabólico correspondiente al de una patícula libre, mientras que en el régimen de tiempos largos se observa un comportamiento lineal o difusivo. Lo anterior significa que en el régimen de tiempos cortos la partícula browniana aún no siente la presencia del medio (fluido), por lo que su comportamiento es prácticamente como la de una partícula libre; mientras que en el régimen de tiempos largos la partícula entra en contacto con el medio a través de sus constantes colisiones (que dan cuenta de las fluctuaciones) con las moléculas del fluido.

De acuerdo con nuestros resultados, véase la Fig. 1.4, en el régimen de tiempos cortos el DCM muestra un comportamiento lineal o difusivo, dado por (1.19); en tanto que a tiempos largos el comportamiento del DCM es dominado por el régimen cuasideterminista. Esto significa que a tiempos cortos o régimen difusivo, el sistema situado en el estado inestable inicial del potencial, se encuentra sometido a la presencia de las fluctuaciones responsables del decaimiento de dicho estado inestable, y para tiempos largos el comportamiento del sistema es prácticamente determinista de tal forma que el efecto de las fluctuaciones ya no juegan un papel importante. Por lo tanto podemos concluir que el proceso de decaimiento del estado inestable se inicia en el régimen difusivo.

\subsection{Objetivo Principal}

Hasta principios de la última decada de los noventa del siglo pasado, la caracterización dinámica de los procesos estocásticos transitorios de sistemas inestables formulada en ol 
contexto de la dinámica de Langevin, estaba descrita de forma tal que la fuerza sistemática lineal asociada era derivable de una función potencial $[52,45,53,4]$. Hace relativamente poco tiempo, este esquema de Langevin ha sido generalizado al estudio de sistemas inestables rotacionales, los cuales una vez que abandonan el estado inestable inicial por efecto de las fluctuaciones, describen trayectorias rotacionales prácticamente deterministas. En este nuevo esquema, la fuerza sistemática lineal asociada es en general no derivable de un potencial [28]-[31], [38]-[40].

Entre los diversos métodos que han sido propuestos para estudiar la dinámica estocástica transitoria de sistemas inestables derivables de una función potencial, se encuentra el método de las escalas de tiempo, donde el interés principal radica en caracterizar el proceso de decaimiento del estado inestable calculando el tiempo durante el cual el sistema alcanza por vez primera un valor de referencia preestablecido en el caso de sistemas lineales, o el valor estacionario que le corresponde en el caso de sistemas no lineales; una vez que dicho sistema abandona el estado inestable inicial por efecto de pequeñas fluctuaciones.

Entre las distintas teorías propuestas en el estudio de los tiempos de paso, queremos destacar el de la formulación cuasi-determinista propuesta por F. de Pascuale et. al. [43], pues ésta será la formulación que adoptaremos en esta tesis. Esta teoría se considera una buena aproximación para caracterizar los procesos de decaimiento de los sistemas inestables únicamente en el régimen lineal, ya que suministra la descripción física del mecanismo responsable del decaimiento del estado inestable; el cual consiste en asumir que las fluctuaciones (ruido interno) cambian la condición inicial alrededor del estado inestable e inducen el decaimiento dinámico del sistema. Después de este efecto, la evolución dinámica del sistema es prácticamente determinista. La formulación de TCD se realiza básicamente a partir de la aproximación lineal de una ecuación tipo Langevin que puede escribirse, para un conjunto de $n$ variables físicas independientes y en presencia de fuerza externa $\mathbf{F}_{e}$, de la siguiente manera

$$
\dot{\mathbf{x}}=a \mathbf{x}+N(r) \mathbf{x}+\mathbf{F}_{e}+\mathbf{z}(t)
$$

donde $a>0$, $\mathbf{x}$ el vector columna que representa a las variables físicas, $N(r)$ es una función escalar que da cuenta de las contribuciones no lineales del sistema, siendo $r$ el cuadrado de la norma del vector $\mathbf{x}$, es decir, $r \equiv \mathbf{x}^{T} \mathbf{x}$ con $\mathbf{x}^{T}$ su vector transpuesto. La 
fuerza sistemática lineal $\mathbf{F}=a \mathbf{x}$ de dicha ecuación es claramente derivable de una función potencial. El formalismo de TCD fue propuesto esencialmente para describir la dinámica lineal de (1.21), en este sentido hemos acordado en nombrar a dicha formulación como "Formulación Estándar de la Teoría Cuasi-Determinista" (FETCD). Este formalismo junto con los tiempos de paso, han sido aplicados esencialmente para caracterizar la dinámica transitoria de algunos sistemas Láser, así como la detección de señales ópticas débiles de dichos sistemas $[62,61,58,32,36]$.

Por otro lado, el estudio de la dinámica estocástica transitoria de los mismos sistemas inestables (1.21) a través de TRNL, ha sido también desarrollado en el marco teórico del formalismo estándar de TCD, y en el esquema de las ecuaciones de Fokker-Planck asociadas a la dinámica (1.21), como puede constatarse en las Refs. [24, 17, 45, 53]. Ambos esquemas presentan ciertas ventajas una con respecto a la otra, entre las que podemos mencionar están las siguientes: la relajación dinámica de los sistemas inestables inducidos por ruido de color gaussiano, es mucho más simple usando TCD que el formalismo de Fokker-Planck $[55,4]$. Otra ventaja de TCD es que éste puede caracterizar la relajación completa de sistemas inestables no lineales; mientras que con el formalismo de FokkerPlanck, la aplicación a sistemas inestables ha sido desarrollado únicamente en el régimen lineal $[53,44,17]$. El esquema de Fokker-Planck admite una descripción de la dinámica en términos de ruidos no gaussianos tales como: ruido dicotómico (señales telegráficas) y ruido de Poisson [21, 22]; mientras que TCD ha sido formulada únicamente en términos de ruido blanco y ruido de color gaussianos [23]-[27], [37]. El estudio de TCD en términos de ruidos no gaussianos aún no ha sido desarrollado.

La formulación estándar de TCD admite una generalización a sistemas inestables rotacionales, y nuestro objetivo principal en esta tesis consiste en estudiar el proceso de decaimiento de los sistemas inestables rotacionales, tanto en el régimen lineal a través de los tiempos de paso, así como en el régimen no lineal a través de los tiempos de relajación no lineales; en un contexto matricial completamente general.

La dinámica tipo Langevin rotacional en la que estamos interesados puede escribirse de la siguiente manera

$$
\dot{\mathbf{x}}=a \mathbf{x}+\mathbf{W} \mathbf{x}+N(r) \mathbf{x}+\mathbf{F}_{e}+\mathbf{z}(t)
$$

donde todas las cantidades ya han sido definidas anteriormente salvo el nuevo término 
$\mathbf{W x}$, siendo $\mathbf{W}$ una matriz real antisimétrica, tal que $\mathbf{W}^{T}=-\mathbf{W}$ con $\mathbf{W}^{T}$ su matriz transpuesta. El conjunto de variables $\mathbf{x}$ ya no son necesariamente independientes debido a la nueva contribución en la fuerza sistemática lineal, dada por $\mathbf{F}=a \mathbf{x}+\mathbf{W} \mathbf{x}$. En este sentido $\mathbf{F}_{c}=$ ax representa la parte conservativa y $\mathbf{F}_{n c}=\mathbf{W} \mathbf{x}$ la parte no conservativa, puesto que $\nabla \times \mathbf{F}_{c}=0$ y $\nabla \times \mathbf{F}_{n c} \neq 0$. Por lo tanto la fuerza sistemática lineal ya no es en general derivable de una función potencial, a menos que la matriz $\mathbf{W}$ sea cero. La matriz W da cuenta del acoplamiento entre las variables del vector $\mathbf{x}$ y por tanto de los efectos de rotación del sistema inestable. En el Apéndice A se muestra como caso particular que toda matriz antisimétrica en el espacio de tres dimensiones, determina un vector $\vec{\omega}$ tal que el producto matricial de la matriz antisimétrica es igual al producto vectorial del vector $\vec{\omega}$, que representa al vector velocidad angular, es decir, $\mathbf{W} \mathbf{x}=\vec{\omega} \times \mathbf{x}$. La caracterización dinámica de los sistemas inestables rotacionales (1.22) en este nuevo esquema es lo que podemos nombrar como "Formulación Matricial de la Teoría Cuasi-Determinista" (FMTCD).

La caracterización dinámica de los sistemas inestables rotacionales a través de la DTP y de los TRNL se realizará, al igual que en la formulación estándar, en términos de los momentos estadísticos de orden $l$ definidos por $\left\langle r^{l}(t)\right\rangle$, donde $r$ es nuevamente la norma al cuadrado del vecto $\mathbf{x}$ de (1.22). Se puede verificar que la solución de la parte lineal de (1.22) en términos de la variable $r$ es muy similar a la solución lineal de (1.21), y esto es debido principalmente a las propiedades de la matriz W. Como consecuencia de esta similitud se mostrará que en ausencia de fuerza externa, la distribución de los tiempos de paso y los tiempos de ralajación no lineales para caracterizar el decaimiento dinámico del sistema (1.22) son exactamente las mismas escalas de tiempo obtenidas para caracterizar la dinámica (1.21); mientras que en presencia de fuerza externa dichas escalas de tiempo son casi iguales y la mínima diferencia como se verá en el contenido de esta tesis, es sólo la renormalización de la amplitud de la fuerza externa debido a la contribución de los elementos de la matriz antisimétrica $\mathbf{W}$.

Ante esta situación se podría afirmar en cierta forma que, si bien el formalismo generalizado de TCD propuesto para caracterizar la dinámica (1.22) que por naturaleza es rotacional, conduce prácticamente a las mismas escalas de tiempo obtenidas en la descripción de la dinámica (1.21). Entonces parecería que el formalismo generalizado de TCD para describir a la dinámica (1.22) no aporta ninguna contribución novedosa y por 
lo tanto no es un método apropiado para dicha descripción. Sin embargo esto no es así, como se mostrará en seguida.

Antes de justificar el formalismo generalizado de TCD, hagamos las siguientes tres preguntas que consideramos son fundamentales en el desarrolo de esta tesis. La primera es, ¿porqué el formalismo generalizado de TCD funciona para describir a los sistemas rotacionales (1.22) y conduce prácticamente a los mismos resultados de las escalas de tiempo obtenidas para describir la dinámica (1.21)?; la segunda es ¿qué ocurre con los efectos de rotación provenientes de la matriz W? y por último ¿existirá alguna dinámica que provenga de (1.22) de tal forma que sea semejante a la dinámica (1.21) para poder entonces justificar el formalismo generalizado de TCD?

Las respuestas a estas preguntas las podemos obtener si en la Ec. (1.22) se realiza el siguiente cambio de variable $\mathbf{y}=\mathrm{e}^{-\mathbf{W}_{t}} \mathbf{x}$, de tal forma que la dinámica de Langevin en el nuevo espacio transformado será

$$
\dot{\mathbf{y}}=a \mathbf{y}+N(r) \mathbf{y}+\mathbf{F}_{e}^{R}+\mathbf{z}_{R}(t)
$$

donde $\mathbf{F}_{e}^{R}=\mathrm{e}^{-\mathbf{W} t} \mathbf{F}_{e}, \mathbf{z}_{R}(t)=\mathrm{e}^{-\mathbf{W} t} \mathbf{z}(t)$ y $N(r)$ es la misma función de (1.22) puesto que $r=\mathbf{x}^{T} \mathbf{x}=\mathbf{y}^{T} \mathbf{y}$, es decir la norma es invariante ante dicha transformación. Podemos observar que la transformación elimina a la matriz $\mathbf{W}$ de la fuerza sistemática lineal y asocia sus efectos a la presencia de las fuerzas externa y fluctuante. En este nuevo espacio, el conjunto de variables y están ahora desacoplados. Por lo tanto la dinámica (1.23) es muy semejante a la dinámica (1.21) pero no igual debido precisamente a las nuevas fuerzas $\mathbf{F}_{e}^{R} \mathrm{y} \mathbf{z}_{R}(t)$.

En base a lo anterior surge ahora la siguiente pregunta. ¿Cuál es el significado de la transformación realizada y por lo consiguiente de los términos $\mathrm{F}_{e}^{R}$ y $\mathbf{z}_{R}(t)$ ? La respuesta a esta pregunta se encuentra en el Apéndice $A$, donde se demuestra que el término $\mathrm{e}^{-\mathbf{W} t}$ que realiza la transformación del espacio $\mathbf{x}$ al espacio $\mathbf{y}$ es precisamente una rotación dependiente del tiempo, es decir, $\mathrm{e}^{-\mathbf{W} t}=\mathbf{R}(t)$ donde $\mathbf{R}(t)$ satisface las propiedades de una matriz de rotación. Por lo tanto, en el espacio transformado de coordenadas la dinámica (1.23) es matemáticamente semejante pero no igual a (1.21), y la diferencia substancial es que las trayectorias estocásticas de la dinámica (1.23) son rotacionales, debido al carácter rotacional de las fuerzas externa $\mathrm{F}_{e}^{R}$ y fluctuante $\mathbf{z}_{R}(t)$. Es precisamente en este nuevo espacio transformado de coordenadas, donde podemos entender porqué en 
ausencia de fuerza externa, las escalas de tiempo obtenidas para describir la dinámica (1.22) son exactamente las mismas que las que se obtienen para describir la dinámica (1.21); en tanto que en presencia de fuerza externa, las escalas de tiempo son casi iguales y la diferencia es la renormalización de la amplitud de la fuerza externa. Esta situación se mostrará con más detalle en el contenido de esta tesis cuando estudiemos a los sistemas rotacionales de dos y tres variables.

El contenido de esta tesis está estructurado de la siguiente manera:

En el Cap. 2, se estudia la caracterización dinámica de los sistemas inestables (1.21), tanto en ausencia como en presencia de fueza externa, a través de los tiempos de paso y de los tiempos de relajación no lineales, mediante el formalismo de TCD en el contexto de ruidos gaussianos en general. En ausencia de fuerza externa obtenemos resultados explícitos para los casos de ruido blanco y ruido de color gaussianos; y estudiamos el Modelo de Landau lineal y no lineal en el caso de dos variables a través de TP y de TRNL. En presencia de fuerza externa constante estudiamos los sistemas inestables de dos y tres variables, únicamente con ruido blanco gaussiano.

En el Cap. 3, proponemos el formalismo generalizado de TCD para caracterizar a los sistemas inestables rotacionales de la forma (1.22) en ausencia de la fuerza externa $\mathbf{F}_{e}, \mathrm{y}$ en un contexto teórico matricial completamente general. Estudiamos la caracterización dinámica de dichos sistemas en presencia de ruido blanco y ruido de color gaussianos; y demostramos que en el caso de ruido blanco la descripción se realiza en general para cualquier número de variables físicas, en tanto que en el caso de ruido de color, el formalismo de TCD requiere del número específico de variables y de ciertas aproximaciones para satisfacer las condiciones de dicho formalismo. Para este propósito estudiaremos a los sistemas rotacionales de dos y tres variables en presencia de ruido de color gaussiano. Como consecuencia de nuestro estudio se muestra que tanto en el caso de ruido blanco como en el de ruido de color los resultados de las escalas de tiempo son los mismos que los obtenidos en el Cap. 2. La respuesta de esta igualdad en los resultados se da con detalle en la Sec. 3.4, donde discutimos la equivalencia entre las dinámicas de Langevin en el espacio $\mathbf{x}$ y en el espacio transformado $\mathbf{y}$, así como la semejanza de la dinámica de este espacio transformado con la dinámica (1.21). Aunque el estudio teórico se realiza para cualquier número $n$ de variables, los cálculos de la simulación numérica, se darán sólo para los sistemas lineales de dos variables, sin pérdida en la generalidad de los resultados. 
En este caso particular se demuestra la semejanza que existe entre la dinámica lineal de (1.23) con la dinámica lineal de (1.21), ya que en ambos casos las trayectorias estocásticas correspondientes son líneas rectas, sólo que el espacio transformado de coordenadas y las líneas rectas están rotadas de manera aleatoria alrededor del origen de coordenas del plano $\left(y_{1}, y_{2}\right)$, como se puede ver en las Figs. 3.3 y 3.4

En el Cap. 4 se describe, en el espacio transformado de coordenadas $\mathbf{y}$, el formalismo generalizado de TCD para caracterizar el decaimiento dinámico de los sistemas inestables rotacionales inducidos por ruido blanco gaussiano y en presencia de fuerza externa. En la Sec. 4.2, se estudia la caracterización dinámica de los sistemas rotacionales de dos variables en presencia de fuerza externa constante. Se mostrará que las escalas de tiempo comparadas con las obtenidas en la Sec. 2.5.1 son muy parecidas aunque no exactamente iguales, ya que en este caso la amplitud de la fuerza externa es renormalizada debido a la contribición de la matriz W. Sin embargo, mostraremos que esta contribución es prácticamente insignificante en las escalas de tiempo que caracterizan al sistema. La presencia de la fuerza externa introduce dos regímenes de validéz de las escalas de tiempo. Uno corresponde a la situación en la que la amplitud de la fuerza externa es mayor que la intensidad del ruido interno y el otro, corresponde a la aproximación de fuerza débil, es decir, cuando la amplitud de dicha fuerza es menor o igual que la intensidad del ruido interno. En ambos casos, se desarrollan las aproximaciones de las escalas de tiempo y se demuestra que la teoría de TCD es válida en el régimen de aproximación débil de la fuerza externa por las siguientes razones: de acuerdo con los resultados de la simulación numérica, mostraremos que si la amplitud de la fuerza externa es mayor que la del ruido interno, entonces para tiempos largos de observación, las trayectorias estocásticas generadas por la dinámica lineal de (1.23) son trayectorias rotacionales en forma de "bucles" que emergen del origen de coordenadas del plano $\left(y_{1}, y_{2}\right)$, como se puede constatar en la Fig. 4.1. Para esta región de aproximación, la teoría no describe de manera precisa los efectos de rotación del sistema, como se puede verificar en la Fig. 4.2.

Sin embargo, en la misma escala de tiempos largos, si la fuerza es débil lo que podemos observar en los resultados de la simulación numérica, es que las trayectorias estocásticas anteriores son casi líneas rectas, es decir, los efectos de rotación o "bucless" son prácticamente indistinguibles. En esta región de aproximación la teoría describe de manera precisa el proceso de decaimiento del sistema, ver la Fig. 4.3 
En el caso de fuerza externa dependiente del tiempo, estudiamos sólo a los sistemas de dos variables y suponemos que dicha fuerza es una cantidad que fluctúa en el tiempo a través de su fase. En este caso los requerimientos de TCD se satisfacen si además de la aproximación de fuerza débil, suponemos que la intensidad de las fluctuaciones de fase sean pequeñas.

La formulación teórica anterior ha sido aplicada al estudio de la dinámica transitoria de los sistemas Láser para detectar señales ópticas débiles a través de TRNL y de la DTP. En particular en la Sec. 4.3.2 se estudia la deteccción de las señales ópticas débiles mediante el receptor de salida, a través de TRNL. En la Sec. 4.3.3, estudiamos el sistema Láser de la sección anterior en presencia de campo externo dependiente del tiempo, mediante la distribución de los tiempos de paso y comparamos con los resultados de la Ref. [9].

Por último en el caso de fuerza externa constante, estudiamos a los sistemas rotacionales de tres variables y mostramos la similitud que existe con la dinámica de los sistemas rotacionales de dos variables. La descripción teórica es consistente nuevamente en la región de fuerza externa débil, de acuerdo con los resulatdos de la simulación numérica. Finalmente, en el Cap. 5 damos las conclusiones y perspectivas de esta tesis.

Nuestro estudio se complementa con ayuda de algunos Apéndices que sustentan el contenido de esta tesis.

En el Apéndice A presentamos algunas de las propiedades inherentes a la matriz antisimétrica $\mathbf{W}$, y la demostración general de que la transformación $\mathrm{e}^{-\mathbf{W} t}$ es una matriz de rotación. En el Apéndice $B$, damos los fundamentos de la dinámica de Langevin descrita por Fox y Uhlenbeck [12]. Por último en el Apéndice $\mathrm{C}$, se dan los cálculos explícitos que justifican los resultados analíticos de los Caps. 2 y 4. 


\section{Capítulo 2}

\section{Caracterización dinámica de sistemas inestables. Formulación Estándar}

\subsection{Definición general de estados inestables}

Como hemos mencionado anteriormente, nuestro interés en este trabajo radica en caracterizar el decaimiento dinámico de sistemas inestables a través de la formulación cuasideterminista; y para ello será necesario introducir la definición general de estados inestables a partir de un análisis puramente determinista.

Hagamos el análisis para el caso de una variable física $x$ cualesquiera y supongamos que su evolución dinámica satisface la ecuación lineal $\dot{x}=a x$ tal que $a>0$. En este caso la fuerza sistemática lineal es $f(x)=a x$, por lo que el potencial asociado a dicha fuerza es $V(x)=-\frac{1}{2} a x^{2}$, que corresponde al potencial de la Fig. 1.3.

Para un conjunto de $n$-variables físicas $x_{i}$ independientes, con $i=1, \ldots, n$, tal que la evolución dinámica de cada variable puede escribirse en forma vectorial como $\dot{\mathbf{x}}=a \mathbf{x}$ con $\mathrm{x}$ el vector columna de dichas variables. Nuestro interés está en formular la dinámica determinista en términos de una variable definida como $r$ que representa la norma al cuadrado del vector $\mathbf{x}$, es decir $r \equiv x^{2}=\mathbf{x}^{T} \mathbf{x}=x_{1}^{2}+\cdots+x_{n}^{2}$. En términos de la nueva variable $r$ la ecuación dinámica anterior será $\dot{r}=2 a r ;$ y en este caso la fuerza sistemática es $f(r)=2 a r$ y su correspondiente potencial es ahora $V(r)=-a r^{2}$, que representa a una parábola invertida en el espacio de $r$.

De acuerdo con lo anterior, podemos ahora establecer que la definición general de estados inestables no lineales para un conjunto de $n$-variables físicas, se puede escribirse 
de la siguiente manera

$$
\dot{r}=f(r)=\frac{r\left(r_{s t}-r\right)}{C_{0}+r g(r)},
$$

donde la constante $C_{0}=\frac{r_{s t}}{2 a}$ y $g(r)$ es un polinómio de la forma $\sum_{k=0}^{m} g_{k} r^{k}$. Podemos observar de (2.1) que $f(r)$ tiene dos raices, una raíz está en $r=0$ que corresponde al estado inestable del potencial asociado a $f(r)$, es decir $f(r)=-\frac{d V(r)}{d r}$; por lo tanto $\left.f^{\prime}(r)\right|_{r=0}>0$. La otra raíz es para $r=r_{s t}$ el cual corresponde al estado estable del potencial asociado a $f(r)$, por lo que $\left.f^{\prime}(r)\right|_{r=r_{s t}}<0$. Es conveniente resaltar que el caso lineal de (2.1) corresponde a $\dot{r}=2 a r$, lo cual se obtiene despreciando términos de orden superior en $r$.

\subsection{Análisis cualitativo de la teoría cuasi-determi- nista}

La teoría cuasi-determinista desarrollada por De Pascuale et. al. [43] para describir la dinámica estocástica transitoria es una buena descripción del problema, puesto que suministra el mecanismo preciso responsable del decaimiento del sistema alrededor de su estado inestable. La teoría establece que dicho mecanismo no es otra cosa que la fluctuación de la condición inicial del sistema alrededor de su estado inestable. Una vez que el sistema abandona dicho estado inestable, su evolución es prácticamente determinista.

Hagamos una descripción cualitativa de lo anterior tomando únicamente el régimen lineal de la dinámica $(2.1)$, es decir

$$
\dot{r}=2 a r
$$

con $a>0$.La solución de esta ecuación es

$$
r(t)=r(0) \mathrm{e}^{2 a t}
$$

De esta ecuación podemos notar que en $t=0$, el sistema tiene el valor inicial $r(0)$ y por lo tanto se encuentra localizado en el punto inestable $(0, \ldots, 0)$ del potencial $V(r)=-a r^{2}$ asociado al proceso (2.2). En el caso de tres dimensiones el potencial correspondería a un paraboloide de revolución invertido en la dirección $-z$ cuyo vértice coincide con el origen de coordenadas $x y z$. Por lo tanto desde el punto de vista clásico $r(0)=0$, lo cual significa que el sistema permanecerá en el estado inestable del potencial por tiempo indefinido. 
sin que ocurra ningún decaimiento dinámico del sistema a menos que exista algún agente externo que saque al sistema de dicho estado.

La hipótesis de TCD es asumir que la condición inicial es una cantidad fluctuante, es decir suponemos que la condición inicial $r(0)$ fluctúa alrededor del estado inestable; ésta fluctuación (de cualquier tipo) será suficiente para sacar al sistema de su estado inestable inicial e inducir su correspondiente decaimiento dinámico. En este sentido la condición inicial $r(0)$ toma ahora un conjunto de valores aleatorios $h$, tal que $r(0)=h^{2}$. Así la solución (2.3) adquiere el carácter de un proceso cuasi-determinista dado por

$$
r(t)=h^{2} \mathrm{e}^{2 a t} .
$$

Una vez que, el sistema abandona el estado inestable por efecto de las fluctuaciones iniciales, éste evolucionará indefinidamente para todo tiempo $t>0$ siguiendo prácticamente su trayectoria determinsta. De (2.4) podemos ver claramente que existe un conjunto de tiempos aleatorios $t_{i}$ para los cuales el proceso $r(t)$ alcanza un valor predeterminado $R^{2}$, tal que $r\left(t_{i}\right) \equiv R^{2}$; en este caso el promedio de $t_{i}$ será

$$
<t_{i}>=\frac{1}{2 a}\left\langle\ln \left(\frac{R^{2}}{h^{2}}\right)\right\rangle,
$$

que corresponde a la escala de tiempo universal, característica del decaimiento de estados inestables. La justificación de la hipótesis de la fluctuación de la condición inicial, así como la estadística de la variable $h$ será obtenida mediante el análisis formal de TCD, que daremos a continuación.

\subsection{Formulación Estándar de la teoría cuasi-deter- minista}

\subsubsection{Efecto de condiciones iniciales distribuidas}

El formalismo de TCD se describe en el régimen lineal de una ecuación tipo Langevin no lineal con ruido aditivo, el cual puede escribirse para un conjunto de $n$ variables físicas independientes de la siguiente forma

$$
\dot{\mathbf{x}}=a \mathbf{x}+N(r) \mathbf{x}+\mathbf{z}(t) .
$$


El primer término es la fuerza sistemática lineal. El segundo término da cuenta de las contribuciones no lineales del sistema, siendo $N(r)$ una función escalar de $r$. El tercer término se refiere a la fuerza fluctuante o ruido con elementos $\xi_{i}(t)$, cuyas propiedades estadísticas deberán ser asignadas según sea el caso. En este trabajo supondremos que $\mathrm{z}(t)$ es un proceso gaussiano por lo tanto únicamente requerimos de sus dos primeros momentos, los cuales supondremos que su valor medio es nulo y su función de correlación satisface la siguiente propiedad

$$
<\xi_{i}(t) \xi_{j}\left(t^{\prime}\right)>=\lambda \delta_{i j} \gamma\left(\left|t-t^{\prime}\right|\right), \quad i, j=1, \ldots, n
$$

donde $\lambda$ es una constante que mide la intensidad de la fluctuación, $\delta_{i j}$ es la delta de Kronecker. Suponer que $\gamma$ depende de la diferencia de tiempos significa que es un proceso estacionario.

El análisis de TCD inicia con la approximación lineal de (2.6), es decir

$$
\dot{\mathbf{x}}=a \mathbf{x}+\mathbf{z}(t)
$$

cuya solución puede escribirse como

$$
\mathbf{x}(t)=\mathrm{e}^{a t} \mathbf{h}(t)
$$

donde $\mathbf{h}(t)$ está dada por

$$
\mathbf{h}(t)=\mathbf{x}(0)+\mathbf{g}(t), \quad \mathbf{g}(t) \equiv \int_{0}^{t} \mathrm{e}^{-a s} \mathbf{z}(s) d s .
$$

El primer término se refiere a la condición inicial del sistema la cual puede ser fija o estar distribuida alrededor del cero.

La escala de tiempo cuasi-determinista (1.20) nos dice que para tiempos largos, el proceso $\mathbf{h}(t)$ debe ser una constante y por tanto juega el papel de una condición inicial efectiva. Esto es así ya que para valores pequeños de cada elemento del vecto $\mathbf{z}(t)$ el proceso $\mathbf{h}(t)$ satisface que

$$
\lim _{t \rightarrow \infty} \frac{d \mathbf{h}(t)}{d t}=\lim _{t \rightarrow \infty} e^{-a t} \mathbf{z}(t) \rightarrow 0
$$

por lo tanto $\mathbf{h}(\infty)=\mathbf{h}$ donde $\mathbf{h}$ es un vector constante formado por $h_{i}=x_{0 i}+g_{i}$ Variables Aleatorias Gaussianas (VAG), puesto que cada elemento de $\xi_{i}(t)$ es una VAG. 
En este límite de tiempos largos el proceso (2.9) es un proceso cuasi-determinista, y que en términos de la variable $r$ se puede escribir como

$$
r(t)=\mathbf{x}^{T} \mathbf{x}=h^{2} \mathrm{e}^{2 a t},
$$

donde $h^{2} \equiv \mathbf{h}^{T} \mathbf{h}=h_{1}^{2}+\cdots+h_{n}^{2}$; con lo que la Ec. (2.12) es consistente con la hipótesis de la condición inicial (2.4). La distribución de tiempos de paso para alcanzar el valor de referencia $R^{2}$ es entonces

$$
<t_{i}>=\frac{1}{2 a}\left\langle\ln \left(\frac{R^{2}}{h^{2}}\right)\right\rangle .
$$

El tiempo de paso (2.13) debe calcularse ahora conociendo la estadística de la variable $h$ a través de la densidad de probabilidad $P(h)$, que a su vez requiere del conocimiento de la densidad de probabilidad conjunta $P\left(h_{1}, \ldots, h_{n}\right)$.

Para poder llevar a cabo el cálculo de $P\left(h_{1}, \ldots, h_{n}\right)$ se requiere de las propiedades de la matriz $\sigma_{i j}$ definida por

$$
\sigma_{i j} \equiv<h_{i} h_{j}>-<h_{i}><h_{j}>=\sigma_{i j}^{0}+\sigma_{i j}^{R}+\sigma_{i j}^{S R}
$$

donde

$$
\begin{gathered}
\sigma_{i j}^{0} \equiv<x_{i}(0) x_{j}(0)>-<x_{i}(0)><x_{j}(0)>, \\
\sigma_{i j}^{R} \equiv<g_{i} g_{j}>-<g_{i}><g_{j}> \\
\sigma_{i j}^{S R} \equiv<x_{0 i} g_{j}>-<x_{0 i}><g_{j}>.
\end{gathered}
$$

La matriz $\sigma_{i j}^{0}$ da cuenta de los efectos de las condiciónes iniciales, la matriz $\sigma_{i j}^{R}$ toma en cuenta el efecto de las fluctuaciones causadas por el ruido y la matriz $\sigma_{i j}^{S R}$ muestra justamente el acoplamiento del sistema en su estado inicial con el ruido.

Como se puede ver de (2.14), para poder calcular $\sigma_{i j}$ se requiere de sus dos primeros momentos $<h_{i}>\mathrm{y}<h_{i} h_{j}>$. De acuerdo con (2.10) se tiene que $<h_{i}>=<x_{i}(0)>\mathrm{y}$ para el segundo momento tenemos

$$
<h_{i}(t) h_{j}(t)>=K_{0}+K_{R}(t)+K_{S R}(t)
$$

tal que

$$
K_{0} \equiv<x_{\imath}(0) x_{j}(0)>
$$




$$
\begin{gathered}
K_{R}(t) \equiv<g_{i}(t) g_{j}(t)>=\int_{0}^{t} \int_{0}^{t} \mathrm{e}^{-a\left(s+s^{\prime}\right)}<\xi_{i}(s) \xi_{j}\left(s^{\prime}\right)>d s d s^{\prime} \\
K_{S R}(t) \equiv 2 \int_{0}^{t} \mathrm{e}^{-a s}<x_{i}(0) \xi_{j}(s)>d s
\end{gathered}
$$

Para poder evaluar el primer y el tercer término vamos a recurrir del siguiente argumento. Consideremos que al tiempo $t=0$ el sistema esté localizado en algún estado estacionario estable. Esto se logra a través de la definición matemática de la evolución del sistema para tiempos $t<0$, desde un estado arbitrario en $t=-\infty$. Por tanto la preparación de las condiciones iniciales del sistema en $t=0$, será el estado estacionario de la siguiente ecuación lineal

$$
\dot{\mathbf{x}}_{0}=-a_{0} \mathbf{x}_{0}+\mathbf{z}(t)
$$

donde $a_{0}>0$ y $\mathbf{z}(t)$ satisface las mismas propiedades dadas anteriormente excepto que la intensidad del ruido puede ser en general diferente de $\lambda$, digamos $\lambda^{\prime}$. Por simplicidad consideraremos que $\lambda=\lambda^{\prime}$. La solución de (2.18) está dada por

$$
\mathbf{x}(0)=\int_{-\infty}^{0} \mathrm{e}^{a_{0} s} \mathbf{z}(s) d s .
$$

La dinámica (2.18) describe la situación física en la que al tiempo $t=0$ el sistema sufre un cambio brusco en el parámetro de control, de $-a_{0} \rightarrow a$, el cual deja al sistema inicialmente localizado alrededor de un estado inestable.

De la expresión (2.19) se obtiene que $\left\langle x_{i}(0)\right\rangle=0$ y su correlación $K_{0}$ viene dada por

$$
K_{0}=\lambda \Lambda_{0} \delta_{i j}
$$

con $\Lambda_{0}$ definido por

$$
\Lambda_{0} \equiv \int_{-\infty}^{0} \int_{-\infty}^{0} \mathrm{e}^{a_{0}\left(s+s^{\prime}\right)} \gamma\left(\left|s-s^{\prime}\right|\right) d s d s^{\prime}
$$

Por lo tanto la matriz de las condiciones iniciales $\sigma_{i j}^{0}=\lambda \Lambda_{0} \delta_{i j}$ es diagonal, con elementos $\sigma_{i i}^{0} \equiv \sigma_{0}^{2}=\lambda \Lambda_{0}$. Entonces el conjunto de variables $x_{i}(0)$ están descorrelacionadas para $i \neq j$.

Con estos resultados tenemos que la densidad de probabilidad conjunta está dada por

$$
P_{s t}\left(x_{0_{1}}, \ldots, x_{0 n}\right)=\text { cte } \mathrm{e}^{-\alpha^{2}\left(x_{0_{1}}^{2}+\cdots+x_{0 n}^{2}\right)} .
$$


Bajo éstas condiciones se tiene que la correlación (2.16) se puede escribir de la siguiente forma

$$
<h_{i}(t) h_{j}(t)>=\lambda\left[\Lambda_{0}+\Lambda_{R}(t)+\Lambda_{S R}(t)\right] \delta_{i j}
$$

donde

$$
\begin{aligned}
\Lambda_{R}(t) & \equiv \int_{0}^{t} \int_{0}^{t} \mathrm{e}^{-a\left(s+s^{\prime}\right)} \gamma\left(\left|s-s^{\prime}\right|\right) d s d s^{\prime}, \\
\Lambda_{S R}(t) & \equiv \int_{0}^{t} \int_{-\infty}^{0} \mathrm{e}^{-a s+a_{0} s^{\prime}} \gamma\left(\left|s-s^{\prime}\right|\right) d s d s^{\prime} .
\end{aligned}
$$

De las expresiones anteriores podemos mostrar que en el límite de tiempos largos, tales cantidades tienden a ser constantes, es decir $\Lambda_{R}(\infty)=\Lambda_{R}$ y $\Lambda_{S R}(\infty)=\Lambda_{S R}$, y de acuerdo con (2.19) se tiene que $\left\langle h_{i}(t)\right\rangle=0$. De estos resultados tenemos que la matriz $\sigma_{i j}^{R}=$ $\lambda \Lambda_{R} \delta_{i j}$ es diagonal, cuyos elementos son $\sigma_{i i}^{R} \equiv \sigma_{R}^{2}=\lambda \Lambda_{R}$; también la matriz $\sigma_{i j}^{S R}=$ $\lambda \Lambda_{S R} \delta_{i j}$ es diagonal, tal que $\sigma_{i i}^{S R} \equiv \sigma_{S R}^{2}=\lambda \Lambda_{S R}$. Por lo tanto la matriz $\sigma_{i j}=\lambda\left(\Lambda_{0}+\right.$ $\left.\Lambda_{R}+\Lambda_{S R}\right) \delta_{i j}$ es diagonal con elementos $\sigma_{i i}=\sigma_{0}^{2}+\sigma_{R}^{2}+\sigma_{S R}^{2} \equiv \sigma^{2}$ e igual a

$$
\sigma^{2}=\lambda\left(\Lambda_{0}+\Lambda_{R}+\Lambda_{S R}\right) \text {. }
$$

Esto nos dice que el conjunto de variables aleatorias $h_{i}$ están descorrelacionadas para $i \neq j$ y por tanto son variables aleatorias independientes.

Bajo estas circunstancias la densidad de probabilidad conjunta será

$$
P\left(h_{1}, \ldots, h_{n}\right)=\text { cte } \mathrm{e}^{-\alpha^{2}\left(h_{1}^{2}+\cdots+h_{n}^{2}\right)},
$$

tal que $\alpha^{2} \equiv \frac{1}{2 \sigma^{2}}$.

La densidad de probabilidad marginal $P(h)$ podrá ahora calcularse a partir de (2.27) mediante la transformación del espacio de variables independientes $\mathbf{h}=\left(h_{1}, \ldots, h_{n}\right)$ al espacio de variables independientes $\mathbf{u}=\left(h, u_{2}, \ldots, u_{n}\right)$, es decir

$$
P\left(h_{1}, \ldots, h_{n}\right) d h_{1} \cdots d h_{n} \rightarrow P\left(h, u_{2}, \ldots, u_{n}\right) d V
$$

donde $d V=J(\mathbf{u}) d h \cdot d u_{2} \cdots d u_{n}$ es el jacobiano de la transformación, dado por

$$
J(\mathbf{u})=\left|\begin{array}{ccc}
\frac{\partial h_{1}}{\partial h} & \ldots & \frac{\partial h_{1}}{\partial u_{n}} \\
\vdots & \ddots & \vdots \\
\frac{\partial h_{n}}{\partial h} & \ldots & \frac{\partial h_{n}}{\partial u_{n}}
\end{array}\right| .
$$


En nuestro caso, la región $h \leq \sqrt{h_{1}^{2}+\ldots+h_{n}^{2}} \leq h+d h$ es un cascarón hiperesférico de radio interno $h$ y radio externo $h+d h$, tal que $d V=$ cte $h^{n-1} d h$. Finalmente, la densidad de probabilidad marginal para el módulo $h$ tiene la siguiente expresión

$$
P(h)=\frac{2 \alpha^{n}}{\Gamma\left(\frac{n}{2}\right)} h^{n-1} \mathrm{e}^{-\alpha^{2} h^{2}} .
$$

De forma enteramente análoga podemos obtener la densidad de probalilidad $P\left(x_{0}\right)$, donde definimos $x_{0}^{2} \equiv \mathbf{x}_{0}^{T} \mathbf{x}_{0}=x_{1}^{2}(0)+\cdots+x_{n}^{2}(0)$. Por lo tanto

$$
P_{s t}\left(x_{0}\right)=\frac{2 \alpha_{0}^{n}}{\Gamma\left(\frac{n}{2}\right)} x_{0}^{n-1} \mathrm{e}^{-\alpha_{0}^{2} x_{0}^{2}}
$$

Finalmente la escala de tiempo (2.13) en el límite de ruido pequeño, tiene la siguiente estructura

$$
<t_{i}>=\frac{1}{2 a}\left\{\ln \left(\frac{R^{2}}{2 \sigma^{2}}\right)-\psi\left(\frac{n}{2}\right)\right\}
$$

donde la forma explícita de $\sigma^{2}$ dependerá de las condiciones iniciales ya sean fijas o distribuidas. Esta escala de tiempo se conoce en la literatura como La Distribución de Tiempos de Paso (DTP).

\subsubsection{Efecto de condiciones iniciales fijas}

Si la condición inicial del sistema es fija, es decir $\mathbf{x}(0)=0$, lo cual quiere decir que el sistema está localizado en el origen de coordenadas o en el estado inestable del potencial. En el lenguaje de probabilidad, esto quiere decir que $P(\mathbf{x})=\delta\left(\mathbf{x}-\mathbf{x}_{0}\right)$, por lo tanto la probabilidad de encontrar al sistema en el origen $\mathbf{x}_{0}=(0, \ldots, 0)$ es la unidad. Lo anterior nos dice desde el punto de vista experimental que la anchura o incertidumbre de la distribución inicial es más pequeña que la intensidad de las fluctuaciones que ocasionan el decaimiento. Sin embargo en muchas situaciones experimentales, ambas cantidades serán originadas por una única fuente de ruido, así que ellas pueden ser del mismo orden de magnitud como hemos supuesto anteriormente. Para condiciones iniciales fijas la varianza será igual a $\sigma^{2}=\lambda \Lambda_{R}$ y la escala de tiempo es la misma que (2.32). 


\subsubsection{Tiempo de Paso inducidos por ruido blanco gaussiano}

Vamos ahora a considerar el caso en que la función de correlación (2.7) satisface la condición

$$
<\xi_{i}(t) \xi_{j}\left(t^{\prime}\right)>=2 D \delta_{i j} \delta\left(t-t^{\prime}\right)
$$

con lo que la fuerza fluctuante satisface las propiedades de Ruido Blanco Gaussiano (RBG). De acuerdo con esto, podemos verificar que la constante $\lambda=2 D$ y $\gamma\left(\left|t-t^{\prime}\right|\right)=$ $\delta\left(t-t^{\prime}\right)$. En este caso las cantidades involucradas en (2.23) serán

$$
\Lambda_{0}=\frac{1}{a_{0}}, \quad \Lambda_{R}(t)=\frac{1}{a}\left(1-\mathrm{e}^{-2 a t}\right), \quad \Lambda_{S R}(t)=0 .
$$

Por lo tanto en el límite de tiempos largos la expresión para la varianza (2.26) será

$$
\sigma^{2}=\left(\frac{1}{a_{0}}+\frac{1}{a}\right) D
$$

En estas circunstancias el tiempo de paso (2.32) se reduce a

$$
<t_{i}>=\frac{1}{2 a}\left\{\ln \left[\frac{a_{0} a R^{2}}{2 D\left(a_{0}+a\right)}\right]-\psi\left(\frac{n}{2}\right)\right\} .
$$

Si las condiciones iniciales son fijas, entonces $\sigma^{2}=\frac{D}{a}$.

\subsubsection{Tiempo de Paso inducidos por ruido de color gaussiano}

El estudio correspondiente con ruido de color es un caso menos ideal, puesto que debemos tomar en cuenta el caracter no-markoviano del problema a través del tiempo de correlación del ruido [24]. Veremos también que el problema con ruido de color, introduce de manera natural el acoplamiento del estado inicial del sistema al tiempo $t=0$ con el ruido $\mathbf{z}(t)$, es decir que las cantidades $\Lambda_{0}$ y $\Lambda_{S R}(t)$ serán distintas de cero, pues en general $<x_{i}^{2}(0)>\mathrm{y}$ la correlación $<x_{i}(0) \xi_{i}(t)>$ también lo son.

Si $\mathbf{z}(t)$ satisface las propiedades de ruido de color, entonces la función de correlación (2.7) es ahora

$$
<\xi_{i}(t) \xi_{j}\left(t^{\prime}\right)>=\frac{D}{\tau} \delta_{i j} \mathrm{e}^{-\frac{\left|t-t^{\prime}\right|}{\tau}},
$$

donde $\tau$ es el tiempo de correlación. Para este caso la constante $\lambda=\frac{D}{\tau}$ y la función $\gamma\left(\left|t-t^{\prime}\right|\right)=\mathrm{e}^{-\frac{\left|t-t^{\prime}\right|}{\tau}}$. 
El problema con ruido de color sugiere tres casos posibles a analizar. El primero es el caso más simple en el que la condición inicial $\mathbf{x}(0)$ está fija en el estado inestable, de modo que $\mathbf{x}(0)=0$ y por lo tanto $\left\langle x_{i}(0) \xi_{i}(t)\right\rangle=0$. El segundo caso sería cuando la condición inicial $\mathbf{x}(0)$ esté distribuida alrededor del estado inestable, pero desacoplado del ruido, de modo que también $\left\langle x_{i}(0) \xi_{i}(t)\right\rangle=0$. Esto significa que la fuente de ruido para la dinámica a timpos $t<0$ no es necesariamente la misma que para tiempos $t>0$, de manera que el sistema puede iniciar desde un punto distribuido aleatoriamente. La tercera situación exhibe un estudio más interesante, pues en éste caso las condiciones iniciales se consideran distintas de cero y además acopladas con el ruido, es decir que $<x_{i}(0) \xi_{i}(t)>\neq 0$ y por tanto $\Lambda_{0}$ y $\Lambda_{S R}(t)$ son diferentes de cero.

(i) Condiciones iniciales fijas

Para este caso, el único término distinto de cero en (2.23) es $\Lambda_{R}(t)$, por lo que después de cierta algebra se puede mostrar que

$$
<h_{i}^{2}(t)>=\Lambda_{R}(t)=\frac{D}{a(1+a \tau)}\left[1-\mathrm{e}^{-2 a t}+\frac{2 a \tau}{(1-a \tau)}\left(\mathrm{e}^{-\left(a+\tau^{-1}\right) t}-\mathrm{e}^{-2 a t}\right)\right],
$$

el cual ha sido escrito de esta manera ya que en el límite de $\tau$ pequeño, éste termino coincide con el de ruido blanco. En el límite de tiempos largos tales que $2 a t>>1$, la varianza (2.26) se reduce a

$$
\sigma^{2}=\frac{D}{a(1+a \tau)}
$$

con lo que podemos concluir que el efecto del ruido de color con respecto al ruido blanco es una renormalización de la intensidad del ruido por el factor $1 /(1+a \tau)$.

(ii) Condiciones iniciales distribuidas. Caso desacoplado

Aquí supondremos que $\left\langle x_{i}(0) \xi_{i}(t)>=0\right.$ pero $\left\langle x_{i}^{2}(0)>\neq 0\right.$ y por lo tanto de acuerdo con (2.23) tenemos que

$$
<h_{i}^{2}(t)>=\frac{D}{a_{0}\left(1+a_{0} \tau\right)}+\Lambda_{R}(t) .
$$

En el límite de tiempos largos obtenemos que

$$
\sigma^{2}=\frac{D}{a_{0}\left(1+a_{0} \tau\right)}+\frac{D}{a(1+a \tau)}
$$

(iii) Condiciones iniciales distribuidas. Caso acoplado 
En este caso requerimos de los tres términos $\Lambda_{0}, \Lambda_{R}(t)$ y $\Lambda_{S R}(t)$ de (2.23) donde se puede mostrar que

$$
\Lambda_{S R}(t)=\frac{2 D \tau}{\left(1+a_{0} \tau\right)(1+a \tau)}\left[1-\mathrm{e}^{-\left(a+\tau^{-1}\right) t}\right]
$$

Con esto podemos mostrar que la varianza está dada por

$$
\sigma^{2}=\frac{D}{a_{0}\left(1+a_{0} \tau\right)}+\frac{D}{a(1+a \tau)}+\frac{2 D \tau}{(1+a \tau)\left(1+a_{0} \tau\right)}
$$

De acuerdo con los tres casos anteriores, se puede verificar que en el límite de $\tau$ pequeño los tiempos de paso se pueden reducir a las siguientes expresiones.

\section{(i) Condiciones iniciales fijas}

$$
<t_{i}>_{f}=<t_{i}(\tau=0)>_{f}+\frac{\tau}{2}+\mathcal{O}\left(\tau^{2}, D\right),
$$

donde

$$
<t_{i}(\tau=0)>_{f}=\frac{1}{2 a}\left\{\ln \left[\frac{a R^{2}}{2 D}\right]+\psi\left(\frac{n}{2}\right)\right\},
$$

es la escala de tiempo en el límite de ruido blanco.

(ii) Condiciones iniciales distribuidas. Caso desacoplado

$$
<t_{i}>_{d}=<t_{i}(\tau=0)>_{d}+\frac{a_{0} \tau}{a_{0}+a}+\mathcal{O}\left(\tau^{2}, D\right),
$$

donde ahora

$$
<t_{i}(\tau=0)>_{d}=\frac{1}{2 a}\left\{\ln \left[\frac{a_{0} a R^{2}}{2\left(a_{0}+a\right) D}\right]+\psi\left(\frac{n}{2}\right)\right\}
$$

(iii) Condiciones iniciales distribuidas. Caso acoplado

$$
<t_{i}>_{a}=\frac{1}{2 a}\left\{\ln \left[\frac{a_{0} a R^{2}}{2\left(a_{0}+a\right) D}\right]+\psi\left(\frac{n}{2}\right)\right\}+\mathcal{O}\left(\tau^{2}, D\right),
$$

el cual coincide con el resulatdo de ruido blanco, dado en la Ec. (2.36). 


\subsection{Los tiempos de relajación no lineales y la teoría cuasi-determinista}

Existe otra escala de tiempo que puede caracterizar de forma completa la relajación dinámica de los momentos estadísticos de los sistemas inestables no lineales definidos en la Ec. (2.6). Esta escala de tiempo mide el tiempo durante el cual los momentos del sistema relajan desde su estado inestable inicial, hasta el estado estacionario estable que le corresponda. Esta escala de tiempo se conoce en la literatura como Tiempo de Relajación No Lineal (TRNL).

La descripción de la dinámica estocástica transitoria a través de TRNL ha sido formulada en términos del formalismo de Fokker-Planck en las referencias $[19,4,5]$, siguiendo una metodología un tanto compleja, en términos de densidades de probabilidad. La conexión de TRNL con la formulación estándar de TCD para caracterizar la dinámica transitoria de sistemas inestables, ya ha sido reportada en la Ref. [23]. Esta descripción es más simple que el formalismo de Fokker-Planck, y permite caracterizar de forma completa cualquier sistema inestable no lineal y éste será el enfoque que adoptaremos en este trabajo.

La escala de tiempo dada por TRNL mide entonces el tiempo durante el cual los momentos estadísticos $\left\langle r^{l}(t)>\right.$ evolucionan desde el valor inicial caracterizado por el valor $<r^{l}(0)>$, hasta su correspondiente valor en el estado estacionario estable caracterizado por el valor $\left\langle r^{l}\right\rangle_{s t}$, donde $r$ es la norma al cuadrado del vector $\mathbf{x}$ definido anteriormente y $l$ es el orden del momento, tal que $l=1,2, \ldots$ Si definimos la cantidad adimensional

$$
m(t)=\frac{\left.<r^{l}(t)>-<r^{l}\right\rangle_{s t}}{\left.<r^{l}(0)>-<r^{l}\right\rangle_{s t}}
$$

Esta función como puede observarse en Fig. 2.1, toma los valores

$$
m(t)=\left\{\begin{array}{ll}
1 & t=0 \\
0 & t=\infty
\end{array} .\right.
$$

Por tanto el área bajo la curva de la Fig. 2.1 se define como el TRNL, dado por

$$
\left.T=\int_{0}^{\infty} m(t) d t=\frac{1}{M_{0}} \int_{0}^{\infty}\left[<r^{l}(t)>-<r^{l}\right\rangle_{s t}\right] d t,
$$

donde $M_{0} \equiv\left\langle r_{0}^{l}\right\rangle-\left\langle r^{l}\right\rangle_{s t}$ con $r_{0} \equiv r(0)$. El calificativo de "no lineal" indica cluc la relajación entre dos estados estacionarios sólo puede darse en sistemas no lineales. Sin 


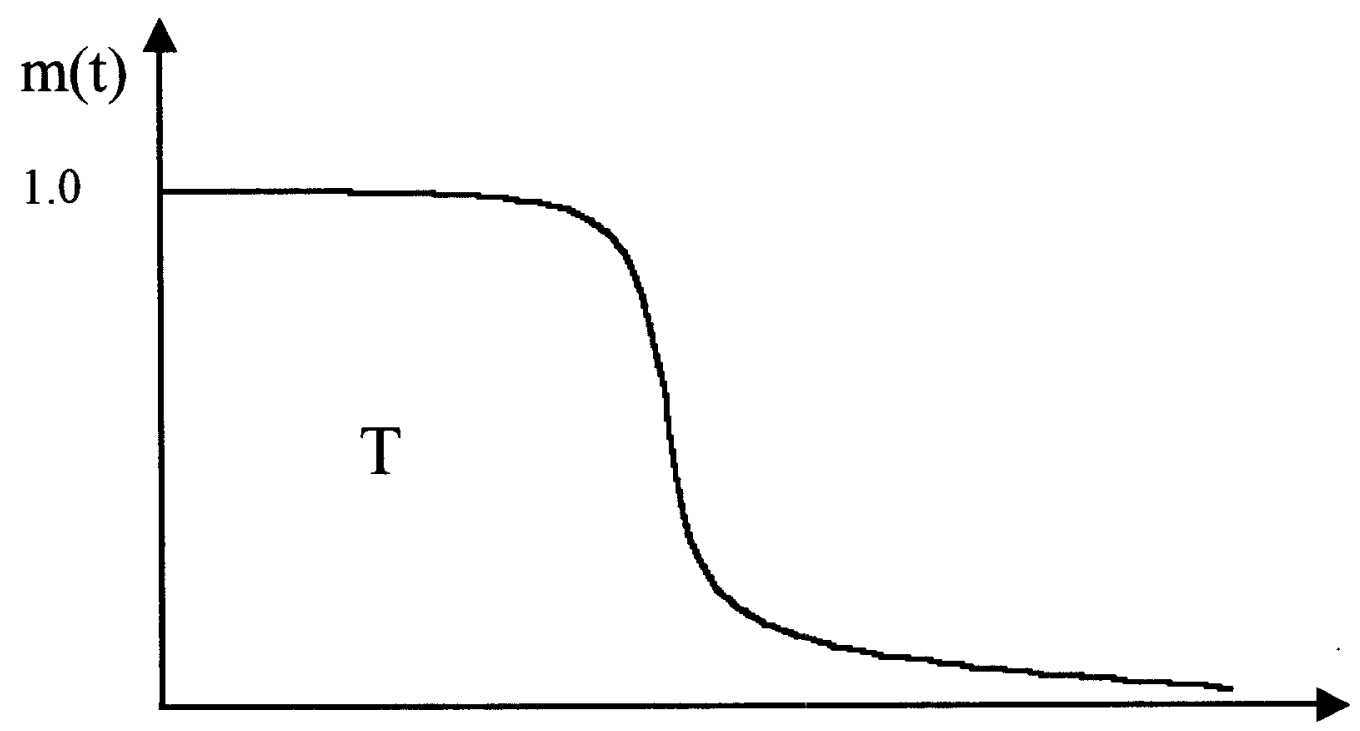

Fig. 2.1: Evolución dinámica de la función $m(t)$

embargo, es posible calcular la escala de tiempo en el régimen lineal de dichos sistemas no lineales a partir de la definición (2.51).

\subsubsection{Caracterización dinámica de sistemas inestables en el ré- gimen lineal}

Antes de caracterizar la relajación de sistemas inestables no lineales mediante TRNL, analicemos primeramente la caracterización dinámica de dichos sistemas en el régimen lineal a través de TRNL y su conexión con TCD. Para lograr esta conexión observemos que el proceso $r(t)$ dado en (2.12), crece indefinidamente una vez que abandona el estado inestable inicial. El crecimiento de este proceso puede detenerse para un cierto valor de referencia $R^{2}$ que podemos hacer coincidir con el valor del estado estacionario $R^{2}=r_{s t}$, como se muestra en la Fig. 2.2. Por lo tanto, el proceso (2.12) incluyendo el orden del momento $l$ podrá escribirse como

$$
r^{l}(t)=h^{2 l} \mathrm{e}^{2 l a t} \theta\left(t_{i}-t\right)+r_{s t}^{l} \theta\left(t-t_{i}\right)
$$

donde $\theta(x)$ es la función escalón de Heaviside. En la Fig. 2.2 se observa el comportamiento promedio $<r^{l}(t)>$.

Si sustituimos (2.52) en (2.51) obtenemos la expresión formal de TRNL asociado al 


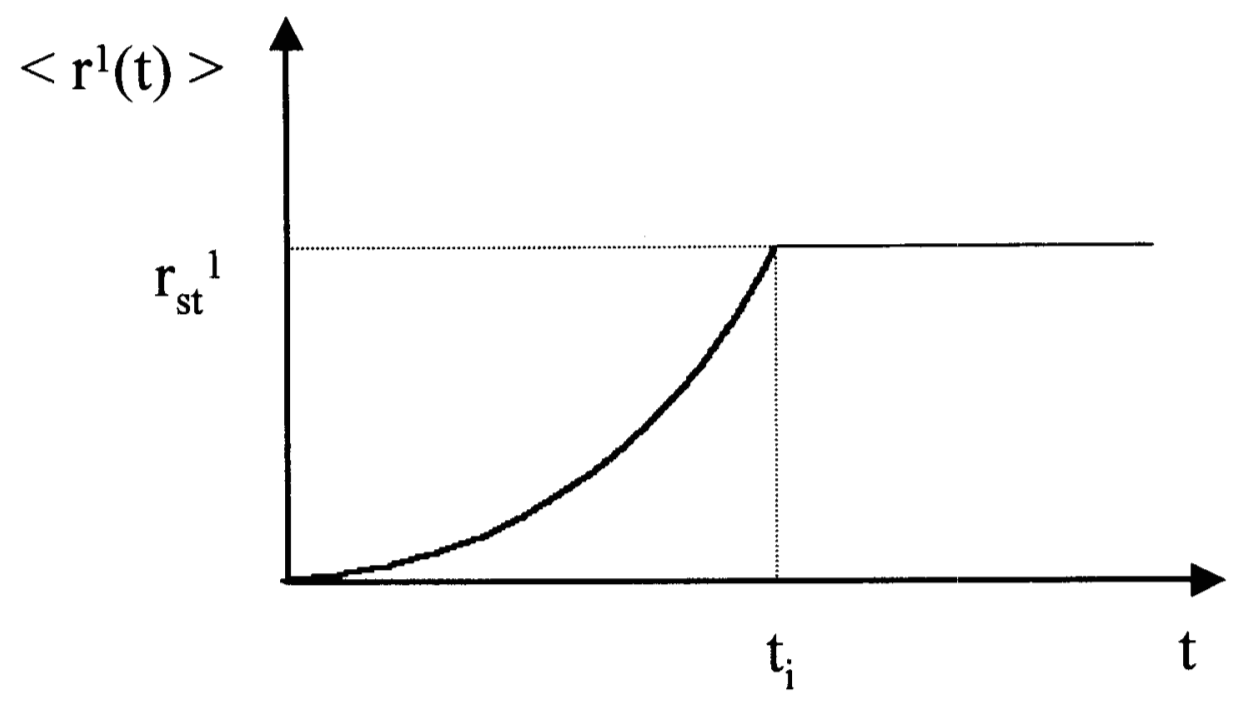

Fig. 2.2: Evolución dinámica de $\left\langle r^{l}(t)\right\rangle$ asociado al proceso (2.52)

momento $\left\langle r^{l}(t)>\right.$ en el régimen lineal

$$
T_{L}=-\frac{r_{s t}^{l}}{2 a M_{0}}\left\langle\ln \left(\frac{r_{s t}}{h^{2}}\right)\right\rangle+C
$$

donde $C=\frac{r_{s t}^{l}}{2 l a M_{0}}\left(1-\frac{\left.<h^{2 l}\right\rangle}{r_{s t}^{l}}\right)$. La Ec. (2.53) es igual a (2.13) excepto la constante $C$, y por tanto su estadística se obtiene de la misma manera como se hizo para obtener el tiempo de paso. Así, en el límite de ruido pequeño podemos mostrar que éste se reduce a

$$
T_{L}=<t_{i}>-\frac{1}{2 a l} .
$$

El primer término de la igualdad corresponde al tiempo de paso (2.32) y el factor $\frac{1}{2 a l}$ es la contribución de los TRNL en el régimen lineal, el cual contiene la contribución del orden del momento.

\subsubsection{Caracterización dinámica de sistemas inestables en el ré- gimen no lineal}

Hagamos ahora la conexión de TRNL con TCD tomando en cuenta las contribuciones no lineales del sistema (2.6). Para estos sistemas no necesitamos imponer valores de referencia preestablecidos en su evolución dinámica, puesto que las no linealidades son una barrera natural. Para realizar la conexión correspondiente con TCD, debemos recordar que TC'D 
nos dice que la condición inicial es una variable aleatoria $r(0)=h^{2}$. Sabemos también que para tiempos largos el valor $r(t \rightarrow \infty)=r_{s t}$. El siguiente paso consiste en transformar la definición de TRNL en una cuadratura en términos de la variable $r$. Esto puede lograrse si tomamos en cuenta la Ec. (2. 1), que nos dice que $\dot{r}=f(r)$ y por lo tanto

$$
T_{N L}=\frac{1}{M_{0}}\left\langle\int_{r_{0}=h^{2}}^{r_{s t}} \frac{\left(r^{l}-r_{s t}^{l}\right)}{f(r)} d r\right\rangle .
$$

Lo siguiente es identificar a $\dot{r}=f(r)$ con la parte determinista de $(2.6)$ en términos de la variable $r$, es decir

$$
\dot{r}=2 a r+2 r N(r),
$$

la cual debe ser compatible con (2.1) dependiendo de la forma explícita de la función $N(r)$. De manera que sin perder generalidad podemos usar la expresión (2.1) y sustituirla en la Ec. (2.55) para obtener

$$
T_{N L}=-\frac{r_{s t}^{l}}{2 a M_{0}}\left\langle\ln \left(\frac{r_{s t}}{h^{2}}\right)\right\rangle+I_{N L}
$$

donde

$$
I_{N L} \equiv-\frac{r_{s t}^{l-1}}{M_{0}}\left\langle\int_{r_{0}=h^{2}}^{r_{s t}}\left[g(r)(1+S(r))+C_{0} \frac{S(r)}{r}\right] d r\right\rangle,
$$

tal que $S(r)$ es un polinomio de la forma $S(r)=\sum_{k=1}^{l-1}\left(\frac{r}{r_{s t}}\right)^{k}$. La expresión (2.58) corresponde a las contribuciones no lineales del sistema, y su forma explícita depende del término $N(r)$.

Finalmente el tiempo de relajación no lineal (2.57) asociado a los momentos $\left\langle r^{l}(t)\right\rangle$ de la dinámica (2.6) en el límite de ruido pequeño, puede escribirse también como

$$
T_{N L}=<t_{i}>+I_{N L} \text {. }
$$

El primer término corresponde al tiempo de paso (2.32) del sistema lineal y el segundo término $I_{N L}$ da cuenta de las contribuciones no lineales del sistema.

\subsubsection{Los TRNL inducidos por ruido blanco gaussiano}

Para este caso podemos concluir que la caracterización dinámica del sitema (2.6) en el régimen lineal a través de TRNL está dada por (2.54), es decir

$$
T_{L}=<t_{i}>-\frac{1}{2 a l}
$$




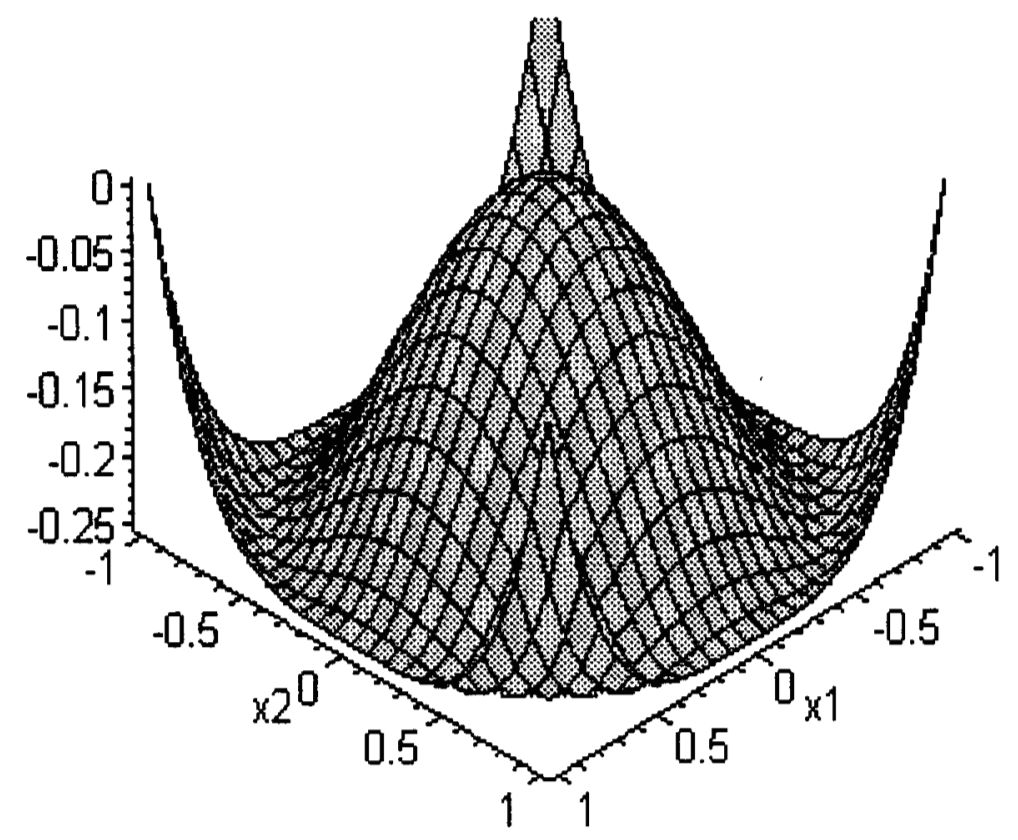

Fig. 2.3: Visualización del potencial $V\left(x_{1}, x_{2}\right)$ en el espacio de tres dimensiones

donde $\left\langle t_{i}>\right.$ es el tiempo de paso dado en (2.36).

El correspondiente TRNL en el régimen no lineal, de acuerdo con (2.59) es igual a

$$
T_{N L}=<t_{i}>+I_{N L}
$$

siendo $<t_{i}>$ nuevamente el tiempo de paso, dado en (2.36).

\subsubsection{Los TRNL inducidos por ruido de color gaussiano}

En el régimen lineal los $T_{L}$ tienen las mismas expresiones que las obtenidas en los tres casos anteriores para los tiempos de paso, excepto que ahora las expresiones (2.45), (2.47) y (2.48) se les deben restar el factor $\frac{1}{2 a l}$.

Para los sistemas no lineales los $T_{N L}$ en los tres casos, de acuerdo con (2.59), estarán dados por (2.44): (2.46) y (2.48) más la contribución no lineal $I_{N L}$.

\subsubsection{Modelo de Landau}

Apliquemos ahora el formalismo anterior para calcular el TRNL del modelo de Landau, el cual corresponde al modelo de potencial biestable para el caso de una variable [23]. El potencial de este modelo para el caso de dos variables independientes $V\left(x_{1}, x_{2}\right)$, se muestra en la Fig. 2.3. Calculemos $T_{N L}$ para el modelo mostrado en la Fig. 2.3. En 
este caso las ecuaciones tipo Langevin con ruido aditivo que describen la dinámica de este sistema puede escribirse como

$$
\begin{aligned}
& \dot{x}_{1}=a x_{1}-c r x_{1}+\xi_{1}(t), \\
& \dot{x}_{2}=a x_{2}-c r x_{2}+\xi_{2}(t) .
\end{aligned}
$$

De acuerdo con (2.6) se verifica que la contribución no lineal $N(r)=-c r$, siendo $r=x_{1}^{2}+x_{2}^{2}$. El término fluctuante $\xi_{i}(t)$ puede satisfacer las propiedades de ruido blanco o ruido de color gaussianos.

Para obtener el $T_{N L}$ de (2.61) debemos construir la ecuación determinista asociada a la variable $r$, es decir

$$
\dot{r}=2 a r-2 c r^{2}=\frac{2 a r\left(r_{s t}-r\right)}{r_{s t}} .
$$

De donde podemos concluir que $r_{s t}=\frac{a}{c}, g(r)=0$ y $C_{0}=\frac{r_{s t}}{2 a}$. Por lo tanto los TRNL en el régimen no lineal asociados a los momentos $\left\langle r^{l}(t)\right\rangle$ será

$$
T_{N L}=\frac{1}{2 a}\left\{\ln \left(\frac{r_{s t}}{2 \sigma^{2}}\right)-\psi(1)+\psi(l)+\gamma\right\},
$$

donde $\sigma^{2}$ será igual que (2.26) y su forma explícita dependerá del tipo de ruido que se esté analizando y $\gamma=0.577$ es la constante de Euler. Para este modelo particular la contribución de los TRNL en el régimen no lineal está dada por $I_{N L}=\frac{1}{2 a}[(\psi(l)+\gamma]$.

\subsection{Formulación Estándar de TCD en presencia de fuerza externa constante}

Nuestro propósito en este apartado es estudiar la dinámica (2.6) en presencia de fuerza externa constante, tal que

$$
\dot{\mathbf{x}}=a \mathbf{x}+N(r) \mathbf{x}+\mathbf{F}_{e}+\mathbf{z}(t)
$$

el tercer término es la fuerza externa constante $\mathbf{F}_{e}$, con elementos $f_{e_{i}}$. En esta sección sólo vamos a trabajar con ruido blanco gaussiano, cuyas propiedades ya han sido definidas en la Sec. 2.3.3. 
El análisis de TCD inicia con la parte lineal de la Ec. (2.65) o sea

$$
\dot{\mathbf{x}}=a \mathbf{x}+\mathbf{F}_{e}+\mathbf{z}(t)
$$

siendo la solución formal

$$
\mathbf{x}(t)=\mathrm{e}^{a t} \mathbf{h}(t)
$$

donde

$$
\mathbf{h}(t)=\int_{0}^{t} \mathrm{e}^{-a s}\left[\mathbf{F}_{e}+\mathbf{z}(s)\right] d s,
$$

dado que asumimos condiciones iniciales fijas, es decir $\mathbf{x}(0)=0$.

De la misma forma como se hizo en la Sec. 2.3.1, podemos garantizar que en el límite de tiempos largos, tales que at $\gg 1$, el proceso (2.68) se convierte en una VAG, puesto que para valores pequeños de los elementos de $\mathbf{F}_{e}$ y de $\mathbf{z}(t)$ se garantiza que

$$
\lim _{t \rightarrow \infty} \frac{d \mathbf{h}(t)}{d t}=\lim _{t \rightarrow \infty} \mathrm{e}^{-a t}\left[\mathbf{F}_{e}+z(t)\right] \rightarrow 0 .
$$

Por lo tanto $\mathbf{h}(\infty)$ se convierte en una VAG que definimos por $\mathbf{h}$, con elementos $h_{i}$ constantes que son también variables aleatorias gaussianas. Bajo estas condiciones el proceso (2.67) se convierte en un proceso cuasi-determinista, que en términos de la variable $r$ se puede escribir como

$$
r(t)=h^{2} \mathrm{e}^{2 a t}
$$

donde la variable $h^{2} \equiv \mathbf{h}^{T} \mathbf{h}=h_{1}^{2}+\cdots+h_{n}^{2}$.

De esta última ecuación se obtiene la expresión de los tiempos de paso que es igual a (2.13), es decir

$$
<t_{i}>=\frac{1}{2 a}\left\langle\ln \left(\frac{R^{2}}{h^{2}}\right)\right\rangle .
$$

Para calcular $\left\langle t_{i}>\right.$ requerimos de la estadística de las variables $\left(h_{1}, \cdots, h_{n}\right)$, la cual se obtiene de la densidad de probabilidad conjunta $P\left(h_{1}, \ldots, h_{n}\right)$. Para poder calcular esta densidad de probabilidad requerimos que la matriz $\sigma_{i j} \equiv<h_{i} h_{j}>-<h_{i}><h_{j}>$ sea diagonal.

Para este caso, el valor promedio de $\left\langle h_{i}(t)\right\rangle$ es diferente de cero

$$
<h_{i}(t)>=\int_{0}^{t} \mathrm{e}^{-a s} f_{e i} d s=\frac{f_{e i}}{a}\left(1-\mathrm{e}^{-a t}\right) .
$$


Con ayuda de la Ec. (2.68) tenemos que la función de correlación se puede escribir, como

$$
<h_{i}(t) h_{j}(t)>=K_{e}(t)+K_{R}(t)
$$

donde

$$
\begin{array}{r}
K_{e}(t) \equiv \int_{0}^{t} \int_{0}^{t} \mathrm{e}^{-a\left(s+s^{\prime}\right)}\left(s^{\prime}\right) f_{e i} f_{e j} d s d s^{\prime}=<h_{i}(t)><h_{j}(t)> \\
K_{R}(t) \equiv \int_{0}^{t} \int_{0}^{t} \mathrm{e}^{-a\left(s+s^{\prime}\right)}<\xi_{i}(s) \xi_{j}\left(s^{\prime}\right)>d s d s^{\prime}=\frac{D}{a}\left(1-\mathrm{e}^{-2 a t}\right) \delta_{i j} .
\end{array}
$$

Con todos estos resultados podemos mostrar que en el límite de tiempos largos, la matriz $\sigma_{i j}=\frac{D}{a} \delta_{i j}$ es diagonal, tal que $\sigma_{i i} \equiv \sigma^{2} \mathrm{y}$

$$
\sigma^{2}=\frac{D}{a}
$$

Este resultado es igual a (2.35), pero sin el término $\frac{D}{a_{0}}$ ya que nuestro análisis es con condiciones iniciales fijas.

Bajo estas condiciones tenemos que la densidad de probabilidad conjunta viene dada por

$$
P\left(h_{1}, \ldots, h_{n}\right)=\frac{1}{\left(2 \pi \sigma^{2}\right)^{n / 2}} \mathrm{e}^{-\alpha^{2}\left[\left(h_{1}-<h_{1}>\right)^{2}+\cdots+\left(h_{n}-<h_{n}>\right)^{2}\right]} .
$$

La densidad de probabilidad marginal $P(h)$ se construye con la ayuda de un nuevo espacio de variables definido como $\mathbf{u}=\left(u_{1}, \ldots, u_{n}\right)$ y usando la transformación

$$
P\left(h_{1}, \ldots, h_{n}\right) d h_{1} \ldots d h_{n} \rightarrow P\left(u_{1}, \ldots, u_{n}\right) d V
$$

siendo $d V=J(\mathbf{u}) d \mathbf{u}$ el elemento de volumen en el espacio u y $J(\mathbf{u})$ es el jacobiano de la transformación. Si hacemos $u_{1}=h$, entonces la densidad de probabilidad conjunta en este espacio, será

$$
P\left(h, u_{2}, \ldots, u_{n}\right) d V=\text { cte } \exp \left[-\alpha^{2}\left(h^{2}+q^{2}-2 \mathbf{q} \cdot \mathbf{h}\right)\right] d V,
$$

donde hemos definido el vector $\mathbf{q} \equiv\left(<h_{1}>, \ldots,<h_{n}>\right)$. Finalmente la $P(h)$ puede ser obtenida de (2.79) calculando el jacobiano y integrando sobre el resto de las variables $\left(u_{2}, \ldots, u_{n}\right)$. 


\subsubsection{Sistemas de dos variables}

Para los sistemas de dos variables la densidad de probabilidad marginal está dada por

$$
P(h)=2 \alpha^{2} h I_{0}\left(2 \alpha^{2} h q\right) \mathrm{e}^{-\alpha^{2}\left(h^{2}+q^{2}\right)},
$$

donde

$$
q^{2}=\frac{\left|\mathbf{F}_{e}\right|^{2}}{a^{2}}
$$

siendo $\left|\mathbf{F}_{e}\right|^{2}$ la amplitud al cuadrado de la fuerza externa, tal que $\left|\mathbf{F}_{e}\right|^{2}=f_{e_{1}}^{2}+f_{e_{2}}^{2}$. La función $I_{0}(x)$ es la función modificada de Bessel de orden cero.

Con ayuda de (2.13) y (2.80) obtenemos la expresión de los tiempos de paso

$$
<t_{i}>=<t_{i}>_{0}+\frac{1}{2 a} \psi(1)-\frac{\mathrm{e}^{\beta^{2}}}{2 a} \sum_{m=0}^{\infty} \frac{\beta^{2 m}}{m !} \psi(m+1)+\mathcal{O}(D)+\mathcal{O}\left(q^{2}\right)
$$

donde definimos el parámetro $\beta^{2} \equiv(\alpha q)^{2} \mathrm{y}<t_{i}>_{0}$ es el tiempo de paso en ausencia de fuerza externa, dado por

$$
<t_{i}>_{0}=\frac{1}{2 a}\left\{\ln \left(\alpha^{2} R^{2}\right)-\psi(1)\right\} .
$$

En el Apéndice C.1 demostramos que la serie de (2.82) puede simplificarse en una expresión más simple, con lo que TRNL en el régimen lineal, se puede escribir como

$$
T_{L}=T_{L}^{0}+\frac{1}{2 a} \sum_{m=1}^{\infty} \frac{(-1)^{m} \beta^{2 m}}{m m !}+\mathcal{O}(D)+\mathcal{O}\left(q^{2}\right)
$$

donde $T_{L}^{0}$ es TRNL en ausencia de fuerza externa, tal que

$$
T_{L}^{0}=\frac{1}{2 a}\left\{\ln \left(\alpha^{2} r_{s t}\right)-\psi(1)-\frac{1}{l}\right\}
$$

Entonces TRNL en el régimen no lineal de (2.65) es igual a

$$
T_{N L}=T_{N L}^{0}+\frac{1}{2 a} \sum_{m=1}^{\infty} \frac{(-1)^{m} \beta^{2 m}}{m m !},
$$

siendo $T_{N L}^{0}$ el tiempo de relajación no lineal en ausencia de fuerza externa, dada por

$$
T_{N L}^{0}=<t_{i}>_{0}+\mathbf{I}_{N L}
$$

donde $\mathbf{I}_{N L}$ contiene las contribuciones no lineales de (2.65), que se obtiene con ayuda de (2.58). 


\subsubsection{Sistemas de tres variables}

En este caso, la densidad de probabilidad marginal $P(h)$ se obtiene con ayuda de (2.79), tal que

$$
P(h)=\frac{2 \alpha}{\sqrt{\pi} q} h \sinh \left(2 \alpha^{2} q\right) e^{-\alpha^{2}\left(h^{2}+q^{2}\right)},
$$

nuevamente

$$
q^{2}=\frac{\left|\mathbf{F}_{e}\right|^{2}}{a^{2}}
$$

esta ecuación tiene la misma estructura matemática que la Ec. (2.81) pero no es igual, dado que ahora $\left|\mathbf{F}_{e}\right|^{2}=f_{e_{1}}^{2}+f_{e_{2}}^{2}+f_{e_{3}}^{2}$.

Para este caso tenemos que los timpos de paso, están dados por

$$
<t_{i}>=<t_{i}>_{0}+\frac{1}{2 a} \psi\left(\frac{3}{2}\right)-\frac{\mathrm{e}^{-\beta^{2}}}{2 a} \sum_{m=0}^{\infty} \frac{\beta^{2 m}}{m !} \psi\left(m+\frac{3}{2}\right)+\mathcal{O}(D)+\mathcal{O}\left(q^{2}\right)
$$

donde

$$
<t_{i}>_{0}=\frac{1}{2 a}\left\{\ln \left(\alpha^{2} R^{2}\right)-\psi\left(\frac{3}{2}\right)\right\} .
$$

También la serie de (2.90) se puede simplificar a una expresión más sencilla, como se demuestra en el Apéndice C.2, entonces los TRNL en el régimen lineal se pueden expresar, como

$$
T_{L}=T_{L}^{0}+\frac{\sqrt{\pi}}{2 a} \sum_{m=1}^{\infty} \frac{(-1)^{m} \beta^{2 m}}{2 m\left(m+\frac{1}{2}\right) !}+\mathcal{O}(D)+\mathcal{O}\left(q^{2}\right)+\mathcal{O}\left(D q^{2}\right)
$$

donde

$$
T_{L}^{0}=\frac{1}{2 a}\left\{\ln \left(\alpha^{2} r_{s t}\right)-\psi\left(\frac{3}{2}\right)-\frac{1}{l}\right\} .
$$

Por último los TRNL en el régimen no lineal, son iguales a

$$
T_{N L}=T_{N L}^{0}+\frac{\sqrt{\pi}}{2 a} \sum_{m=1}^{\infty} \frac{(-1)^{m} \beta^{2 m}}{2 m\left(m+\frac{1}{2}\right) !}
$$

con

$$
T_{N L}^{0}=<t_{i}>_{0}+\mathbf{I}_{N L}
$$

donde $\mathbf{I}_{N L}$ contiene las contribuciones no lineales del sistema. 


\section{Capítulo 3}

\section{Caracterización dinámica de sistemas inestables rotacionales. Formulación Matricial}

La contribución original de nuestro trabajo inicia en este capítulo, ya que nuestro propósito es caracterizar el proceso de decaimiento dinámico de los sistemas inestables rotacionales, propuesto en la Ec. (1.22) en ausencia de fuerza externa.

Para este tipo de sistemas estamos también interesados en caracterizar el proceso de decaimiento a través de los tiempos de paso y de los tiempos de relajación no lineales, siguiendo la metodología de Teoría Cuasi-Determinista (TCD) de la formulación estándar en términos ahora de una formulación matricial generalizada. En este sentido nuestra descripción del problema, se llevará a cabo guiandonos con las mismas ideas físicas involucradas en la descripción de TCD.

La ecuación tipo Langevin asociada al vector columna $\mathbf{x}$ que describe la dinámica de sistemas inestables rotacionales en ausencia de fuerza externa, puede escribirse de la siguiente forma

$$
\dot{\mathbf{x}}=a \mathbf{x}+\mathbf{W x}+N(r) \mathbf{x}+\mathbf{z}(t),
$$

donde el término $\mathbf{W x}$ es la nueva contribución con respecto al esquema de (2.6), siendo $\mathrm{W}$ una matriz real antisimétrica tal que $\mathrm{W}^{T}=-\mathrm{W}$ y $\mathbf{W}^{T}$ su matriz transpuesta. A diferencia de la formulación estándar el conjunto de variables $\mathbf{x}$ ya no son independientes sino que están acopladas debido a la matriz $\mathbf{W}$, la cual da cuenta de los efectos de rotación del sistema. Como puede observarse la fuerza sistemática lineal $\mathbf{F}$ es la suma de dos contribuciones, una es la parte conservativa $\mathbf{F}_{c}=a \times$ ya que $\nabla \times \mathbf{F}_{c}=0$ y la otra 
es la parte no conservativa $\mathbf{F}_{n c}=\mathbf{W x}$ puesto que $\nabla \times \mathbf{F}_{n c} \neq 0$ y por lo tanto $\mathbf{F}$ no es derivable de un potencial.

En el Apéndice A.2, se muestra que toda matriz antisimétrica en el espacio de tres dimensiones determina un vector $\vec{w}$ tal que el producto matricial de la matriz antisimẹtrica es igual al producto vectorial del vector $\vec{w}$, que representa al vector velocidad angular. De manera que para los sistemas de dos y tres variables que estudiaremos en esta tesis, el producto $\mathbf{W x}$ siempre es posible escribirlo como el producto vectorial de dos vectores a saber $\mathbf{W} \mathbf{x}=\vec{w} \times \mathbf{x}$. Esta relación nos indica entonces una rotación del sistema en un plano perpendicular al vector $\vec{w}$.

La parte lineal de la Ec. (3.1) es matemáticamente muy similar a las ecuaciones de Langevin generalizadas propuestas por Fox y Uhlebeck en su trabajo citado en la Ref. [12]. Dicha ecuación generalizada tiene sus fundamentos en las ecuaciones de movimiento microscópicas que son invariantes ante inversión y traslación temporal, como se muestra en el Apéndice B. La parte lineal asociadad a la Ec. (3.1) describe una situación física distinta a la propuesta por Fox y Uhlebeck. Sin embargo, la Ec. (3.1) puede en principio obtenerse a partir de la propuesta de Fox, mediante un cambio repentino en los parámetros involucrados en la matriz simétrica de la ecuación de langevin generalizada.

\subsection{Formulación Matricial de la teoría cuasi-deter- minista}

\subsubsection{Ruido blanco gaussiano}

Supongamos que la fuerza flutuante de la Ec. (3.1) satisface las propiedades de ruido blanco, con valor medio $\left\langle\xi_{i}(t)\right\rangle=0$ y función de correlación

$$
<\xi_{i}(t) \xi_{j}\left(t^{\prime}\right)>=2 Q_{i j} \delta_{i j} \delta\left(t-t^{\prime}\right), \quad i, j=1, \ldots, n,
$$

donde $Q_{i j}$ es una matriz que representa a la intensidad del ruido.

De nueva cuenta, el análisis de TCD inicia con la aproximación lineal de (3.1), esto es

$$
\dot{\mathbf{x}}=a \mathbf{x}+\mathbf{W} \mathbf{x}+\mathbf{z}(t),
$$

cuya solución es

$$
\mathbf{x}(t)=\mathrm{e}^{a t} \mathrm{e}^{\mathbf{W} t} \mathbf{h}(t)
$$


donde $\mathbf{h}(t)$ está definida por

$$
\mathbf{h}(t)=\mathbf{x}(0)+\mathbf{g}(t), \quad \mathbf{g}(t)=\int_{0}^{t} \mathrm{e}^{-a s} \mathrm{e}^{-\mathbf{W} s} \mathbf{z}(s) d s .
$$

Como se muestra en el Apéndice A.1, el factor $\mathrm{e}^{-\mathbf{W} t}=\mathbf{R}(t)$ es una matriz ortogonal de rotación ya que su transpuesta es igual a su matriz inversa, es decir $\mathbf{R}^{T}(t)=\mathbf{R}^{-1}(t)$.

Siguiendo las ideas de TCD, decimos que para valores pequeños de los elementos del vector $\mathbf{z}(t)$ garantizamos que en límite de tiempos largos

$$
\lim _{t \rightarrow \infty} \frac{d \mathbf{h}(t)}{d t}=\lim _{t \rightarrow \infty} \mathrm{e}^{-a t} \mathbf{R}(t) \mathbf{z}(t) \rightarrow 0,
$$

y por tanto el proceso $\mathbf{h}(\infty)=\mathbf{h}$ es un vector cuyos elementos $h_{i}=x_{0 i}+g_{i}$ son VAG. Así el proceso (3.4) se transforma en un proceso cuasi-determinista, tal que la norma al cuadrado satisface lo siguiente

$$
r(t)=\mathbf{x}^{T} \mathbf{x}=h^{2} e^{2 a t}
$$

donde $h^{2} \equiv \mathbf{h}^{T} \mathbf{h}=h_{1}^{2}+\cdots+h_{n}^{2}$. Esto nos muestra que $r(t)$ satisface el mismo proceso que el de la formulación estándar. Así que el tiempo de paso para alcanzar el valor de referencia $R^{2}$ es también

$$
<t_{i}>=\frac{1}{2 a}\left\langle\ln \left(\frac{R^{2}}{h^{2}}\right)\right\rangle,
$$

por lo que sus propiedades estadísticas se obtienen siguiendo la misma metodología del capítulo anterior.

En este esquema matricial, la densidad de probabilidad conjunta para las variables gaussianas satisface la expresión general

$$
P\left(h_{1}, \ldots, h_{n}\right)=\text { cte } \exp \left[-\frac{1}{2} \sum_{i, j}\left(\sigma^{-1}\right)_{i j}\left(h_{i}-<h_{i}>\right)\left(h_{j}-<h_{j}>\right)\right]
$$

siendo la constante cte $\equiv \frac{1}{(2 \pi)^{n / 2}\left(\text { Det } \sigma_{i j}\right)^{1 / 2}}$. Si la matriz $\sigma_{i j}$ es simétrica $\sigma_{i j}=\sigma_{j i}$ y definida positiva, entonces la matriz inversa $\left(\sigma^{-1}\right)_{i j}=\left(\sigma^{-1}\right)_{j i}$ y su raíz cuadrada $\left(\sigma^{1 / 2}\right)_{i j}=\left(\sigma^{1 / 2}\right)_{j i}$, así como su raíz cuadrada inversa $\left(\sigma^{-1 / 2}\right)_{i j}=\left(\sigma^{-1 / 2}\right)_{j i}$ existen [49].

Como podemos ver, para construir $P\left(h_{1}, \ldots, h_{n}\right)$ requerimos del cálculo de $\sigma_{i j}$, que esta definida por

$$
\sigma_{i j} \equiv<h_{i} h_{j}>-<h_{i}><h_{j}>\equiv \sigma_{i j}^{0}+\sigma_{i j}^{R}
$$


donde

$$
\sigma_{i j}^{0} \equiv<x_{i}(0) x_{j}(0)>-<x_{i}(0)><x_{j}(0)>, \quad \sigma_{i j}^{R} \equiv<g_{i} g_{j}>-<g_{i}><g_{j}>
$$

La matriz $\sigma_{i j}^{0}$ da cuenta de las fluctuaciones de las condiciones iniciales y la matriz $\sigma_{i j}^{R}$ da cuenta de las fluctuaciones internas causadas por el ruido.

Para calcular la matriz $\sigma_{i j}$ se requiere del conocimiento de $\left\langle h_{i}(t)\right\rangle=\left\langle x_{i}(0)>\right.$ y de la función de correlación $\left\langle h_{i}(t) h_{j}(t)\right\rangle$, tal que

$$
<h_{i}(t) h_{j}(t)>=K_{i j}^{0}+K_{i j}^{R}(t)
$$

donde

$$
K_{i j}^{0} \equiv<x_{i}(0) x_{j}(0)>, \quad K_{i j}^{R}(t) \equiv<g_{i}(t) g_{j}(t)>
$$

y

$$
K_{i j}^{R}(t)=\sum_{k, m} \int_{0}^{t} \int_{0}^{t} \mathrm{e}^{-a\left(s+s^{\prime}\right)} R_{i k}(s) R_{j m}\left(s^{\prime}\right)<\xi_{k}(s) \xi_{m}\left(s^{\prime}\right)>d s d s^{\prime} .
$$

Para calcular el primer término de (3.12) recurrimos otra vez a la preparación de las condiciones iniciales en $t=0$. Esta situación debe establecerse mediante una dinámica para tiempos $t<0$ siguiendo las ideas de la formulación estándar. En este sentido, la dinámica del estado estacionario se describe por

$$
\dot{\mathbf{x}}_{0}=-a_{0} \mathbf{x}_{0}+\mathbf{W} \mathbf{x}_{0}+\mathbf{z}(t)
$$

siendo $a_{0}>0$ y $\mathbf{z}(t)$ cumple con las mismas propiedades de ruido blanco cuya intensidad puede ser $Q_{i j}^{\prime}$. Consideremos por sencillez que $Q_{i j}^{\prime}=Q_{i j}$. La solución de (3.15) es

$$
\mathbf{x}_{0}=\int_{-\infty}^{0} \mathrm{e}^{a_{0} s} \mathbf{R}\left(s^{\prime}\right) \mathbf{z}\left(s^{\prime}\right) d s^{\prime}
$$

La situación física es similar al caso estándar, e.d. en el instante $t=0$ el sistema sufre un cambio brusco en el parámetro de control, de $-a_{0} \rightarrow a$ y deja al sistema inicialmente localizado alrededor de un estado inestable.

Así, el valor promedio de $\left\langle x_{i}(0)\right\rangle=0$ y su función de correlación $K_{i j}^{0}$ viene dado por

$$
K_{i j}^{0} \equiv 2 \int_{-\infty}^{0} \int_{-\infty}^{0} \mathrm{e}^{a_{0}\left(s+s^{\prime}\right)} \sum_{k} R_{i k}(s) R_{j k}\left(s^{\prime}\right) Q_{k k} \delta\left(s-s^{\prime}\right) d s d s^{\prime} .
$$

Si pedimos que los elementos de la matriz $Q_{k k}=Q$ y tomamos en cuenta las propiedardes de la matriz de rotación, entonces $K_{i j}^{0}=\frac{Q}{a_{0}} \delta_{i j}$. Por lo tanto la matriz de las condicionesi 
iniciales $\sigma_{i j}^{0}=\frac{Q}{a_{0}} \delta_{i j}$ es diagonal, es decir $\sigma_{i i}^{0}=\sigma_{0}^{2}=\frac{Q}{a_{0}}$. En este caso la densidad de probabilidad conjunta, será

$$
P_{s t}\left(x_{0_{1}}, \ldots, x_{0 n}\right)=\operatorname{cte} \mathrm{e}^{-\alpha^{2}\left(x_{0_{1}}^{2}+\cdots+x_{0 n}^{2}\right)},
$$

con $\alpha_{0}^{2} \equiv \frac{1}{2 \sigma_{0}^{2}}$.

Por otro lado $K_{i j}^{R}(t)$ puede reducirse a

$$
K_{i j}^{R}(t)=2 \int_{0}^{t} \int_{0}^{t} \mathrm{e}^{-a\left(s+s^{\prime}\right)} \sum_{k} R_{i k}(s) R_{j k}\left(s^{\prime}\right) Q_{k k} \delta\left(s-s^{\prime}\right) d s d s^{\prime}
$$

y de acuerdo con las propiedades de ortoganlidad de la matriz de rotación, se tiene que

$$
K_{i j}^{R}(t)=\frac{Q}{a}\left(1-\mathrm{e}^{-2 a t}\right) \delta_{i j}
$$

Entonces en el límite de tiempos largos $K_{i j}^{R}(\infty)=\frac{Q}{a} \delta_{i j} \mathrm{y}<g_{i}(t)>=0$. En consecuencia la matriz $\sigma_{i j}^{R}=\frac{Q}{a} \delta_{i j}$ es diagonal, con elementos $\sigma_{i i}^{R} \equiv \sigma_{R}^{2}=\frac{Q}{a}$, y por lo tanto la matriz (3.10) también es diagonal con elementos $\sigma_{i i}=\sigma_{i i}^{0}+\sigma_{i i}^{R}=\sigma^{2}$ siendo ,

$$
\sigma^{2}=\left(\frac{1}{a_{0}}+\frac{1}{a}\right) Q
$$

En estas circunstancias el conjunto de variables $h_{i}$ son independientes y por lo tanto la densidad de probabilidad conjunta para las variables $h_{i}$, es igua a

$$
P\left(h_{1}, \ldots, h_{n}\right)=\text { cte } \mathrm{e}^{-\alpha^{2}\left(h_{1}^{2}+\cdots+h_{n}^{2}\right)},
$$

siendo $\alpha^{2} \equiv \frac{1}{2 \sigma^{2}}$.

Por lo tanto, la densidad de probabilidad marginal, será

$$
P(h)=\frac{2 \alpha^{n}}{\Gamma\left(\frac{n}{2}\right)} h^{n-1} \mathrm{e}^{-\alpha^{2} h^{2}} .
$$

Análogamente para las variables $x_{i}(0)$, es decir

$$
P_{s t}\left(x_{0}\right)=\frac{2 \alpha_{0}^{n}}{\Gamma\left(\frac{n}{2}\right)} x_{0}^{n-1} \mathrm{e}^{-\alpha_{0}^{2} x_{0}^{2}} .
$$

Como consecuencia del formalismo anterior podemos verificar que el tiempo de paso. y el TRNL en el régimen lineal están dadas respectivamente por

$$
<t_{i}>=\frac{1}{2 a}\left\{\ln \left[\frac{a a_{0} R^{2}}{2\left(a_{0}+a\right) Q}\right]-\psi\left(\frac{n}{2}\right)\right\} .
$$




$$
T_{L}=<t_{i}>-\frac{1}{2 a l} .
$$

Para calcular el tiempo de relajación no lineal $T_{N L}$, podemos verificar de (3.1) que la ecuación determinista asociada a la variable $r$ tiene la misma estructura de (2.56), por lo tanto

$$
T_{N L}=<t_{i}>+I_{N L}
$$

De acuerdo con estos resultados, podemos concluir que las escalas de tiempo (3.25), (3.26) y (3.27) que caracterizan a la dinámica rotacional (3.1) son exactamente las mismas escalas de tiempo dadas en las Ecs. (2.36) (2.60) y (2.61) del Cap. 2 respectivamente, siempre y cuando $Q=D$. Antes de dar la explicación del porqué de esta igualdad en las escalas de tiempo, estudiemos ahora la caracterización dinámica de (3.1) en el caso de ruido de color gaussiano.

\subsubsection{Ruido de color gaussiano}

Supongamos ahora que la fuerza fluctuante de la dinámica (3.1) satisface las propiedades de ruido de color gaussiano, con valor medio $\left\langle\xi_{i}(t)\right\rangle=0$ y función de correlación

$$
<\xi_{i}(t) \xi_{j}\left(t^{\prime}\right)>=\frac{Q_{i j}}{\tau} \delta_{i j} \mathrm{e}^{-\frac{\left|t-t^{\prime}\right|}{\tau}}, \quad i, j=1, \ldots, n
$$

donde $Q_{i j}$ es una matriz que representa a la intensidad del ruido.

El tiempo de paso satisface la misma expresión dada en la Ec. (3.8) de la sección

previa, así que de acuerdo con el análisis hecho en el caso de ruido blanco, para calcular la densidad de probabilidad conjunta (3.9) requerimos de las propiedades de la matriz $\sigma_{i j}$ definida por

$$
\sigma_{i j} \equiv<h_{i} h_{j}>-<h_{i}><h_{j}>=\sigma_{i j}^{0}+\sigma_{i j}^{R}+\sigma_{i j}^{S R}
$$

los dos primeros terminos de esta igualdad ya han sido defininidos en la Ec. (3.11), entonces el tercer término se define como

$$
\sigma_{i j}^{S R} \equiv<x_{0 i} g_{j}>-<x_{0 i}><g_{j}>
$$

Para este caso la función de correlación $<h_{i}(t) h_{j}(t)>$ está definida por

$$
<h_{i}(t) h_{j}(t)>=K_{i j}^{0}+K_{i j}^{R}(t)+K_{i j}^{S R}(t)
$$


donde $K_{i j}^{0}$ y $K_{i j}^{R}(t)$ ya han sido definidos anteriormente en la Ec. (3.13). Entonces $K_{i j}^{S R}(t)$ lo definimos como

$$
K_{i j}^{S R}(t) \equiv 2 \sum_{k} \int_{0}^{t} \mathrm{e}^{-a s} R_{i k}(s)<x_{j}(0) \xi_{k}(s)>d s
$$

Nuevamente, el primer término $K_{i j}^{0}$ toma en cuenta el efecto de las condiciones iniciales, el segundo término $K_{i j}^{R}(t)$ es la contribución correspondiente de las fluctuaciones causadas por el ruido interno y la tercera expresión $K_{i j}^{S R}(t)$ es precisamente el efecto del acoplamiento del estado inicial del sistema con el ruido.

De nueva cuenta, el primer y el tercer término requieren de la preparación de las condiciones iniciales del sistema para $t<0$. Así que

$$
\dot{\mathbf{x}}_{0}=-a_{0} \mathbf{x}_{0}+\mathbf{W} \mathbf{x}_{0}+\mathbf{z}(t)
$$

siendo $a_{0}>0$ y $\mathbf{z}(t)$ cumple con las mismas propiedades de ruido de color, cuya intensidad escogemos igual a $Q_{i j}$.

La solución de (3.33) está dada por

$$
\mathbf{x}_{0}=\int_{-\infty}^{0} \mathrm{e}^{a_{0} s} \mathbf{R}(s) \mathbf{z}(s) d^{\prime} s
$$

De manera que el promedio $\left\langle x_{i}(0)>=0\right.$ y su función de correlación $K_{i j}^{0}$ satisface

$$
K_{i j}^{0}=\frac{2}{\tau} \sum_{k} Q_{k k} \int_{-\infty}^{0} \mathrm{e}^{-\left(a_{0}+\tau^{-1}\right) s} R_{i k}(s) d s \int_{-\infty}^{s^{\prime}} \mathrm{e}^{-\left(a_{0}-\tau^{-1}\right) s^{\prime}} R_{j k}\left(s^{\prime}\right) d s^{\prime},
$$

y por tanto $K_{i j}^{S R}(t)$ se transforma en

$$
K_{i j}^{S R}(t)=\frac{2}{\tau} \sum_{k} Q_{k k} \int_{0}^{t} \mathrm{e}^{-\left(a+\tau^{-1}\right) s} R_{i k}(s) d s \int_{-\infty}^{0} \mathrm{e}^{\left(a_{0}+\tau^{-1}\right) s^{\prime}} R_{j k}\left(s^{\prime}\right) d s^{\prime} .
$$

Por otro lado $K_{i j}^{R}(t)$ también se transforma en

$$
K_{i j}^{R}(t)=\frac{2}{\tau} \sum_{k} Q_{k k} \int_{0}^{t} \mathrm{e}^{-\left(a+\tau^{-1}\right) s} R_{i k}(s) d s \int_{0}^{s^{\prime}} \mathrm{e}^{-\left(a-\tau^{-1}\right) s^{\prime}} R_{j k}\left(s^{\prime}\right) d s^{\prime} .
$$

Como podemos observar de (3.35), (3.36) y (3.37), a diferencia de lo que ocurre en el caso de ruido blanco, los elementos de la matriz de rotación están evaluados a tiempos distintos por lo que no podemos evaluar la suma en dichas integrales. De manera que las integrales podrán ser evaluadas en casos particulares de la dinámica de (3.1). 
Si suponemos que los elmentos de matriz $Q_{k k}=Q$ y definimos $\lambda \equiv \frac{2 Q}{\tau} ;$ la Ec. (3.31) toma la siguiente forma

$$
<h_{i}(t) h_{j}(t)>=\lambda\left[\Lambda_{i j}^{0}+\Lambda_{i j}^{R}(t)+\Lambda_{i j}^{S R}(t)\right]
$$

donde

$$
\begin{gathered}
\Lambda_{i j}^{0} \equiv \sum_{k} \int_{-\infty}^{0} \mathrm{e}^{-\left(a_{0}+\tau^{-1}\right) s} R_{i k}(s) d s \int_{-\infty}^{s^{\prime}} \mathrm{e}^{-\left(a_{0}-\tau^{-1}\right) s^{\prime}} R_{j k}\left(s^{\prime}\right) d s^{\prime}, \\
\Lambda_{i j}^{R}(t) \equiv \sum_{k} \int_{0}^{t} \mathrm{e}^{-\left(a+\tau^{-1}\right) s} R_{i k}(s) d s \int_{0}^{s^{\prime}} \mathrm{e}^{-\left(a-\tau^{-1}\right) s^{\prime}} R_{j k}\left(s^{\prime}\right) d s^{\prime}, \\
\Lambda_{i j}^{S R}(t) \equiv \sum_{k} \int_{0}^{t} \mathrm{e}^{-\left(a+\tau^{-1}\right) s} R_{i k}(s) d s \int_{-\infty}^{0} \mathrm{e}^{\left(a_{0}+\tau^{-1}\right) s^{\prime}} R_{j k}\left(s^{\prime}\right) d s^{\prime} .
\end{gathered}
$$

De estas expresiones podemos mostrar que en el límite de tiempos largos, dichas cantidades tienden a sus valores constantes, es decir $\Lambda_{i j}^{R}(\infty)=\Lambda_{i j}^{R}$ y $\Lambda_{i j}^{S R}(\infty)=\Lambda_{i j}^{S R}$ y con ayuda de (3.34) se muestra que $\left\langle h_{i}(t)\right\rangle=0$. Por lo tanto la matriz $\sigma_{i j}$ es igual a

$$
\sigma_{i j}=\sigma_{i j}^{0}+\sigma_{i j}^{R}+\sigma_{i j}^{S R}=\lambda\left(\Lambda_{i j}^{0}+\Lambda_{i j}^{R}+\Lambda_{i j}^{S R}\right)
$$

de los cuales no podemos concluir que satisfacen la propiedad de simetría requerida para poder calcular la densidad de probabilidad conjunta de las variables $h_{i}$ dada por (3.9). Debido a este hecho, no podemos entonces afirmar en general, que el conjunto de variables $h_{i}$ están descorrelacionadas.

En estas circunstancias, para poder continuar con el esquema de TCD alguna hipótesis sobre la matriz $\sigma_{i j}$ debemos de establecer. Dicha hipótesis consiste entonces en suponer que esta matriz $\sigma_{i j}$ de (3.40) sea diagonal con elementos iguales a $\sigma_{i i}=\sigma^{2}$. Esto requerimiento implica evidentemente que cada una de las matrices de (3.40) también deben ser diagonales. Si definimos los elementos de la diagonal de cada matriz de (3.40) como $\sigma_{i i}^{0} \equiv \sigma_{0}^{2}$, de la matriz $\sigma_{i i}^{R} \equiv \sigma_{R}^{2}$ y de $\sigma_{i i}^{S R} \equiv \sigma_{S R}^{2}$, entonces el conjunto de variables $x_{i}(0)$ y $h_{i}$ serán independientes y sus densidades de probabilidad conjuntas estarán dadas por las mismas expresiones (3.18) y (3.22), respectivamente.

Apliquemos ahora el formalismo previo al estudio de los sistemas rotacionales de dos y tres variables, los cuales nos permitirán exhibir con más detalle la descripción teórica anterior. Los resultados obtenidos serán comparados también con los resultados de la formulación estándar del Cap. 2. 


\subsection{Sistemas rotacionales de dos variables}

Para este caso, la matriz $\mathbf{W}$ y la matriz de rotación asociada $\mathbf{R}(t)$ están dadas por

$$
\mathbf{W}=\left(\begin{array}{cc}
0 & \omega \\
-\omega & 0
\end{array}\right), \quad \mathbf{R}(t)=\left(\begin{array}{cc}
\cos \omega t & -\sin \omega t \\
\sin \omega t & \cos \omega t
\end{array}\right) .
$$

Haciendo uso de la forma explícita de la matriz $\mathbf{W}$ podemos demostrar que el rotacional de la fuerza no conservativa es distinto de cero, es decir $\nabla \times \mathbf{W} \mathbf{x}=\vec{\omega} \times \mathbf{x}=-2 \omega \hat{\mathbf{k}}$. En la Fig. 3.1, mostramos una trayectoria estocástica del sistema lineal de dos variables en el plano $\left(x_{1}, x_{2}\right)$, el cual corresponde a una trayectoria espiral hasta alcanzar el valor de referencia $R=1.0$.

De acuerdo con esto, podemos calcular cada uno de los términos de la Ec. (3.40) en el límite de tiempos largos. Para los elementos de matriz $\sigma_{i j}^{0}$ se puede mostrar que, los elementos de la diagonal de dicha matriz son iguales, e.d. $\sigma_{11}^{0}=\sigma_{22}^{0} \equiv \sigma_{0}^{2} \mathrm{y}$

$$
\sigma_{0}^{2}=\frac{Q}{a_{0}\left(1+a_{0} \tau\right)}\left[1-\frac{(\omega \tau)^{2}}{\left(1+a_{0} \tau\right)^{2}+(\omega \tau)^{2}}\right]
$$

Para los elementos fuera de la diagonal se tiene que $\sigma_{12}^{0}=-\sigma_{21}^{0}$, tal que

$$
\sigma_{12}^{0}=\frac{(\omega \tau) Q}{\left(1+a_{0} \tau\right)^{2}+(\omega \tau)^{2}}
$$

De igual forma, los elementos de la diagonal de la matriz $\sigma_{i j}^{R}$ son iguales $\sigma_{11}^{R}=\sigma_{22}^{R} \equiv \sigma_{R}^{2}$ y están dados por

$$
\sigma_{R}^{2}=\frac{Q}{a(1+a \tau)}\left[1-\frac{(\omega \tau)^{2}}{(1+a \tau)^{2}+(\omega \tau)^{2}}\right]
$$

y para los elementos fuera de la diagonal obtenemos que $\sigma_{12}^{R}=-\sigma_{21}^{R}$ donde

$$
\sigma_{12}^{R}=\frac{(\omega \tau) Q}{(1+a \tau)^{2}+(\omega \tau)^{2}}
$$

Para la matriz de acoplamiento $\sigma_{i j}^{S R}$, también se puede mostrar que $\sigma_{11}^{S R}=\sigma_{22}^{S R} \equiv \sigma_{S R}^{2}$ siendo

$$
\sigma_{S R}^{2}=\frac{2 Q \tau}{(1+a \tau)\left(1+a_{0} \tau\right)}\left[1-\frac{(\omega \tau)^{2} S(\omega, \tau)}{\left[\left(1+a_{0} \tau\right)^{2}+(\omega \tau)^{2}\right]\left[(1+a \tau)^{2}+(\omega \tau)^{2}\right]}\right],
$$

donde $S(\omega, \tau)=\left(1+a_{0} \tau\right)^{2}+(1+a \tau)^{2}+\left(1+a_{0} \tau\right)(1+a \tau)+(\omega \tau)^{2}$. Los elementos fuera de la diagonal satisfacen que $\sigma_{12}^{S R}=-\sigma_{21}^{S R}$ siendo

$$
\sigma_{12}^{\mathcal{S R}}=\frac{(\omega \tau) Q}{\left[\left(1+a_{0} \tau\right)^{2}+(\omega \tau)^{2}\right]\left[(1+a \tau)^{2}+(\omega \tau)^{2}\right]} .
$$




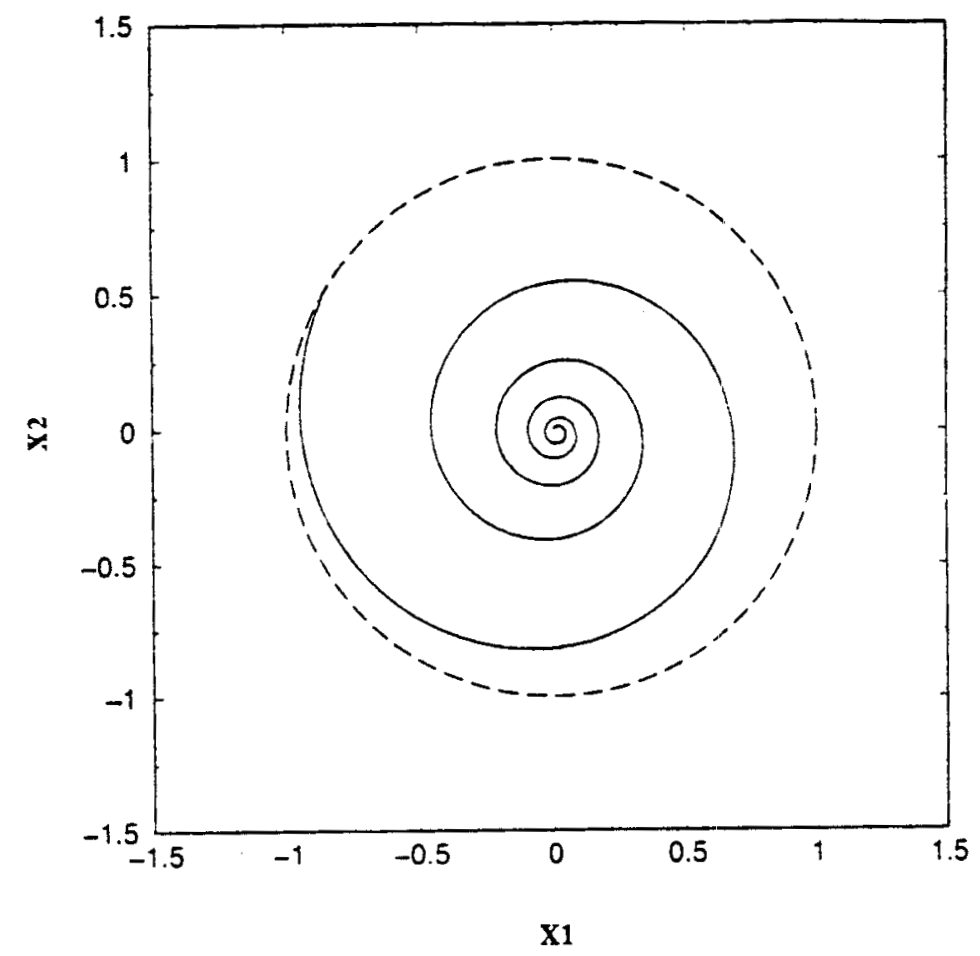

Fig. 3.1: Trayectorias estocásticas del sistema lineal de dos variables

Hagamos ahora un análisis de los resultados obtenidos anteriormente. Primeramente notemos que si $\omega=0$ la matriz $\sigma_{i j}$ es perfectamente una matriz diagonal, puesto que los elementos fuera de la diagonal de las matrices $\sigma_{i j}^{0}, \sigma_{i j}^{R}$ y $\sigma_{i j}^{S R}$ son extrictamente cero y por lo tanto los elementos de la diagonal de la matriz $\sigma_{i j}$ definido por $\sigma^{2}$ deben ser

$$
\sigma^{2}=\sigma_{0}^{2}+\sigma_{R}^{2}+\sigma_{S R}^{2}
$$

el cual coincide con el resultado (2.43) obtenido en la formulación estándar. Es decir, en ausencia de rotaciones los resulatdos son consistentes con la formulación estándar, lo cual era de esperarse.

Si la frecuencia de rotación $\omega \neq 0$, podemos observar en las Ecs. (3.42)-(3.47), la contribución adicional del acoplamiento entre éste parámetro $\omega$ y el tiempo de correlación $\tau$ del ruido. Claramente esta contribución, rompe con la estructura simétrica de las matrices $\sigma_{i j}^{0}, \sigma_{i j}^{R}, \sigma_{i j}^{S R}$ y por lo consiguiente de la matriz $\sigma_{i j}$. Lo cierto de este efecto acoplado, es que su contribución es de forma cuadrática en la mayoría de las ecuaciones anteriores y aparece de forma lineal en el numerador de (3.43), (3.45) y (3.47). Sin embargo, si consideramos la aproximación a primer orden en el tiempo de correlación $\tau \mathrm{y}$, puesto que la intensidad del ruido $Q$ es también pequeño, entonces los términos cuadráticos en las expresiones anteriores desaparecen y las expresiones (3.43), (3.45) y (3.47) pueden ser también despreciados. En este caso la matriz $\sigma_{i j}$ es diagonal $y$ sus 
elementos coinciden nuevamente con los resultados del Cap. 2. Lo anterior significan que en el límite de tiempos largos, el acoplamiento entre $\omega$ y $\tau$ es prácticamente despreciable al inicio del proceso de decaimiento del sistema. En estas circunstancias las escalas de tiempo a primer orden de aproximación en el tiempo de correlación $\tau$ serán las mismas que las obtenidas en el Cap. 2, es decir

(i) Condiciones iniciales fijas

$$
<t_{i}>_{f}=<t_{i}(\tau=0)>_{f}+\frac{\tau}{2}+\mathcal{O}\left(\tau^{2}, Q\right),
$$

donde $<t_{i}(\tau=0)>_{f}$ es igual a (2.45).

(ii) Condiciones inicilaes distribuidas. Caso desacoplado

$$
<t_{i}>_{d}=<t_{i}(\tau=0)>_{d}+\frac{a_{0} \tau}{a_{0}+a}+\mathcal{O}\left(\tau^{2}, Q\right),
$$

con $<t_{i}(\tau=0)>_{d}$ dada por $(2.47)$.

(iii) Condiciones inicilaes distribuidas. Caso acoplado

Para este caso, el tiempo de paso $\left\langle t_{i}\right\rangle_{a}$ es igual a la expresión (2.48) del capítulo anterior.

Para $T_{L}$ y $T_{N L}$ se obtienen siguiendo los mismos pasos del Cap. 2.

\subsection{Sistemas rotacionales de tres variables}

De acuerdo con el Apéndice A.3, los sistemas rotacionales de tres variables muestran un comportamiento similar al caso de dos variables, e.d. las matrices antisimétrica y de rotación están dadas por

$$
\mathbf{W}=\left(\begin{array}{ccc}
0 & \omega & 0 \\
-\omega & 0 & 0 \\
0 & 0 & 0
\end{array}\right), \quad \mathbf{R}(t)=\left(\begin{array}{ccc}
\cos \omega t & -\sin \omega t & 0 \\
\sin \omega t & \cos \omega t & 0 \\
0 & 0 & 1
\end{array}\right)
$$

donde $\omega^{2}=\omega_{1}^{2}+\omega_{2}^{2}+\omega_{3}^{2}$. Nuevamente, podemos demostrar que el rotacional de la fuerza no conservativa es distinto de cero, es decir $\nabla \times \mathbf{W} \mathbf{x}=\vec{\omega} \times \mathbf{x}=-2 \omega \hat{\mathbf{k}}$. En la Fig. 3.2 , mostramos una trayectoria estocástica del sistema lineal de tres variables en el plano $\left(x_{1}, x_{2}, x_{3}\right)$, el cual corresponde a una trayectoria espiral creciente a lo largo del eje $x_{3}$. 
Las expresiones para cada una de las matrices de (3.40) tendrán las siguientes expresiones. Para la matriz $\sigma_{i j}^{0}$ se tiene que $\sigma_{11}^{0}=\sigma_{22}^{0}$, tal que

$$
\sigma_{11}^{2}=\frac{Q}{a_{0}\left(1+a_{0} \tau\right)}\left[1-\frac{(\omega \tau)^{2}}{\left(1+a_{0} \tau\right)^{2}+(\omega \tau)^{2}}\right]
$$

pero $\sigma_{33}^{0}$ está dada por

$$
\sigma_{33}^{0}=\frac{Q}{a_{0}\left(1+a_{0} \tau\right)}
$$

Para los elementos fuera de la diagonal se muestra que $\sigma_{12}^{0}=-\sigma_{21}^{0}$ donde

$$
\sigma_{12}^{0}=\frac{(\omega \tau) Q}{\left(1+a_{0} \tau\right)^{2}+(\omega \tau)^{2}}
$$

$\operatorname{con} \sigma_{13}^{0}=\sigma_{31}^{0}=0$ y $\sigma_{23}^{0}=\sigma_{32}^{0}=0$

Análogamente se muestra para la matriz $\sigma_{i j}^{R}$ que $\sigma_{11}^{R}=\sigma_{22}^{R}$, donde

$$
\begin{gathered}
\sigma_{11}^{R}=\frac{Q}{a(1+a \tau)}\left[1-\frac{(\omega \tau)^{2}}{(1+a \tau)^{2}+(\omega \tau)^{2}}\right], \\
\sigma_{33}^{R}=\frac{Q}{a(1+a \tau)},
\end{gathered}
$$

y los elementos fuera de la diagonal satisfacen que $\sigma_{12}^{R}=-\sigma_{21}^{R}$ de forma tal que

$$
\sigma_{12}^{R}=\frac{(\omega \tau) Q}{(1+a \tau)^{2}+(\omega \tau)^{2}}
$$

pero también $\sigma_{13}^{R}=\sigma_{31}^{R}=0$ y $\sigma_{23}^{R}=\sigma_{32}^{R}=0$.

Para la matriz de acoplamiento $\sigma_{i j}^{S R}$, también se puede mostrar que $\sigma_{11}^{S R}=\sigma_{22}^{S R}$ tal que

$$
\begin{gathered}
\sigma_{11}^{S R}=\frac{2 Q \tau}{(1+a \tau)\left(1+a_{0} \tau\right)}\left[1-\frac{(\omega \tau)^{2} S(\omega, \tau)}{\left[\left(1+a_{0} \tau\right)^{2}+(\omega \tau)^{2}\right]\left[(1+a \tau)^{2}+(\omega \tau)^{2}\right]}\right], \\
\sigma_{33}^{S R}=\frac{2 Q \tau}{\left(1+a_{0} \tau\right)(1+a \tau)},
\end{gathered}
$$

donde $S(\omega, \tau)=\left(1+a_{0} \tau\right)^{2}+(1+a \tau)^{2}+\left(1+a_{0} \tau\right)(1+a \tau)+(\omega \tau)^{2}$.

Los elementos fuera de la diagonal cumplen que $\sigma_{12}^{S R}=-\sigma_{21}^{S R}$ con

$$
\sigma_{12}^{S R}=\frac{(\omega \tau) Q}{\left[\left(1+a_{0} \tau\right)^{2}+(\omega \tau)^{2}\right]\left[(1+a \tau)^{2}+(\omega \tau)^{2}\right]},
$$

con $\sigma_{13}^{S R}=\sigma_{31}^{S R}=0$ y $\sigma_{23}^{S R}=\sigma_{32}^{S R}=0$. Nuevamente, despreciando los efectos del acoplamiento entre $\omega$ y $\tau$ se muetra que los elementos de la diagonal $\sigma^{2}$, de la matriz $\sigma_{i j}^{2}$, satisface la misma expresión (2.43) del Cap. 2, siempre que $Q=D$. 


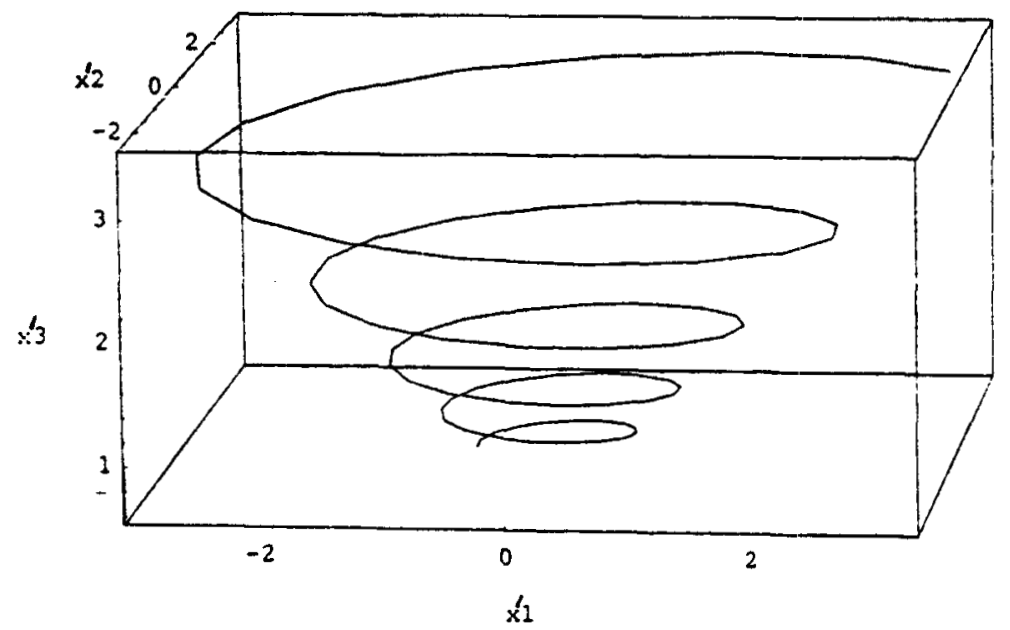

Fig. 3.2: Trayectorias estocásticas del sistema lineal de tres variables

Para estos tres casos estudiados tenemos que los tiempos de paso estarán dadas por las mismas expresiones $(2.44),(2.46)$ y $(2.48)$, respectivamente. $T_{L}$ y $T_{N L}$ se calculan de forma análoga como se hizo en el Cap. 2.

De los resultados obtenidos en las últimas dos secciones, podemos hacer los siguientes comentarios. Hemos visto que en el caso de ruido blanco gaussiano, el formalismo generalizado de TCD se satisface de forma completamente general para cualquier número $n$ de variables, ya que la matriz $\sigma_{i j}$ es perfectamente diagonal. Esto se debe a que en las Ecs. (3.17) y (3.19), el producto de las matrices de rotación están evaluados a tiempos iguales y que además satisfacen la condición de ortogonalidad de dichas matrices. Aparentemente los efectos de rotación del sistema han sido eliminados, pero esto no es así, como veremos un poco más adelante.

En el caso de ruido de color lo anterior ya no es en general válido, ya que de acuerdo con las Ecs. (3.35)-(3.37), el producto de las matrices de rotación están evaluados a tiempos distintos y por lo tanto la condición de ortogonalidad no puede aplicarse de manera inmediata. Esto es consecuencia natural de los efectos de memoria del proceso no markoviano del sistema. Sin embargo, si los efectos del acoplamiento entre el parámetro de rotación $\omega$ y el tiempo de correlación $\tau$ son despreciados al inicio del proceso de decaimiento, entonces a primer orden de aproximación en $\tau$ los requerimientos del formalismo generalizado de TCD se cumplen satisfactoriamente. Nuevamente los efectos rotacionales del sistema han sido aparentemente eliminados. 


\subsection{Justificación del formalismo generalizado de TCD}

El estudio de la caracterización dinámica de los sistemas inestables rotacionales (3.1) mediante el formalismo generalizado de TCD, conduce en el límite de tiempos largos, a las mismas escalas de tiempo obtenidas para caracterizar la dinámica (2.6). Por lo tanto podríamos concluir que dicho formalismo no reporta ninguna contribución novedosa, pues éste se encarga aparenetemente en su descripción de eliminar los efectos de rotación que son inherentes al sistema (3.1).

Pero si esto es así, debemos también 'ser capaces de responder a las siguientes tres preguntas que consideramos son fundamentales para justificar el formalismo generalizado de TCD. Primero, ¿porqué el formalismo generalizado de TCD propuesto para caracterizar la dinámica rotacional (3.1) conduce a las mismas escalas de tiempo de la formulación estándar ?; segundo ¿qué ocurre con los efectos de rotación provenientes de la matriz W? y tercero ¿existirá alguna dinámica equivalente a (3.1) que sea semejante a la dinámica (2.6), para poder entonces justificar que el formalismo generalizado de TCD es consistente en su descripción?

Las respuestas a las tres preguntas anteriores se obtienen haciendo el siguiente análisis. Si en la dinámica (3.1) se realiza el siguiente cambio de variable $\mathbf{y}=\mathrm{e}^{-\mathbf{W} t} \mathbf{x}$, entonces la ecuación de Langevin en el espacio transformado adquiere la siguiente forma

$$
\dot{\mathbf{y}}=a \mathbf{y}+N(r) \mathbf{y}+\mathbf{R}(t) \mathbf{z}(t),
$$

la cual es obviamente equivalente a la dinámica (3.1) y muy semejante a la dinámica (2.6). La función escalar $N(r)$ sigue siendo la misma función que aparece en (3.1), puesto que $r$ es invariante ante dicha transformación, es decir, $r=\mathbf{x}^{T} \mathbf{x}=\mathbf{y}^{T} \mathbf{y}$. En el Apéndice A.1 hemos demostrado que la transformación anterior corresponde precisamente a una matriz de rotación que depende del tiempo, es decir, $\mathbf{R}(t)=\mathrm{e}^{-\mathbf{W} t}$.

Lo que podemos observar de la dinámica (3.61) es ahora lo siguiente. La transformación elimina los efectos de rotación de la fuerza sistemática lineal y los asocia a la fuerza fluctuante; en consecuencia la dinámica (3.61) es bastante similar a (2.6), con la única diferencia de que en la dinámica (3.61) la fuerza fluctuante que definimos como $\mathbf{z}_{R}(t) \equiv \mathbf{R}(t) \mathbf{z}(t)$ es de carácter rotacional. Por otro lado, en el espacio transformado el conjunto de variables y están ahora desacoplados. 


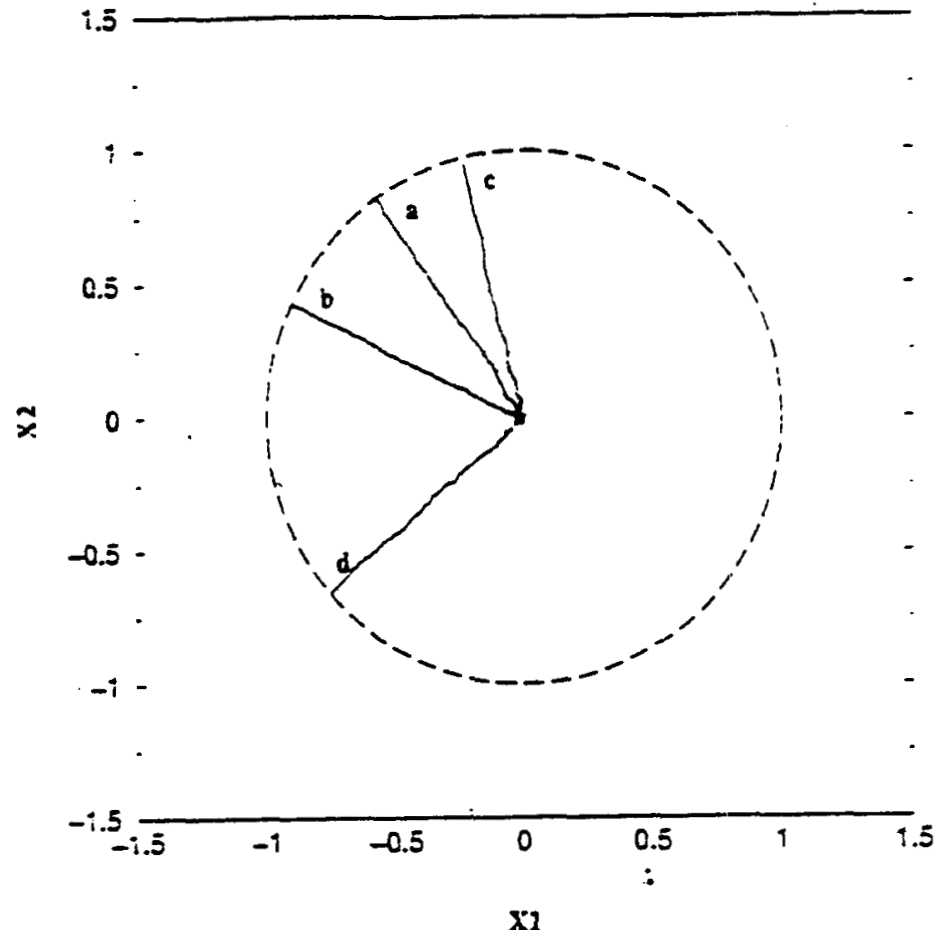

Fig. 3.3: Trayectorias estocásticas para la dinámica lineal de (2.6)

Para visualizar con más claridad la semejanza entre las dinámicas (2.6) y (3.61), tomemos como ejemplo, sin perder la generalidad, a los sistemas rotacionales de dos variables y resolvamos el problema en el régimen lineal.

De acuerdo con los resultados de la simulación numérica, las trayectorias estocásticas descritas por la dinámica lineal de (2.6) en el caso de dos variables, forman un conjunto de líneas rectas que emergen del origen de coordenadas del plano $\left(x_{1}, x_{2}\right)$ hasta alcanzar el valor de referencia $R=1.0$, véase la Fig. 3.3. Las trayectorias emergen en direcciones arbitrarias debido a la estocasticidad de la condición inicial.

Veamos ahora lo que ocurre en el caso de la dinámica lineal de (3.61) para los sistemas de dos variables. Si quitásemos la rotación en el ruido tal que $\mathbf{z}_{R}(i)=\mathbf{z}(t)$, entonces el conjunto de trayectorias estocásticas en el plano $\left(y_{1}, y_{2}\right)$ forman también un conjunto de líneas rectas con un comportamiento bastante semejante o igual a la Fig. 3.3. Si tomamos en cuenta ahora los efectos de rotación del ruido tal que $\mathbf{z}_{R}(t)=\mathbf{R}(t) \mathbf{z}(t)$, entonces dichos efectos hacen que cada línea recta rote alrededor del origen de coordenadas del plano $\left(y_{1}, y_{2}\right)$ en direcciones totalmente aleatorias, véase la Fig. 3.4. Esta situación es análoga para los sistemas de tres variables debido a los resultados del Apéndice A.3.

Dada la descripción anteriror, es obvio entonces inferir que el promedio de los tiempos de paso de cada línea recta en alcanzar el valor de referencia $R=1.0$ de la Fig. 3.4 es el mismo que ocupa cada trayectoria de la Fig. 3.3, pues el conjunto de las líneas 


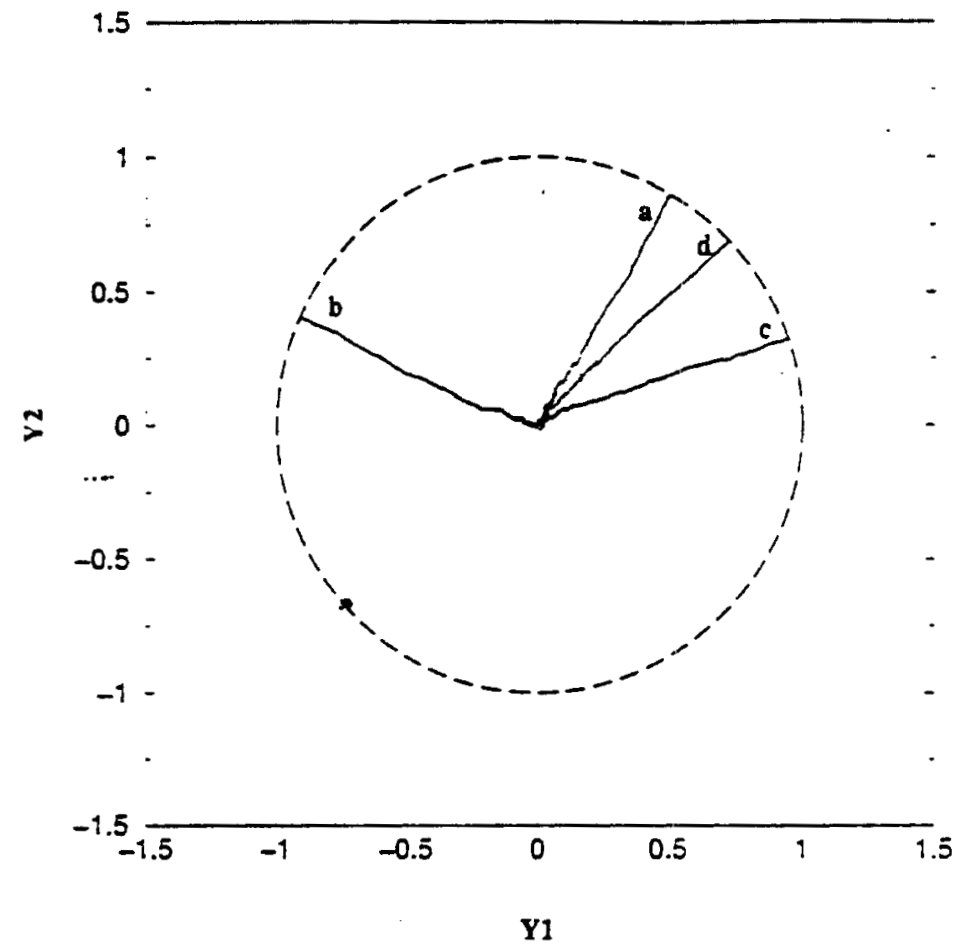

Fig. 3.4: Trayectorias estocásticas para la dinámica lineal de (3.61)

rectas de la dinámica lineal de (3.61) han sido rotadas únicamente alrededor del origen de coordenadas. Por lo tanto podemos concluir que la dinámica de Langevin rotacional (3.1) ha sido transformada en otra dinámica equivalente (3.61) que es también de carácter rotacional, pues en este espacio transformado la rotación ha sido transferida a la fuerza fluctuante.

Por último, si demostramos analíticamente que las escalas de tiempo en el espacio transformado $\mathbf{y}$ de (3.61) son las mismas que en el espacio $\mathbf{x}$ de (3.1), habremos respondido a las tres preguntas formuladas anteriormente.

Para probar esta última afirmación, apliquemos el formalismo generalizado de TCD a la parte lineal de (3.61) descrita por

$$
\dot{\mathbf{y}}=a \mathbf{y}+\mathbf{R}(t) \mathbf{z}(t)
$$

cuya solución es

$$
\mathbf{y}(t)=e^{a t} \mathbf{h}(t)
$$

con $\mathbf{h}(t)$ expresada como

$$
\mathbf{h}(t)=\mathbf{y}(0)+\int_{0}^{t} \mathrm{e}^{-\mathbf{a s}} \mathbf{R}(s) \mathbf{z}(s) d s
$$

donde $\mathbf{y}(0)=\mathbf{x}(0)$. De la misma manera como se hizo en el esquema $\mathbf{x}$ se verifica que en el límite de tiempos largos, la solución (3.63) en términos de la variable $r(t)$, tiene la 
misma forma que (3.7) y (2. 12), es decir

$$
r(t)=\mathbf{y}^{T} \mathbf{y}=h^{2} e^{2 a t}
$$

y por lo consiguiente, el tiempo de paso para alcanzar el valor de referencia $R^{2}$ será

$$
<t_{i}>=\frac{1}{2 a}\left\langle\ln \left(\frac{R^{2}}{h^{2}}\right)\right\rangle
$$

que tiene la misma forma que (3.8) y (2.13); cuya estadística requiere de la densidad de probabilidad marginal $P(h)$. Para ello, observemos que la Ec. (3.64) es exactamente la misma que (3.5) y por lo tanto la densidad de probabilidad conjunta tendrá la misma forma que (3.22) si la matriz $\sigma_{i j}$ satisface las propiedades de simetría requeridas. Finalmente el tiempo de paso (3.66) es el mismo que (3.8) y (2.13).

Para concluir este capítulo, podemos decir que el formalismo generalizado de TCD para caracterizar a la dinámica (3.1), puede visualizarse mucho mejor en el espacio transformado de coordenadas y descrito por la dinámica (3.61), cuyos efectos de rotación están contenidos en la fuerza fluctuante y sus trayectorias son muy semejantes a las de la dinámica (2.6). 


\section{Capítulo 4}

\section{Caracterización dinámica de sistemas inestables rotacionales en presencia de fuerzas externas}

En este cuarto y último capítulo vamos a estudiar la influencia de fuerzas externas constantes o dependientes del tiempo en la relajación dinámica de sistemas inestables rotacionales (3.1) mediante el formalismo de TCD y comparar los resultados con los del capítulo anterior.

En el capítulo anterior hemos mostrado la equivalencia del formalismo de TCD en los dos esquemas dinámicos $\mathbf{x}$ y y; por lo que nuestra descripción en este capítulo se realizará en el espacio transformado de coordenadas, pues es en éste esquema donde el formalismo de TCD es autoconsistente.

Para el espacio x, la dinámica de Langevin (3.1) en presencia de fuerza externa, puede escribirse como

$$
\dot{\mathbf{x}}=a \mathbf{x}+\mathbf{W} \mathbf{x}+N(r) \mathbf{x}+\mathbf{F}_{e}+\mathbf{z}(t)
$$

donde $\mathbf{F}_{e}$ es la fuerza externa representada por el vector columna con elementos $f_{e_{i}}$, los cuales pueden ser constantes o dependientes del tiempo. Nuevamente mediante el cambio de variable $\mathbf{y}=\mathrm{e}^{-\mathbf{W} t} \mathbf{x}$ la ecuación anterior se transforma en

$$
\left.\dot{\mathbf{y}}=a \mathbf{y}+N(r) \mathbf{y}+\mathbf{R}(t)\left[\mathbf{F}_{e}+\mathbf{z}(t)\right)\right]
$$

Como podemos observar la diferencia entre la dinámica (4.2) y (3.61) es obviamente el nuevo término que podemos definir como $\mathbf{F}_{e}^{R} \equiv \mathbf{R}(t) \mathbf{F}_{e}$, el cual representa a la fuerza externa que también es de carácter rotacional. Por lo tanto la presencia de esta fuerza 
externa debe influir en las trayectorias estocásticas del proceso (4.2) produciendo efectos rotacionales en su evolución dinámica. Por ejemplo, si consideramos sólo la parte lineal de (4.2) y en ausencia de fuerza externa, ésta dinámica como ya hemos visto genera trayectorias estocásticas en forma de líneas rectas rotadas aleatoriamente alrededor del origen de coordenadas del espacio y. véase la Fig. 3.3, para el caso particular de dos variables.

Si ahora tomamos en cuenta la presencia de la fuerza externa $\mathbf{F}_{e}^{R}$, éstas trayectorias se verán modificadas por la presencia de dicha fuerza generando así trayectorias rotacionales en su evolución dinámica, los cuales también estarán rotadas aleatoriamente alrededor del origen de coordenadas del espacio y. Esto se mostrará con detalle cuando estudiemos a los sistemas rotacionales de dos y tres variables.

\section{1}

\subsection{Formulación Matricial de la teoría cuasi-deter- minista}

\subsubsection{En presencia de fuerza externa constante}

En este capítulo únicamente vamos a estudiar la caracterización dinámica de (4.2) cuando el ruido interno $\mathbf{z}(t)$ es blanco y gaussiano, cuyas propiedades son tales que su valor medio es nulo y su función de correlación satisface

$$
<\xi_{i}(t) \xi_{j}\left(t^{\prime}\right)>=2 Q_{i j} \delta_{i j} \delta\left(t-t^{\prime}\right), \quad i, j=1, \ldots, n
$$

siendo $Q_{i j}$ la intensidad del ruido.

La caracterización dinámica de (4.2) mediante TCD inicia con la ecuación lineal

$$
\dot{\mathbf{y}}=a \mathbf{y}+\mathbf{R}(t)\left[\mathbf{F}_{e}+\mathbf{z}(t)\right],
$$

cuya solución formal es

$$
\mathbf{y}(t)=\mathrm{e}^{a t} \mathbf{h}(t),
$$

donde

$$
\mathbf{h}(t)=\mathbf{y}(0)+\int_{0}^{t} \mathrm{e}^{-a s} \mathbf{R}(s)\left[\mathbf{F}_{e}+\mathbf{z}(s)\right] d s,
$$

donde $\mathbf{y}(0)$ se refiere a las condiciones iniciales del sistema que supondremos de aquí en adente que son fijas, es decir $\mathbf{y}(0)=0$. 
De forma enteramente similar como se desarrolló el formalismo de TCD en el Cap. 3, podemos garantizar también que para valores pequeños de los elementos de $\mathbf{F}_{e}$ y de $\mathbf{z}(t)$ se satisface la siguiente condición

$$
\lim _{t \rightarrow \infty} \frac{d \mathbf{h}(t)}{d t}=\lim _{t \rightarrow \infty} \mathrm{e}^{-a t} \mathbf{R}(t)\left[\mathbf{F}_{e}+\mathbf{z}(t)\right] \rightarrow 0 .
$$

Por lo tanto en el límite de tiempos largos, $\mathbf{h}(\infty)=\mathbf{h}$ es un vector constante cuyos elementos $h_{i}$ son también variables aleatorias gaussianas. En este régimen de tiempos y en términos de la variable $r$, el proceso (4.5) se convierte en un proceso cuasi-determinista, dado por

$$
r(t)=\mathbf{y}^{T} \mathbf{y}=h^{2} \mathrm{e}^{2 a t},
$$

donde nuevamente $h^{2} \equiv \mathbf{h}^{T} \mathbf{h}=h_{1}^{2}+\cdots+h_{n}^{2}$. El tiempo de paso para alcanzar el valor de referencia $R^{2}$ es de nueva cuenta

$$
<t_{i}>=\frac{1}{2 a}\left\langle\ln \left(\frac{R^{2}}{h^{2}}\right)\right\rangle .
$$

La densidad de probabilidad conjunta de las variables $h_{i}$ satisface también que

$$
P\left(h_{1}, \ldots, h_{n}\right)=\text { cte } \exp \left[-\frac{1}{2} \sum_{i, j}\left(\sigma^{-1}\right)_{i j}\left(h_{i}-<h_{i}>\right)\left(h_{j}-<h_{j}>\right)\right],
$$

donde cte $\equiv \frac{1}{(2 \pi)^{n / 2}\left(\text { Det } \sigma_{i j}\right)^{1 / 2}}$. La matriz $\sigma_{i j}$ debe satisfacer las propiedades de simetría expuestas ya en capítulo anterior. En este caso, el promedio de $h_{i}(t)$ es diferente de cero e igual a

$$
<h_{i}(t)>=\sum_{k} \int_{0}^{t} \mathrm{e}^{-a s} R_{i k}(s) f_{e k} d s .
$$

De acuerdo con (4.6) la función de correlación, está dada por

$$
<h_{i}(t) h_{j}(t)>=K_{i j}^{e}(t)+K_{i j}^{R}(t)
$$

donde el primer término del lado derecho se define como

$$
K_{i j}^{e}(t) \equiv \sum_{k, m} \int_{0}^{t} \int_{0}^{t} \mathrm{e}^{-a\left(s+s^{\prime}\right)} R_{i k}(s) R_{j m}\left(s^{\prime}\right) f_{e k} f_{e m} d s d s^{\prime} .
$$

El segundo término de la expresión (4.12) es el mismo que (3.14) e igual a

$$
K_{i j}^{R}(t) \equiv \sum_{k, m} \int_{0}^{t} \int_{0}^{t} \mathrm{e}^{-a\left(s+s^{\prime}\right)} R_{i k}(s) R_{j m}\left(s^{\prime}\right)<\xi_{k}(s) \xi_{m}\left(s^{\prime}\right)>d s d s^{\prime} .
$$


Con ayuda de las expresiones (4.3) y (4.14) se muestra que

$$
<h_{i}(t) h_{j}(t)>=<h_{i}(t)><h_{j}(t)>+\frac{Q}{a}\left(1-\mathrm{e}^{-2 a t}\right) \delta_{i j} .
$$

Por lo tanto en el límite de tiempos largos, se tiene que la matriz $\sigma_{i j}=\frac{Q}{a} \delta_{i j}$ y por lo consiguiente es una matriz diagonal, cuyos elementos se definen como $\sigma_{i i} \equiv \sigma^{2}$, tal que

$$
\sigma^{2}=\frac{Q}{a}
$$

que es igual a (3.19), pero sin el término $\frac{Q}{a_{0}}$ el cual proviene de condiciones iniciales distribuidas.

Bajo estas condiciones la densidad de probabilidad conjunta (4.10) se reduce a

$$
P\left(h_{1}, \ldots, h_{n}\right)=\frac{1}{\left(2 \pi \sigma^{2}\right)^{n / 2}} \mathrm{e}^{-\alpha^{2}\left[\left(h_{1}-<h_{1}>\right)^{2}+\cdots+\left(h_{n}-<h_{n}>\right)^{2}\right]},
$$

puesto que $<h_{i}>\neq 0$ y donde $\alpha^{2}$ esta definida por $\alpha^{2} \equiv \frac{1}{2 \sigma^{2}}$. La densidad de probabilidad marginal $P(h)$ se obtiene con ayuda de un nuevo espacio de variables, tal que

$$
P\left(h, u_{2}, \ldots, u_{n}\right) d V=\text { cte } \exp \left[-\alpha^{2}\left(h^{2}+q^{2}-2 \mathbf{q}^{T} \mathbf{h}\right)\right] d V,
$$

donde $q^{2} \equiv \mathbf{q}^{T} \mathbf{q}=<h_{1}>^{2}+\cdots+<h_{n}>^{2}$, siendo $\mathbf{q}$ el vector columna de elementos $\left.<h_{i}\right\rangle$. Finalmente $P(h)$ se calcula conociendo el jacobiano de la transformación e integrando sobre el resto de las variables $\left(u_{2}, \ldots, u_{n}\right)$.

\subsubsection{En presencia de fuerza externa dependiente del tiempo}

Si la fuerza externa depende del tiempo, podemos tener dos situaciones que pueden ser objeto de estudio. Una sería el caso en que dicha fuerza fuése de carácter determinista y la otra en la que la fuerza fuera de naturaleza estocástica. En este sección estamos interesados en estudiar el decaimiento dinámico de los sistemas inestables roacionales, no sólo bajo la influencia de las fluctuaciones internas, sino también bajo la acción de fuerzas externas que fluctúan en el tiempo.

En este caso el formalismo de TCD inicia nuevamente con la ecuación lineal

$$
\dot{\mathbf{y}}=a \mathbf{y}+\mathbf{R}(t)\left[\mathbf{F}_{e}(t)+\mathbf{z}(t)\right]
$$


cuya solución, con condiciones iniciales fijas, es

$$
\mathbf{y}(t)=\mathrm{e}^{a t} \mathbf{h}(t)
$$

donde

$$
\mathbf{h}(t)=\int_{0}^{t} \mathrm{e}^{-a s} \mathbf{R}(s)\left[\mathbf{F}_{e}(s)+\mathbf{z}(s)\right] d s .
$$

En analogía al caso constante, también podemos establecer que en el límite de tiempos largos $\mathbf{h}(\infty)=\mathbf{h}$ es un vector constante. La metodología que se sigue para obtener los tiempos de paso, así como la densidad de probabilidad marginal son similares al caso constante, con las diferencias de que ahora

$$
<h_{i}(t)>=\sum_{k} \int_{0}^{t} \mathrm{e}^{-a s} R_{i k}(s) f_{e k}(s) d s,
$$

y

$$
K_{i j}^{e}(t) \equiv \sum_{k, m} \int_{0}^{t} \int_{0}^{t} \mathrm{e}^{-a\left(s+s^{\prime}\right)} R_{i k}(s) R_{j m}\left(s^{\prime}\right)<f_{e k}(s) f_{e m}\left(s^{\prime}\right)>d s d s^{\prime} .
$$

En este caso la función de correlación $\left\langle h_{i}(t) h_{j}(t)>\mathrm{y}\right.$ por lo consiguiente la matriz $\sigma_{i j}$ dependerán de las propiedades estadísticas de la fuerza externa fluctuante $\mathbf{F}_{e}(t)$. Un caso interesante será que dichas propiedades sean distintas a las del ruido interno $\mathbf{z}(t)$, el cual se estudiará en una de las secciones siguientes.

En el límite de tiempos largos podemos establecer que $K_{i j}^{e}(\infty)=\Lambda_{i j}^{e}$, por lo tanto

$$
<h_{i} h_{j}>=\Lambda_{i j}^{e}+\frac{Q}{a} \delta_{i j} .
$$

En este caso las propiedades de simetría de la matriz $\sigma_{i j}$ requiere obviamente del cálculo explícito de $\Lambda_{i j}^{e}$. Esto se realizará con más detalle en la sección 4.2.2.

\subsection{Sistemas rotacionales de dos variables}

\subsubsection{En presencia de fuerza externa constante}

Recordemos que en el caso de dos variables la matriz $\mathbf{W}$ y la matriz de rotación asociada $\mathbf{R}(t)$ están dadas por

$$
\mathbf{W}=\left(\begin{array}{cc}
0 & \omega \\
-\omega & 0
\end{array}\right), \quad \mathbf{R}(t)=\left(\begin{array}{cc}
\cos \omega t & -\sin \omega t \\
\sin \omega t & \cos \omega t
\end{array}\right)
$$


Para calcular la densidad de probabilidad marginal requerimos de los siguientes resultados

$$
<h_{1}>=\frac{a f_{e_{1}}}{a^{2}+\omega^{2}}-\frac{\omega f_{e_{2}}}{a^{2}+\omega^{2}}, \quad<h_{2}>=\frac{\omega f_{e_{1}}}{a^{2}+\omega^{2}}+\frac{a f_{e_{2}}}{a^{2}+\omega^{2}} .
$$

Por lo tanto el parámtro $q^{2}$ estará dada por

$$
q^{2}=\frac{\left|\mathbf{F}_{e}\right|^{2}}{a^{2}+\omega^{2}},
$$

donde $\left|\mathbf{F}_{e}\right|^{2}=f_{e_{1}}^{2}+f_{e_{2}}^{2}$ es el módulo al cuadrado de la fuerza externa.

La densidad de probabilidad marginal está dada ahora por

$$
P(h)=2 \alpha^{2} h I_{0}\left(2 \alpha^{2} h q\right) \mathrm{e}^{-\alpha^{2}\left(h^{2}+q^{2}\right)},
$$

donde $\alpha^{2} \equiv \frac{1}{2 \sigma^{2}}=\frac{a}{2 Q}$ e $I_{0}(x)$ es la función modificada de Bessel de orden cero. En estas condiciones podemos mostrar que el tiempo de paso será

$$
<t_{i}>=<t_{i}>_{0}+\frac{1}{2 a} \psi(1)-\frac{\mathrm{e}^{\beta^{2}}}{2 a} \sum_{m=0}^{\infty} \frac{\beta^{2 m}}{m !} \psi(m+1)+\mathcal{O}(Q)+\mathcal{O}\left(q^{2}\right),
$$

donde definimos el parámetro $\beta^{2} \equiv \alpha^{2} q^{2}$ y $<t_{i}>_{0}$ es el correspondiente tiempo de paso en ausencia de fuerza externa, dado por

$$
<t_{i}>_{0}=\frac{1}{2 a}\left\{\ln \left(\alpha^{2} R^{2}\right)-\psi(1)\right\} .
$$

En el Apéndice C.1 mostramos que la serie de (4.29) puede simplificarse en una expresión más simple, de tal forma que

$$
<t_{i}>=<t_{i}>_{0}+\frac{1}{2 a} \sum_{m=1}^{\infty} \frac{(-1)^{m} \beta^{2 m}}{m m !}+\mathcal{O}(Q)+\mathcal{O}\left(q^{2}\right) .
$$

Para el tiempo de "relajación lineal" $T_{L}$ asociado al momento $\left\langle r^{l}(t)>\right.$ se muestra que

$$
T_{L}=T_{L}^{0}+\frac{1}{2 a} \sum_{m=1}^{\infty} \frac{(-1)^{m} \beta^{2 m}}{m m !}+\mathcal{O}(Q)+\mathcal{O}\left(q^{2}\right),
$$

donde $T_{L}^{0}$ representa la escala de tiempo lineal en ausencia de fuerza externa, tal que

$$
T_{L}^{0}=\frac{1}{2 a}\left\{\ln \left(\alpha^{2} r_{s t}\right)-\psi(1)-\frac{1}{l}\right\}
$$

El tiempo de relajación no lineal $T_{N L}$ asociado también al momento $\left\langle r^{l}(t)>\right.$ será

$$
T_{N L}=T_{N L}^{0}+\frac{1}{2 a} \sum_{m=1}^{\infty} \frac{(-1)^{m} \beta^{2 m}}{m m !},
$$




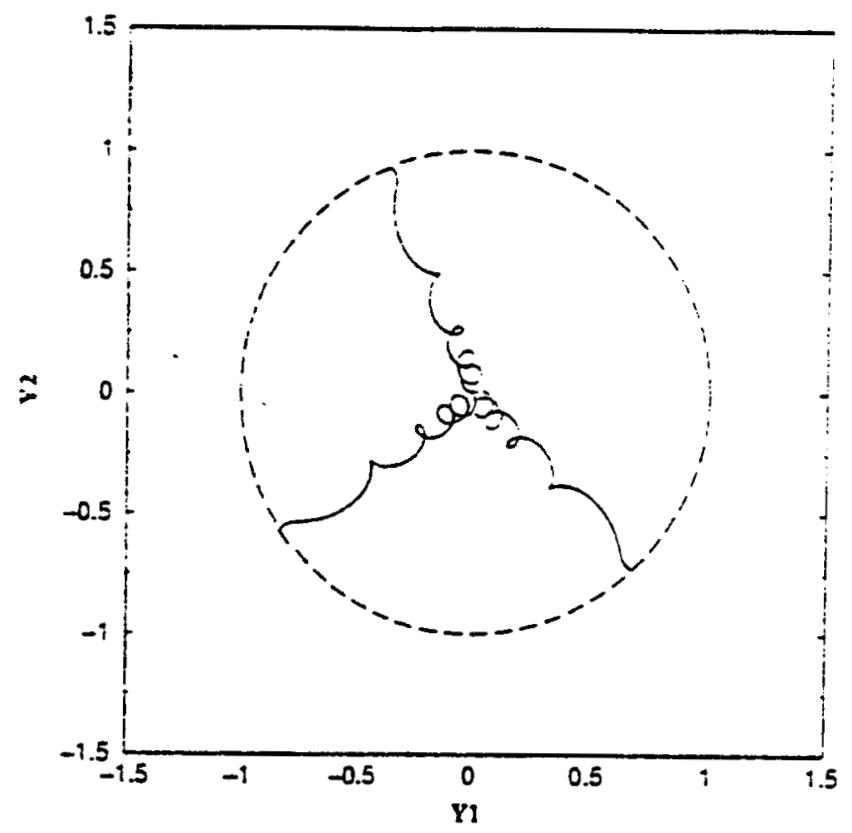

Fig. 4.1: Evolución dinámica de tres trayectorias del sistema de dos variables en el espacio $\left(y_{1}, y_{2}\right)$, para los valores $\left|\mathrm{F}_{e}\right|=1.0, Q=10^{-4}, a=3.0, \omega=6.0$ y $R=1.0$.

siendo $T_{N L}^{0}$ la escala de tiempo de la dinámica no lineal en ausencia de fuerza externa, dada por

$$
T_{N L}^{0}=<t_{i}>_{0}+\mathbf{I}_{N L},
$$

donde $\mathrm{I}_{N L}$ representa las contribuciones no lineales de (4.2) y está dada por (2.58).

Las escalas de tiempo (4.31), (4.32) y (4.35) contienen una serie infinita que es convergente para todo valor de $\beta^{2}=\frac{a\left|F_{e}\right|^{2}}{Q\left(a^{2}+\omega^{2}\right)}$. Como podemos observar, este parámetro es proporcional al cociente $\frac{\left|\mathbb{F}_{e}\right|^{2}}{Q}$, y por lo tanto sugiere analizar dos regiones de aproximación de las escalas de tiempo, a saber $\beta^{2} \leq 1$ ó $\beta^{2}>1$ y verificar en cual de los dos casos se describe de manera precisa el proceso de decaimiento de los sistemas de dos variables. Consideremos únicamente los sistemas lineales ya que para los sistemas no lineales se requiere de la forma explícita de $N(r)$.

Consideremos la región $\beta^{2}>1$, éste corresponde a la situación en la que la amplitud de la fuerza externa domina sobre la intensidad del ruido interno. Para este caso, en la Fig. 4.1 se muestran los resultados de la simulación numérica de algunas de las trayectorias estocásticas que describe la dinámica lineal asociada a (4.2), para escalas de tiempo de observación de acuerdo a la Fig. 4.1 y para los siguientes valores de los parámetros $\left|\mathrm{F}_{e}\right|=1.0, Q=0.0001, a=3.0, \omega=6.0$ y $R=1.0$. En esta situación podemos observar que las trayectorias estocásticas muestran un comportamiento rotacional en forma de 


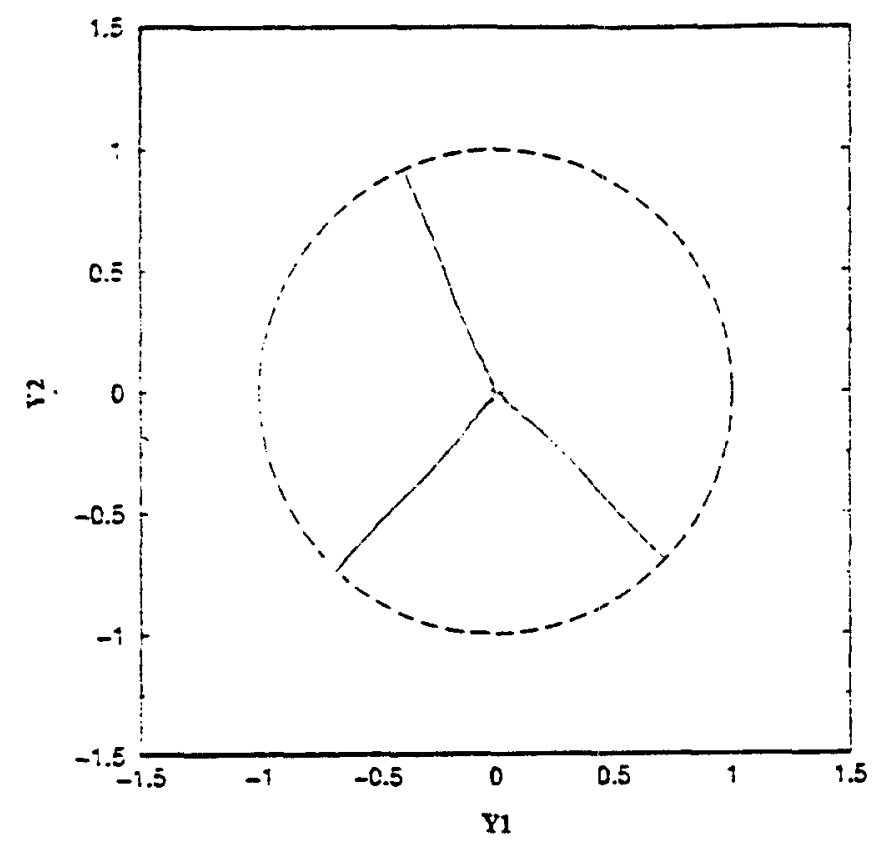

Fig. 4.2: Evolución dinámica de tres trayectorias del sistema de dos variables en el espacio $\left(y_{1}, y_{2}\right)$, para los valores $\left|\mathbf{F}_{e}\right|=Q=10^{-4}, a=3.0, \omega=6.0$ y $R=1.0$.

"bucles", los cuales emergen del origen de coordenadas hasta alcanzar el valor de referencia $R=1.0$, y además rotan de manera aleatoria alrededor del origen de coordenadas. Es importante remarcar que en ausencia de fuerza externa, las trayectorias estocásticas del proceso lineal asociado a (4.2) son líneas rectas como se muestra en la Fig. 3.4; y que la presencia de dicha fuerza externa modifica dichas trayectorias generando trayectorias rotacionales en forma de "bucles" debido precisamente al carácter rotacional de dicha fuerza externa, como se muestra en la Fig. 4.1.

Consideremos ahora el caso de $\beta^{2} \leq 1$, el cual corresponde a la situación en la que la amplitud de la fuerza externa es menor o igual que la intensidad del ruido interno. En la Fig. 4.2, se muestran las mismas trayectorias de la Fig. 4.1, con los mismos valores anteriores de los parámetros, excepto que ahora $\left|\mathrm{F}_{e}\right|=Q=0.0001$, es decir, la amplitud de la fuerza externa iguala la intensidad del ruido interno. Lo que podemos concluir en este caso es que, en esta escala de tiempos largos podemos observar que los efectos de rotación o "bucles" de las trayectorias estocásticas son casi imperceptibles y por lo tanto dichas trayectorias estocásticas se comportan prácticamente como si fueran líneas rectas. En esta escala de tiempo de observación la amplitud de la fuerza externa es tan débil que no podemos apreciar sus efectos. Esto obviamente no es así a escalas de tiempos no tan largos donde los efectos rotacionales son bastante notorios. 


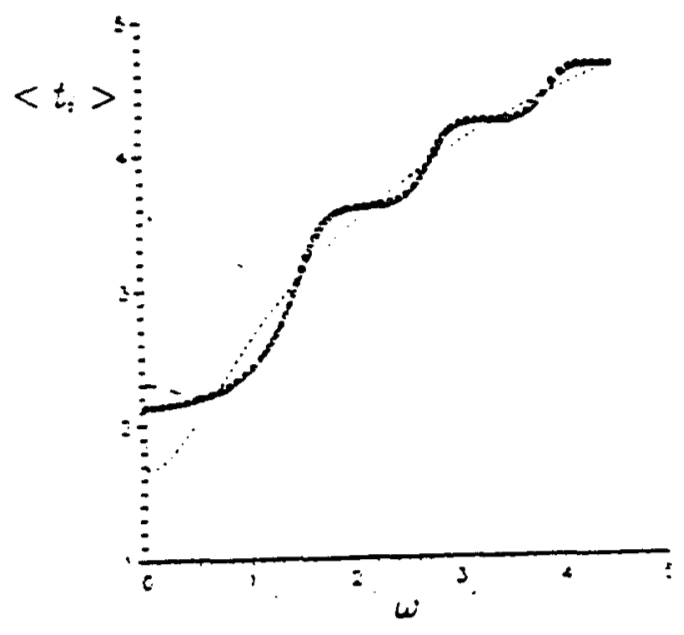

Fig. 4.3: Comparación entre la escala de tiempo (4.36)(línea continua) y la simulación numérica (puntos), para el caso $\left|\mathrm{F}_{e}\right|=1.0, Q=10^{-4}, a=3.0, \omega=6.0$ y $R=1.0$.

La pregunta natural que podemos formular ahora es la siguiente. ¿En cuál de las dos regiones es válida la descripción de TCD? Aunque la respuesta a la pregunta debe corroborarse con resultados de simulación numérica, podemos adelantarnos un poco a la respuesta si tomamos en cuenta los resultados del capítulo anterior, en el que hemos mostrado que TCD es consistente si se desprecian los efectos de la rotación en la trayectoria dinámica del sistema, es decir, TCD debe ser válida en la región de $\beta^{2} \leq 1$.

Véamos ahora lo que ocurre con los resultados teóricos obtenidos para el tiempo de paso en las dos regiones de $\beta^{2}$. Primeramente para la región $\beta^{2}>1$, el tiempo de paso (4.31) puede reducirse, con ayuda de la identidad (C.10) del Apéndice C.1, a la siguiente expresión

$$
<t_{i}>\simeq \frac{1}{2 a} \ln \left(\frac{a^{2}+\omega^{2}}{\left|\mathrm{~F}_{e}\right|^{2}}\right) .
$$

Esta escala de tiempo coincide en el límite determinista de (4.2). Por lo tanto, en la región de aproximación $\beta^{2}>1$ el efecto del ruido es prácticamente despreciable y el término dominante es la expresión (4.36).

En la Fig. 4.3 se muestran los resultados de la simulación numérica (puntos) del tiempo de paso en función del parámetro de rotación de las trayectorias rotacionales de la Fig. 4.1, en la que se observa un comportamiento oscilatorio de dicha escala de tiempo. La línea continua representan el resultado teórico (4.36). En la figura, podemos obser- 


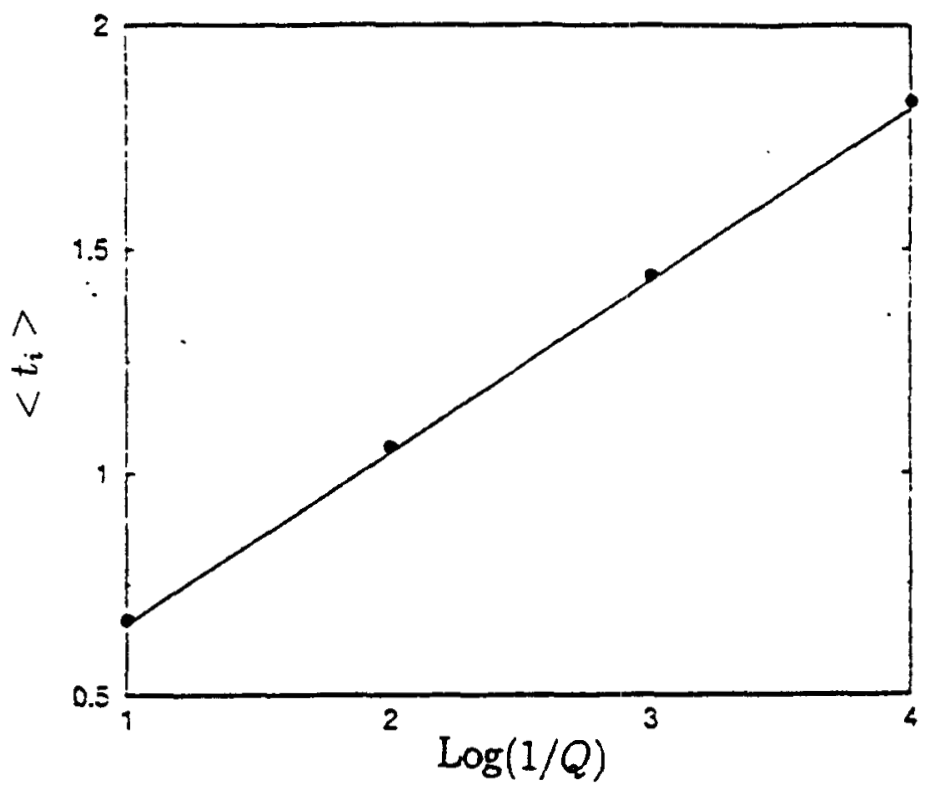

Fig. 4.4: Comparación entre la escala de tiempo (4.31)(línea continua) y la simulación numérica (puntos), para el caso $\left|F_{e}\right|=Q=10^{-4}, a=3.0 . \omega=6.0$ y $R=1.0$. Los resultados de simulación corresponden a $Q=10^{-1}, Q=10^{-2}, Q=10^{-3}, Q=10^{-4}$.

var claramente que dicho resultado teórico no describe correctamente el comportamiento oscilatorio del sistema.

Por otro lado, para la región $\beta^{2} \leq 1$, el término dominante será el primer término de (4.31), es decir $\left\langle t_{i}\right\rangle_{0}$ ya que la contribución de la serie será muy pequeña. En la Fig. 4.4, mostramos los resultados del tiempo de paso promedio (4.31) en función del $\log (1 / Q)$, del conjunto de trayectorias de la Fig. 4.2. La gráfica muestra una línea recta para el resultado teórico (4.31). Los puntos corresponden a los resultados de la simulación numérica del proceso lineal asociado a (4.2). En este caso podemos observar una excelente concordancia entre ambos resultados; en consecuencia la descripción de TCD debe ser válida en la región en la que la amplitud de fuerza externa es igual o menor que la intensidad del ruido interno.

\subsubsection{En presencia de fuerza externa dependiente del tiempo}

Consideremos ahora el caso en el que las componentes de la fuerza externa son funciones armónicas de una fase $\phi(t)$ que fluctúa en el tiempo alrededor de un valor promedio $\phi_{0}=0$, de manera que la primera componente de la fuerza será $f_{e_{1}}=\left|\mathrm{F}_{e}\right| \cos \phi(t)$ y la segunda componente $f_{e_{2}}=\left|\mathrm{F}_{e}\right| \sin \phi(t)$, donde $\left|\mathrm{F}_{\mathrm{e}}\right|$ es la amplitud constante de la 
fuerza externa. Proponemos que la fase fluctuante satisfaga las propiedades del modelo de difusión gaussiano, con valor medio nulo y función de correlación

$$
<\phi(t) \phi\left(t^{\prime}\right)>=2 D \min \left(t, t^{\prime}\right)
$$

donde $D$ es la intensidad de la fase fluctuante. En estas condiciones podemos mostrar que los valores promedio de las componentes $h_{1}$ y $h_{2}$ vienen dadas por las siguientes expresiones

$$
<h_{1}>=\frac{\left|\mathbf{F}_{e}\right|(a+D)}{(a+D)^{2}+\omega^{2}}, \quad<h_{2}>==\frac{\left|\mathbf{F}_{e}\right| \omega}{(a+D)^{2}+\omega^{2}},
$$

de tal forma que el parámetro $q^{2}$ es ahora

$$
q^{2}=\frac{\left|\mathbf{F}_{e}\right|^{2}}{(a+D)^{2}+\omega^{2}},
$$

siendo $\left|\mathrm{F}_{e}\right|^{2}=f_{e_{1}}^{2}+f_{e_{2}}^{2}$. Si comparamos la expresión (4.39) con (4.27) notamos que el efecto de las fluctuaciones de fase produce un corrimiento en el parámetro de rotación, definiendo así un parámetro efectivo $\omega_{e_{f}}^{2}=\omega^{2}+D^{2}+2 a D$.

De acuerdo con (4.23) y (4.24) podemos ahora calcular los elementos de matriz de $\Lambda_{i j}^{e}$, y por lo consiguiente los elementos de la matriz $\sigma_{i j}$, de tal forma que

$$
\begin{aligned}
& \sigma_{11}=\frac{Q}{a}+\frac{\left|\mathbf{F}_{e}\right|^{2}(a+D)}{2 a\left[(a+D)^{2}+\omega^{2}\right]}+\frac{\left|\mathbf{F}_{e}\right|^{2}\left[(a+D)(a+2 D)-\omega^{2}\right]}{2\left[(a+D)^{2}+\omega^{2}\right]\left[(a+2 D)^{2}+\omega^{2}\right]}-\frac{\left|\mathbf{F}_{e}\right|^{2}(a+D)^{2}}{\left[(a+D)^{2}+\omega^{2}\right]^{2}}, \\
& \sigma_{22}=\frac{Q}{a}+\frac{\left|\mathbf{F}_{e}\right|^{2}(a+D)}{2 a\left[(a+D)^{2}+\omega^{2}\right]}-\frac{\left|\mathbf{F}_{e}\right|^{2}\left[(a+D)(a+2 D)-\omega^{2}\right]}{2\left[(a+D)^{2}+\omega^{2}\right]\left[(a+2 D)^{2}+\omega^{2}\right]}-\frac{\left|\mathbf{F}_{e}\right|^{2} \omega^{2}}{\left[(a+D)^{2}+\omega^{2}\right]^{2}}, \\
& \sigma_{12}=\sigma_{21}=\frac{\left|\mathbf{F}_{e}\right|^{2}(2 a+3 D) \omega}{2\left[(a+D)^{2}+\omega^{2}\right]\left[(a+2 D)^{2}+\omega^{2}\right]}-\frac{\left|\mathbf{F}_{e}\right|^{2} \omega}{2 a\left[(a+D)^{2}+\omega^{2}\right]}-\frac{\left|\mathbf{F}_{e}\right|^{2} \omega(a+D)}{\left[(a+D)^{2}+\omega^{2}\right]^{2}} .
\end{aligned}
$$

Los resultados obtenidos nos dicen que la matriz $\sigma_{i j}$ no es diagonal y por lo consiguiente no se satisfacen las requerimientos de TCD. Veamos ahora bajo que condiciones estos requerimientos pueden ser satisfechos. Hagamos primeramente una aproximación a primer orden en la intensidad de las fluctuaciones de fase, es decir, consideremos que estas intensidades son muy pequeñas de tal forma que las cantidades arriba calculadas pueden aproximarse a

$$
\sigma_{11}=\frac{Q}{a}+\frac{\left|F_{e}\right|^{2} D}{2 a\left(a^{2}+\omega^{2}\right)}-\frac{a\left|F_{e}\right|^{2}\left(a^{2}-3 \omega^{2}\right) D}{2\left(a^{2}+\omega^{2}\right)^{3}}+\mathcal{O}\left(D^{2}\right),
$$




$$
\begin{gathered}
\sigma_{22}=\frac{Q}{a}+\frac{\left|F_{e}\right|^{2} D}{2 a\left(a^{2}+\omega^{2}\right)}+\frac{a\left|F_{e}\right|^{2}\left(a^{2}-3 \omega^{2}\right) D}{2\left(a^{2}+\omega^{2}\right)^{3}}+\mathcal{O}\left(D^{2}\right), \\
\sigma_{12}=\sigma_{21}=-\frac{\omega\left|F_{e}\right|^{2}\left(a^{2}-3 \omega^{2}\right) D}{2\left(a^{2}+\omega^{2}\right)^{3}}-\frac{\omega\left|F_{e}\right|^{2}}{2 a\left(a^{2}+\omega^{2}\right)}+\mathcal{O}\left(D^{2}\right) .
\end{gathered}
$$

De estas expresiones podemos notar todavía que la matriz $\sigma_{i j}$ no es diagonal, por lo que las variables $\left(h_{1}, h_{2}\right)$ siguen estando correlacionadas. Por otro lado, debemos recordar que TCD es válida si el parámetro $\beta^{2} \leq 1$, de acuerdo con el análisis de la sección anterior. Mostramos también que en dicha región de aproximación, los efectos de rotación deben ser despreciables. Por lo tanto, si queremos satisfacer los requerimientos de TCD entonces debemos considerar esta región de aproximación, añadiendole además la aproximación de pequeñas fluctuaciones de fase. Bajo estas circunstancias podemos concluir que la matriz $\sigma_{i j}$ es diagonal, con elementos $\sigma_{i i}=\sigma^{2}$, tal que

$$
\sigma^{2}=\frac{Q}{a}+\frac{\left|\mathbf{F}_{e}\right|^{2} D}{2 a\left(a^{2}+\omega^{2}\right)}+\mathcal{O}\left(D^{2}\right)
$$

Así las escalas de tiempo en este caso, son similares a (4.31), (4.32) y (4.34), con $\beta^{2} \equiv \alpha^{2} q^{2}=\frac{q^{2}}{2 \sigma^{2}}$.

Finalmente, el formalismo de TCD se satisface si la amplitud de la fuerza externa que fluctúa a través de su fase, es menor o igual que la intensidad del ruido interno y además si la intensidad de la fase es pequeña.

Veamos ahora como la teoría desarrollada en este capítulo puede aplicarse para detectar señales ópticas débiles en un sistema Láser mediante el conocimiento de los tiempos de encendido de dicho sistema. Para ello hagamos una breve introducción acerca del sistema Láser y cómo este sistema admite una descripción en el esquema de Langevin.

\subsection{Sistema Láser}

El decaimiento de estados inestables es un proceso típico en el que las fluctuaciones estocásticas juegan un papel muy importante. El proceso de encendido de un Láser es un ejemplo de este tipo de procesos dinámicos, el cual puede ser caracterizado a través de las propiedades estadísticas de los tiempos de inicialización de dicho proceso $[44,50,64$. $59,2]$. La presencia inevitable del ruido interno (ruido de emisión expontánea) causa ‘ decaimiento del estado inestable inicial, desde el valor inicial $I(0)$ de la initensidad hasta 


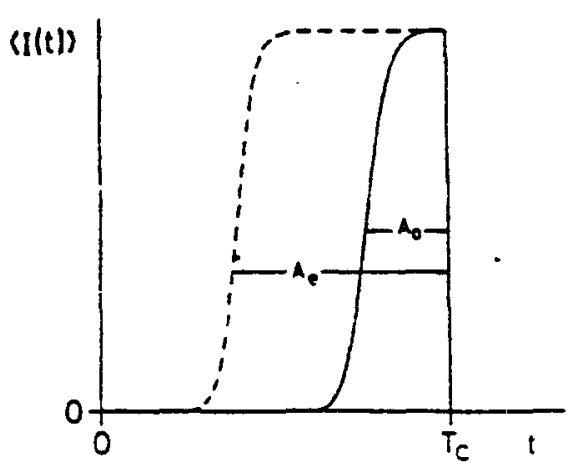

Fig. 4.5: Evolución dinámica de $\langle I(t)\rangle$ durante el intervalo de tiempo $T_{c}$. $A_{0}$ es la área bajo la curva sólida $\left(E_{e}=0\right) . A_{e}$ es el área bajo la curva puntiada $\left(E_{e} \neq 0\right)$

un valor final de referencia preestablecido $I_{r}$, el cual se considera en un cierto porcentaje del valor del estado estacionario $I_{s t}$. La presencia de señales externas débiles también afecta este tiempo de iniciación, acelerando el proceso de decaimiento del sistema.

A finales de los ochenta y principios de los noventa del siglo pasado, Vemuri y Roy [61], Littler et. al. [36] mostraron desde un punto de vista numérico y experimental respectivemente, que las señales ópticas débiles de un Láser pueden ser detectados usando a otro sistema Láser como un receptor super-regenerativo. Las señales débiles detectadas son menores o del mismo orden de magnitud que la intensidad del ruido interno, pero $10^{8}$ veces menor que la intensidad del estado estacionario. Un receptor super-regenarativo opera de tal forma que una señal externa débil incidente es amplificada en un oscilador que es periódicamente encendido y apagado.

Los resultados numéricos y experimentales han sido sustentados a través de los tiempos de paso [2] y los tiempos de relajación no lineales [20]. En esta sección estudiaremos el método propuesto en la Ref. [20] para detectar las señales ópticas débiles en un Láser, el cual consiste en calcular una cantidad llamada receptor de salida que se define como $S \equiv \frac{A_{e}}{A_{0}}$, donde $A_{e}$ es el área bajo la curva de la evolución promedio de la intensidad $\left\langle I(t)>\right.$ del campo eléctrico en presencia de la señal externa y $A_{0}$ es el área bajo la curva en ausencia de dicha señal, ver Fig. 4.5. Esta cantidad está en función de los tiempos de relajación no lineales.

Por otro lado, la obtención de una ecuación determinista que describa la fenomenología del sistema Láser se obtiene a partir de las ecuaciones que describen la interacción radiación-materia. Estas ecuaciones microscópicas se obtienen a partir de principios 
cuánticos, pero dadas las diferentes escalas de evolución de las variables involucradas, principalmente el campo eléctrico transmitido y las variables atómicas, resulta ser que el campo eléctrico evoluciona mucho más lentamente con lo cual las variables atómicas pueden eliminarse adiabáticamente. Como resultado final se obtiene una ecuación que describe el proceso de evolución del Láser, que contiene todos los parámetros macroscópicos que se observan. El campo eléctrico que es una magnitud compleja, tendrá el papel de variable relevante. El interés estará principalmente en conocer la intensidad del campo eléctrico aunque muchas veces habrá que estudiar también la fase del mismo.

La introducción de las fluctuaciones en la ecuaciones fenomenológicas y deterministas del Láser se hace mediante dos mecanismos siguiendo los pasos habituales en la literatura [18]. En primer lugar hemos de dar entrada a las fluctuaciones de origen cuántico que se despreciaron en el proceso de eliminación de las variables atómicas y que son necesarias para entender por ejemplo el procesode emisión espontánea. Estas fluctuaciones se tienen en cuenta mediante la introducción de ruidos aditivos gaussianos (blanco) en las ecuaciones deterministas. Los valores de los parámetros de estos ruidos se asignan a partir de los resultados experimentales. Este tipo de fluctuaciones se suelen llamar internas en la literatura, puesto que son propias del sistema. Existen una segunda fuente de fluctuaciones muy importante que son de origen externo. La necesidad de explicar algunos resultados experimentales hace necesario su introducción [33]. Estas fluctuaciones se introducen a través del bombeo óptico, y en ciertos sistemas son controlables en el laborario [45]. Se denominan fluctuaciones externas debido a su origen. Su característica más importante descansa en el hecho de su independencia respecto del sistema, de ahí su nombre, y por lo tanto han de tener unos valores y una escala de tiempo finitas, lo que conduce de forma natural a cuestionar sus propiedades gaussianas y de ruido blanco [10].

\subsubsection{En presencia de campo externo constante}

Discutidas las generalidades anteriores presentamos el modelo más simple de todos y que corresponde al sistema Láser de un sólo modo y operando en resonancia $[2,36,8]$

$$
\dot{E}=\left(-\gamma_{1}+i \gamma_{2}\right) E+\frac{F}{1+\frac{A}{F} I} E+k_{e} E_{e}+\xi(t)
$$

donde $\gamma_{1}$ es la tasa de decaimiento de la cavidad, $\gamma_{2}$ es el parámetro de desintonización entre el campo Láser y la señal inyectada $E_{e}, F$ es el parámetro de ganancia, $A$ el parámetro 
de saturación, $I=|E|^{2}=E_{1}^{2}+E_{2}^{2}$ es la intensidad del campo del eléctrico complejo, $E_{e}$ es el campo eléctrico externo constante o señal externa y $k_{e}$ es el parámetro de acoplamiento. La emisión espontánea es modelada por la fuerza aleatoria compleja $\xi(t)=\xi_{1}(t)+i \xi_{2}(t)$, con valor medio nulo y función de correlación

$$
<\xi(t) \xi^{*}\left(t^{\prime}\right)>=2 \epsilon \delta\left(t-t^{\prime}\right), \quad 226001
$$

que debe ser compatible con (4.3) si $Q_{i i}=Q=\frac{\epsilon}{2}$. Para calcular el tiempo de relajación no lineal, necesitamos primeramente describir el régimen lineal de (4.47), la cual puede escribirse en forma matricial, como

$$
\left(\begin{array}{c}
\dot{E}_{1} \\
\dot{E}_{2}
\end{array}\right)=\left(\begin{array}{cc}
a & -\gamma_{2} \\
\gamma_{2} & a
\end{array}\right)\left(\begin{array}{c}
E_{1} \\
E_{2}
\end{array}\right)+k_{e}\left(\begin{array}{c}
E_{e_{1}} \\
E_{e_{2}}
\end{array}\right)+\left(\begin{array}{l}
\xi_{1}(t) \\
\xi_{2}(t)
\end{array}\right)
$$

donde $a \equiv\left(F-\gamma_{1}\right)$. Esta ecuación es perfectamente compatible con la descripción teórica de la Secc. 4.2.1, si hacemos que los siguientes parámetros $\gamma_{2}=-\omega, f_{e_{1}}=k_{e} E_{e_{1}}$ y $f_{e_{2}}=k_{e} E_{e_{2}}$. Para este sistema se tiene que

$$
<h_{1}>=\frac{a k_{e} E_{e_{1}}}{a^{2}+\omega^{2}}+\frac{\omega k_{e} E_{e_{2}}}{a^{2}+\omega^{2}}, \quad<h_{2}>=-\frac{\omega k_{e} E_{e_{1}}}{a^{2}+\omega^{2}}+\frac{a k_{e} E_{e_{2}}}{a^{2}+\omega^{2}}
$$

de modo que el parámetro $q^{2}$, está dado por

$$
q^{2}=\frac{k_{e}^{2}\left|E_{e}\right|^{2}}{a^{2}+\omega^{2}}
$$

donde $\left|E_{e}\right|^{2}=E_{e_{1}}^{2}+E_{e_{2}}^{2}$. Puesto que $h$ es un número complejo, la varianza de dicho número se define como

$$
\sigma^{2} \equiv<h h^{*}>-|<h>|^{2}=\sigma_{11}+\sigma_{22}=\frac{2 Q}{a}=\frac{\epsilon}{a}
$$

Para calcular $T_{N L}$ debemos de escribir la ecuación determinista asociada a (4.47) incluyendo las contribuciones no lineales en términos de la intensidad $I$ de la forma (2.1); es decir

$$
\dot{I}=\frac{2 a I\left(I_{s t}-I\right)}{I_{s t}\left(1+\frac{\dot{A}}{F} I\right)}
$$

donde $I_{s t}=\frac{a F}{\gamma_{1} A}$. De acuerdo con esto podemos identificar los términos $C_{0}=\frac{I_{s t}}{2 a} \mathrm{y}$ $g(I)=\frac{A I_{s t}}{2 a F}$. La contribución del término no lineal $I_{N L}$ para el orden del momento $l=1$, puede escribirse en el límite de intensidad de ruido pequeño y de amplitud pequeña del 
campo externo, como $\mathrm{I}_{N L}=\frac{1}{2 a}\left(\frac{F}{\gamma_{1}}-1\right)$. Por lo tanto el tiempo de relajación no lineal asociado al momento $\langle I(t)\rangle$, está dado por

$$
T_{N L}=T_{N L}^{0}+\frac{1}{2 a} \sum_{m=1}^{\infty} \frac{(-1)^{m} \beta^{2 m}}{m m !}+\mathcal{O}(\epsilon)+\mathcal{O}\left(q^{2}\right),
$$

donde

$$
T_{N L}^{0}=\frac{1}{2 a}\left\{\ln \left(\frac{a I_{s t}}{2 \epsilon}\right)-\psi(1)+\frac{F}{\gamma_{1}}-1\right\}+\mathcal{O}(\epsilon)
$$

Estas escalas de tiempo son las mismas que las reportadas en las Refs. [20,23] excepto por el factor $q^{2}$ de (3.51) el cual contiene al parámetro $\omega=\gamma_{2}$. En esas referencias el estudio se realiza a partir de una dinámica de Láser no rotacional.

\subsubsection{Receptor de Salida}

El receptor de salida está dado en función de los tiempos de realjación no lineales definidos de la siguiente manera

$$
T_{N L}=\int_{0}^{\infty} m(t) d t=\frac{1}{M_{0}} \int_{0}^{T_{c}}\left[<r^{l}(t)>-1<r^{l}>_{s t}\right] d t
$$

donde $T_{c}$ es el tiempo durante el cual el sistema relaja desde su estado inestable inicial $I(0) \simeq 0$ hasta un valor muy cercano a su estado estacionario $I_{s t}, \mathrm{y}$ tiene sentido si $T_{c} \geq 1.5 T_{0}$ donde $T_{0}$ es el TRNL en ausencia de campo externo.

Haciendo un poco algebra tenemos que

$$
\begin{aligned}
& T_{e} M_{0}=A_{e}-T_{c}<r^{l}>_{s t}, \\
& T_{0} M_{0}=A_{0}-T_{c}<r^{l}>_{s t},
\end{aligned}
$$

donde $T_{e}$ es el TRNL en presencia de fuerza externa. Si las condicions iniciales son fijas entonces $\left\langle r_{0}^{l}\right\rangle \simeq 0$. En estas condiciones el receptor de salida será

$$
S \equiv \frac{A_{e}}{A_{0}}=\frac{T_{e}-T_{c}}{T_{0}-T_{c}}=1+\frac{T_{0}-T_{e}}{T_{c}-T_{0}} .
$$

De acuerdo con (4.54) se tiene finalmente que

$$
S=1-\frac{1}{2 a\left(T_{c}-T_{0}\right)} \sum_{m=1}^{\infty} \frac{(-1)^{m} \beta^{2 m}}{m m !} .
$$

El receptor de salida es una cantidad sensible a la presencia de sañales débiles, como se puede observar en la Fig. 4.6, que muestra la comparación de los resultados de la simulación numérica con los resultados teóricos de (4.59). 


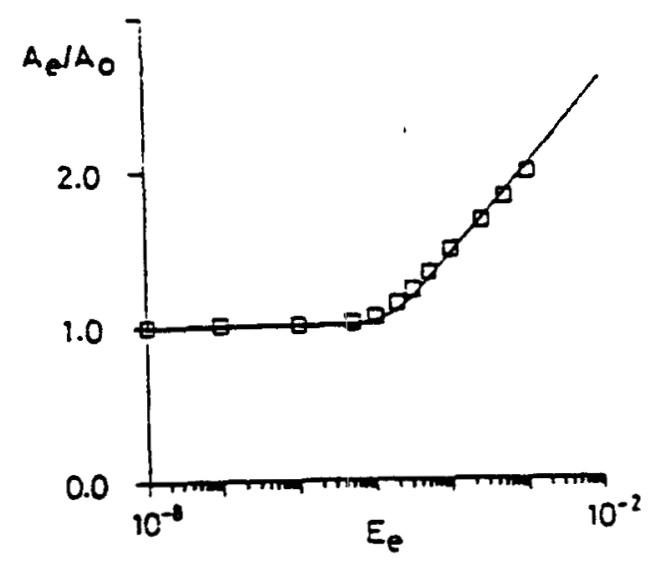

Fig. 4.6: Receptor de salida $\frac{A_{e}}{A_{0}}$ contra el módulo del campo externo $E_{e}$. La línea continua representa los resultados teóricos de la Ref. [20]. Los cuadrados corresponden a los resultados de simulación [61]

\subsubsection{En presencia de campo externo dependiente del tiempo}

El formalismo matricial puede aplicarse al estudio de un sistema Láser como el que se reporta en la Ref. [9], donde se investiga las consecuencias de las fluctuaciones de fase de un campo débil inyectado en el proceso de encendido de un Láser de clase A, a través de la distribución de los tiempos de paso. La ecuación dinámica tipo Langevin que describe al modelo Láser de dicha referencia, se escribe como

$$
\dot{E}=-\left(\gamma_{1}+i \gamma_{2}\right) E+\frac{F}{1+\frac{A}{F} I} E+k_{e}\left|E_{e}\right| \mathrm{e}^{i \phi(t)}+\xi(t),
$$

donde los parámetros del sistema ya han sido definidos en la Subs. 4.3.1, el tercer término $\left|E_{e}\right| e^{i \phi(t)}$ corresponde al campo externo complejo con fase fluctuante y $\left|E_{e}\right|$ es su amplitud constante. El ruido de emisión espontánea es el mismo que (4.48), y es compatible son (4.3) siempre y cuado $Q_{k k}=Q=\frac{\epsilon}{2}$. De nueva cuenta en el régimen lineal la Ec. (4.50) puede escribirse, como

$$
\left(\begin{array}{c}
\dot{E}_{1} \\
\dot{E}_{2}
\end{array}\right)=\left(\begin{array}{cc}
a & \gamma_{2} \\
-\gamma_{2} & a
\end{array}\right)\left(\begin{array}{l}
E_{1} \\
E_{2}
\end{array}\right)+k_{e}\left|E_{e}\right|\left(\begin{array}{c}
\cos \phi(t) \\
\sin \phi(t)
\end{array}\right)+\left(\begin{array}{c}
\xi_{1}(t) \\
\xi_{2}(t)
\end{array}\right)
$$

donde $a \equiv\left(F-\gamma_{1}\right)>0$. Esta ecuación es consistente con la descripción teórica de la Sec. 4.2.2 si los parámetros: $\gamma_{2}=\omega \mathrm{y}\left|\mathrm{F}_{e}\right|=k_{e}\left|E_{e}\right|$. Para este caso se tiene que

$$
<h_{1}>=\frac{k_{e}\left|E_{e}\right|(a+D)}{(a+D)^{2}+\omega^{2}}, \quad<h_{2}>=\frac{k_{e}\left|E_{e}\right| \omega}{(a+D)^{2}+\omega^{2}},
$$

de tal forma que el parámetro $q^{2}$ es igual a

$$
q^{2}=\frac{\left(k_{e}\left|E_{e}\right|\right)^{2}}{(a+D)^{2}+\omega^{2}}
$$


siendo $\left|E_{e}\right|^{2}=E_{e_{1}}^{2}+E_{e_{2}}^{2}$. La varianza del número complejo $h$ es ahora

$$
\sigma^{2} \equiv<h h^{*}>-|<h>|^{2}=\sigma_{11}+\sigma_{22}=\frac{\epsilon}{a}+\frac{k_{e}^{2}\left|E_{e}\right|^{2} D}{a\left(a^{2}+\omega^{2}\right)}+\mathcal{O}\left(D^{2}\right) .
$$

El tiempo de paso asociado a (4.61) está dado por

$$
<t_{i}>=<t_{i}>_{0}+\frac{1}{2 a} \sum_{m=1}^{\infty} \frac{(-1)^{m} \beta^{2 m}}{m m !}
$$

o bien

$$
<t_{i}>=<t_{i}>_{0}-\left[E_{1}\left(\beta^{2}\right)-\psi(1)+\ln \left(\beta^{2}\right)\right]
$$

donde

$$
<t_{i}>_{0}=\frac{1}{2 a}\left\{\ln \left(\alpha^{2} R^{2}\right)-\psi(1)\right\}
$$

$\operatorname{con} \beta^{2}=\alpha^{2} q^{2}$.

Como podemos observar en la Fig. 4.7, existen dos regímenes para la distribución de los tiempos de paso. Cuando la constante de tiempo asociada a la difusión es más pequeña ( $D$ grande) que el tiempo de encendido, la teoría predice un comportamiento logarítmico con $\alpha^{2}$ y $\beta^{2}$ [9]. Cuando esta constante de tiempo es más grande ( $D$ pequeño) los efectos de difusión son despreciables. Además podemos observar, que existe un valor mínimo de los tiempos de encendido que divide estas dos regiones, el cual ocurre en un caso de resonancia, e.d. para un valor dado del parámetro de desintonización $\omega=\gamma_{2}$, el valor de la constante $D$ que hace mínimo el tiempo de encendido es tal que $D=\omega$. De modo que para un valor dado del parámetro $\omega$ entre el campo externo y el detector Láser, la resonancia puede ocurrir únicamente por fluctuaciones en la frecuencia del campo externo, tal que $D=\omega$.

Para concluir con esta sección, hagamos ahora las siguientes observaciones acerca del estudio de las dinámicas de los sistemas Láser (4.47) y (4.60) que otros autores han reportado en la literatura.

En el estudio de la detección de señales ópticas débiles de los sistemas Láser (4.47) y (4.60) de acuerdo con las Refs. [2, 9], se muestra que el formalismo estándar de TCD funciona apropiadamente para caracterizar tales sistemas, sin que los autores hayan podido explicar porqué el formalismo estándar de TCD funciona y es consistente en la descripción de un sistema que por naturaleza es rotacional. 


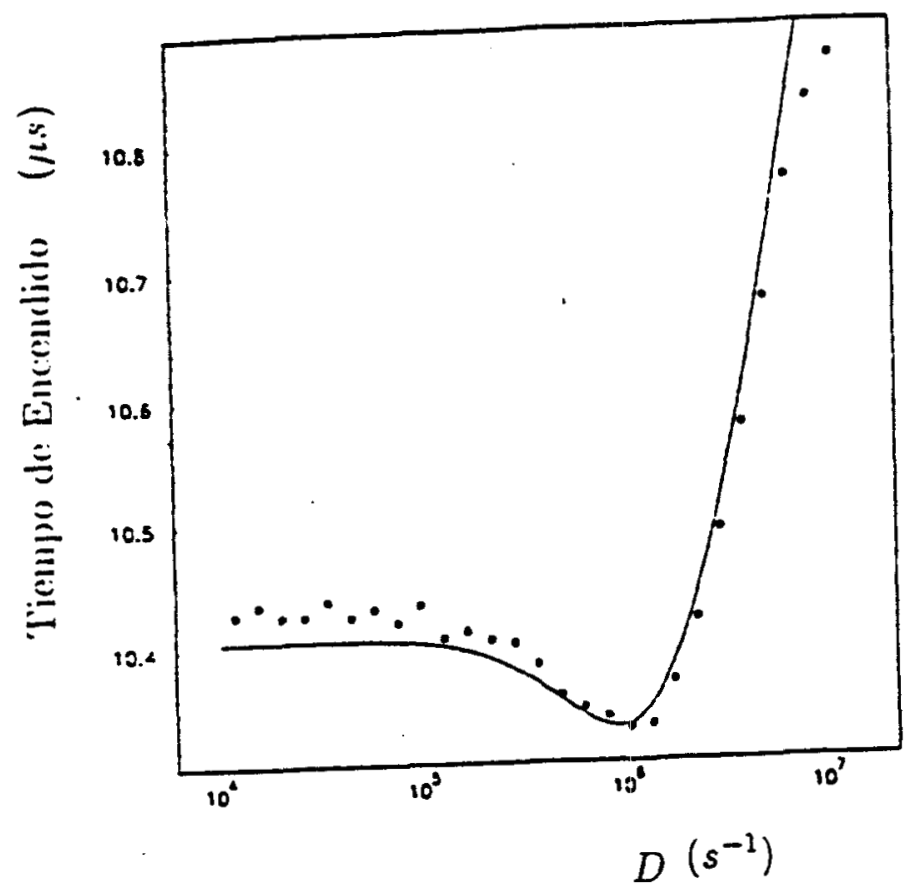

Fig. 4.7: Tiempo de encendido contra la constante de difusión $D$. La línea continua representa los resultados analíticos y los puntos corresponden a la simulación numérica de la Ref. [9].

Ahora ya sabemos con certeza el porqué la explicación anterior no puede darse en el esquema dinámico de (4.47) ó (4.60), pues éstos corresponden al esquema dinámico en el espacio $\mathbf{x}$ de nuestro trabajo de tesis, donde los efectos rotacionales de la fuerza sistemática siempre están presentes en el sistema. El mérito de nuestro trabajo consiste, entre otras cosas, en demostrar porqué el formalismo estándar de TCD funciona y además admite una formulación matricial generalizada que conduce prácticamente a los mismos resultados de la formulación estándar, en la escala de tiempos largos.

\subsection{Sistemas rotacionales de tres variables}

Para terminar este capítulo, estudiemos la caracterización dinámica de los sistemas de tres variables en presencia de fuerza externa constante, los cuales como verémos, muestran un comportamiento similar al caso de dos variables como se muestra en el apéndice A.3, es decir las matrices antisimétrica y de rotación están dadas por

$$
\mathbf{W}=\left(\begin{array}{ccc}
0 & \omega & 0 \\
-\omega & 0 & 0 \\
0 & 0 & 0
\end{array}\right), \quad \mathbf{R}(t)=\left(\begin{array}{ccc}
\cos \omega t & -\sin \omega t & 0 \\
\sin \omega t & \cos \omega t & 0 \\
0 & 0 & 1
\end{array}\right)
$$

donde $\omega^{2}=\omega_{1}^{2}+\omega_{2}^{2}+\omega_{3}^{2}$. La Fig. 4.8 muestra una trayectoria del sistema (4.68). 


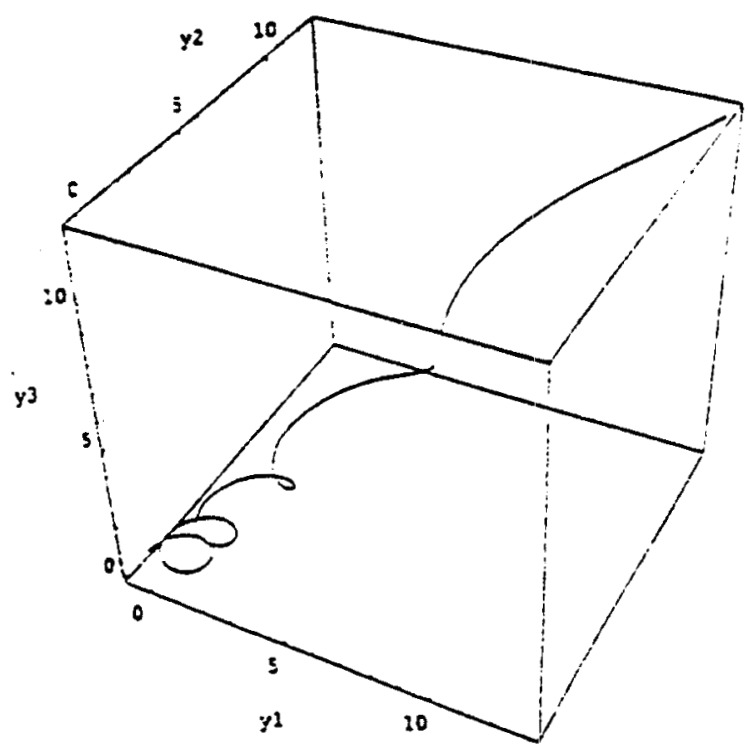

Fig. 4.8: Evolución dinámica de una trayectoria del sistema de tres variables en el espacio de coordenadas $\left(y_{1}, y_{2}, y_{3}\right)$.

Los valores medios de los elementos de la matriz constante $\mathbf{h}$ en este caso son

$$
<h_{1}>=\frac{f_{e_{1}} a}{a^{2}+\omega^{2}}-\frac{f_{e_{2}} \omega}{a^{2}+\omega^{2}}, \quad<h_{2}>=\frac{f_{e_{1}} \omega}{a^{2}+\omega^{2}}+\frac{f_{e_{2}} a}{a^{2}+\omega^{2}}, \quad<h_{3}>=\frac{f_{e_{3}}}{a} .
$$

Sin perder la generalidad podemos hacer que $f_{e_{3}}=0$, tal que el parámetro $q^{2}$ sea entonces

$$
q^{2}=\frac{\left|\mathbf{F}_{e}\right|^{2}}{a^{2}+\omega^{2}}
$$

el cual es similar a (4.27), pero ahora $\left|\mathbf{F}_{e}\right|^{2}=f_{e_{1}}^{2}+f_{e_{2}}^{2}+f_{e_{3}}^{2}$.

También se puede mostrar que la matriz $\sigma_{i j}$ es diagonal, es decir $\sigma_{i i}=\sigma^{2}$, tal que

$$
\sigma^{2}=\frac{Q}{a}
$$

Para este caso, la densidad de probabilidad marginal $P(h)$ se obtiene de (4.18), dando como resultado

$$
P(h)=\frac{2 \alpha}{\sqrt{\pi} q} h \sinh \left(2 \alpha^{2} q\right) \mathrm{e}^{-\alpha^{2}\left(h^{2}+q^{2}\right)}
$$

donde $\alpha^{2} \equiv \frac{1}{2 \sigma^{2}}$.

La caracterización lineal de tales sistemas a través de los tiempos de paso, es

$$
<t_{i}>=<t_{i}>_{0}+\frac{1}{2 a} \psi\left(\frac{3}{2}\right)-\frac{\mathrm{e}^{-\beta^{2}}}{2 a} \sum_{m=0}^{\infty} \frac{\beta^{2 m}}{m !} \psi\left(m+\frac{3}{2}\right)+\mathcal{O}(Q)+\mathcal{O}\left(q^{2}\right),
$$

Conde

$$
<t_{i}>_{0}=\frac{1}{2 a}\left\{\ln \left(\alpha^{2} R^{2}\right)-\psi\left(\frac{3}{2}\right)\right\}
$$




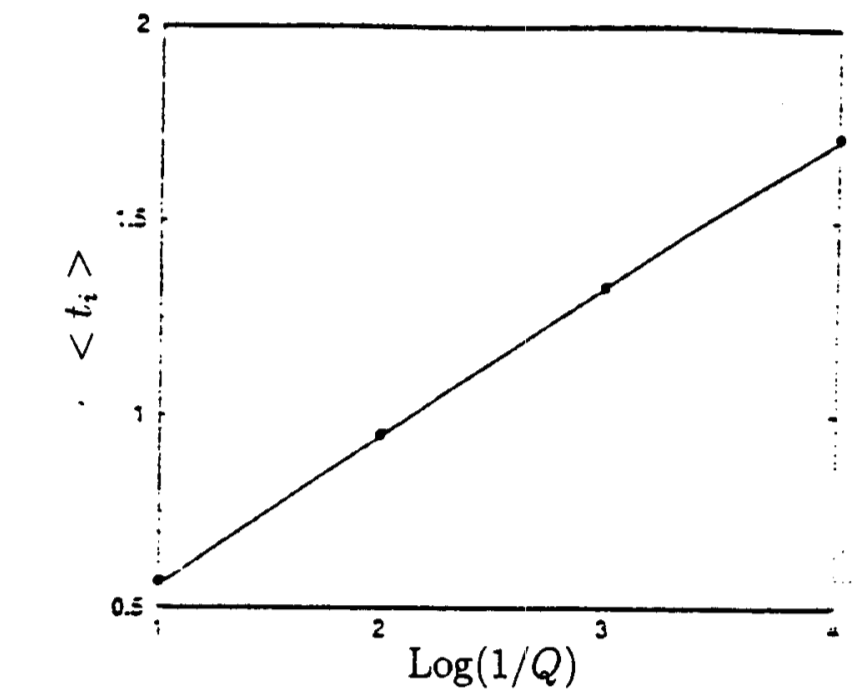

Fig. 4.9: Comparación entre la escala de tiempo (4.75) (línea continua) y la simulación numérica (puntos), para el caso $\left|F_{e}\right|=Q, a=3.0, \omega=6.0$ y $R=1.0$. Los resultados de simulación corresponden a $Q=10^{-1}, Q=10^{-2}, Q=10^{-3}, Q=10^{-4}$.

En el Apéndice C.2 se encuentra la demostración de la serie de (4.73) a una expresión más sencilla, que es fácil de mostrar su convergencia. Entonces la ecuación (4.73) puede escribirse de la siguiente forma

$$
<t_{i}>=<t_{i}>_{0}+\frac{\sqrt{\pi}}{2 a} \sum_{m=1}^{\infty} \frac{(-1)^{m} \beta^{2 m}}{2 m\left(m+\frac{1}{2}\right) !}+\mathcal{O}(Q)+\mathcal{O}\left(q^{2}\right),
$$

siendo $\left\langle t_{i}>_{0}\right.$ la misma expresión de (4.74). En la Fig. 4.9 mostramos la comparación de esta última expresión con los resultados obtenidos de la simulación numérica, mostrando una excelente concordancia.

El tiempo de relajación no lineal está dado por

$$
T_{N L}=T_{N L}^{0}+\frac{\sqrt{\pi}}{2 a} \sum_{m=1}^{\infty} \frac{(-1)^{m} \beta^{2 m}}{2 m\left(m+\frac{1}{2}\right) !},
$$

siendo $T_{N L}^{0}$ la escala de tiempo no lineal en ausencia de fuerza externa

$$
T_{N L}^{0}=<t_{i}>_{0}+I_{N L}
$$

donde $I_{N L}$ contiene las contribuciones no lineales del sistema.

De acuerdo con (4.76) y en analogía con el caso de dos variables se puede proponer un posible receptor de salida. 


\section{Capítulo 5}

\section{Conclusiones y perspectivas}

La teoría cuasi-determinista de la formulación estándar propuesta para caracterizar, en el límite de tiempos largos, los procesos de decaimiento de sistemas inestables de la forma (1.21) a través de los tiempos de paso y de los tiempos de ralajación no lineales ha sido generalizado para caracterizar a los sistemas inestables rotacionales de la forma (1.22) mediante un esquema matricial completamente general.

Podemos concluir que las escalas de tiempo para caracterizar la dinámica rotacional (3.1), a través de los momentos estadísticos $\left\langle r^{l}(t)\right\rangle$, son las mismas escalas de tiempo obtenidas para caracterizar la dinámica no rotacional (2.6). En la descripción de los sistemas rotacionales (4.1), los tiempos característicos son prácticamente iguales a los que caracterizan a la dinámica (2.65) de la formulación estándar. La razón principal del porqué estas escalas de tiempo son iguales o prácticamente iguales en la descripción mencionada anteriormente, es porque existen dinámicas equivalentes a (3.1) y (4.1) representadas por (3.61) y (4.2), respectivamente; las cuales se obtiene mediante una transformación lineal de coordenadas del espacio $\mathbf{x}$ al espacio de variables $\mathbf{y}$, de tal forma que en el espacio transformado de coordenadas $\mathbf{y}$, tales sistemas siguen siendo rotacionales. En el espacio transformado, la dinámica descrita por (3.61) es muy semejante a (2.6), en cambio la descrita por (4.2) es totalmente diferente a la de (2.65), sin embargo en ciertas escalas de tiempo de observación éstas dinámicas pueden ser consideras casi equivalentes, por las siguientes razones, que acontinuación discutiremos.

En ausencia de fuerza externa, la dinámica (3.61) es muy semejante a la dinámica (2.6), ya que la única diferencia entre ambas es la aparición de la fuerza fluctuante rotacional definida por $\mathbf{z}_{R}(t)=\mathbf{R}(t) \mathbf{z}(t)$ en (3.61), cuyo efecto es rotar las trayectorias del sistema 
alrededor del origen de coordenadas del espacio y de manera aleatoria. En particular esta situación ha sido mostrada con resultados de simulación numérica en las Figs. 3.3 y 3.4, para los sistemas rotacionales de dos variables únicamente en el régimen lineal de (2.6) y (3.61), respectivamente. Observamos claramente que las trayectorias estocásticas de ambas dinámicas son líneas rectas con la única diferencia de que en la dinámica lineal de (3.61), éstas líneas rectas están rotadas de manera aleatoria alrededor del origen de coordenadas del plano $\left(y_{1}, y_{2}\right)$, debido al efecto rotacional del ruido. Bajo éstas circunstancias podemos entender ahora porqué el promedio de los tiempos de paso son los mismos obtenidos en la descripción dinámica de (2.6). Una situación análoga ocurre en los sistemas tres variables de (2.6) y (3.61). En este caso las trayectorias estocásticas descritas por la parte lineal de dichos procesos, son también líneas rectas que emergen del origen de coordenadas en tres dimensiones.

En presencia de fuerza externa, la dinámica (4.2) es totalmente diferente a la de (2.65), ya que en este caso la fuerza externa $\mathbf{F}_{e}^{R}=\mathbf{R}(t) \mathbf{F}_{e}$ de (4.2) es también de carácter rotacional al igual que la fuerza fluctuante $\mathbf{z}_{\mathbf{R}}$. En esta situación las trayectorias estcásticas de proceso (4.2) no sólo están rotadas alrededor del origen de coordenadas de manera aleatoria, sino que también pueden generar trayectorias rotacionales en su evolución dinánica hasta alcanzar el valor de referencia preestablecido o el estado estacionario correspondiente, debido también al carácter rotacional de $\mathbf{F}_{e}$.

En el caso particular de los sistemas de dos variables, las trayectorias estocásticas correspondientes al proceso lineal de (4.2) ya no son las líneas rectas como las que se muestran en la Fig. 3.3, sino trayectorias estocásticas las cuales, aparte de rotar alrededor del origen de coordenadas aleatoriamente, emergen de dicho origen de coordenadas formando "bucles" en su evolución dinámica hasta alcanzar el valor de referencia $R=1.0$, debido precisamente a los efectos rotacionales de la fuerza externa; tal como se muestra en la Fig. 4.1. Esta descripción dinámica ha sido obtenida si la amplitud de la fuerza externa domina sobre la intesidad del ruido interno.

En el caso contrario, si la amplitud de la fuerza externa es igual a la intensidad del ruido interno y además consideramos la misma escala de tiempo de observación de la Fig. 4.1, podemos apreciar que los efectos rotacionales o "bucles" de dichas trayectorias son casi indistinguibles de tal forma que éstas aparentan ser vistas prácticamente como líneas rectas, como se muestra en la Fig. 4.2. En este caso la dinámica lineal de (4.2) es 
casi semejante a la dinámica lineal de (2.65) descrita también por un conjunto de líneas rectas. Naturalmente este efecto depende de la escala de tiempo de observación, ya que para tiempos no tan largos de observación los efectos rotacionales pueden ser importantes.

La descripción teórica de los argumentos arriba mencionados, ha sido sustentada en los Caps. 3 y 4 de la siguiente manera.

En el Cap. 3 hemos estudiado la formulación matricial de la teoría cuasi-determinista para caracterizar la dinámica de sistemas inestables rotacionales (3.1) inducidas por ruido blanco y ruido de color gaussianos. Para el caso de ruido blanco, se demuestra que el formalismo de TCD admite una descripción general para cualquier número de variables físicas y muestra también que las escalas de tiempo obtenidas en este esquema son las mismas, que las del Cap. 2 de la formulación estándar. En el caso de ruido de color, esto no es así, ya que la descripción de TCD requiere del número específico de variables para su descripción; debido al carácter no markoviano del problema que se manifiesta en las Ecs. (3.36) y (3.37). Se muestra para los sistemas de dos y tres variables que los requerimientos de TCD se satisfacen a primer orden de aproximación en el tiempo de correlación $\tau$ y en el límite de intensidad de ruido pequeño, de forma tal que los efectos del acoplamiento entre el parámetro de rotación $\omega$ y el tiempo de correlación $\tau$ pueden ser despreciados al inicio del proceso de decaimiento de dichos sistemas. Bajo estas condiciones las escalas de tiempo coinciden con las de la formulación estándar.

En el Cap.4, ha sido desarrrollado en el espacio transformado de coordenadas y, el formalismo generalizado de TCD para caracterizar el proceso de decaimiento de los sistemas (4.2) únicamente en el contexto de ruido blanco gaussiano.

En el caso de fuerza externa constante y para los sistemas rotacionales de dos y tres variables, se verifica que las escalas de tiempo son prácticamente las mismas que fueron obtenidas en la formulación estándar del Cap. 2; excepto por el parámetro $\beta^{2}=\alpha^{2} q^{2}$ que contiene el factor $q^{2}=\frac{\left|\mathbf{F}_{e}\right|^{2}}{a^{2}+\omega^{2}}$, el cual solamente renormaliza la amplitud de la fuerza externa. En el caso de tres variables $\omega^{2}=\omega_{1}^{2}+\omega_{2}^{2}+\omega_{3}^{2}$. Si $\omega=0$ obviamente las escalas de tiempo son exactamente las mismas.

Los resultados de la simulación numérica obtenida para los sistemas rotacionales de dos variables, sugiere analizar los límites de validez de las escalas de tiempo en las dos regiones de aproximación del parámetro $\beta^{2}$. Se demustra que si la amplitud de la fuerza externa domina sobre la intensidad del ruido interno $\beta^{2}>1$, entonces el formalismo 
generalizado de TCD no es apropiado para caracterizar la dinámica de tales sistemas, como se puede observar en la Fig. 4.1. Sin embargo, si la amplitud de la fuerza externa es débil tal que $\beta^{2} \leq 1$, entonces la descripción de TCD es consistente con la caracterización de dichos sistemas de acuerdo con la Fig. 4.2, lo cual era de esperarse. La consistencia del formalismo en el caso de tres variables se muestra en la Fig. 4.8

El formalismo desarrollado en el Cap. 4, ha sido aplicado al estudio de la dinámica transitoria de los sistemas Láser, particularmente en la Sec. 4.3.2, hemos utilizado los tiempos de relajación no lineales para calcular el receptor de salida del sistema Láser no lineal dada por la Ec. (4.47). Esta cantidad ha sido útil para detectar las señales ópticas débiles de otro Láser incidente de amplitud constante.

Por otro lado, si la fuerza externa es tal que fluctúa en el tiempo sólo a través de su fase, entonces de acuerdo con la Sec. 4.2.2, la descripción de TCD es apropiada si además de la aproximación de fuerza débil tal que $\beta^{2} \leq 1$, pedimos que la intensidad de las fluctuaciones de fase sean muy pequeñas. La teoría es compatible con el sistema Láser estudiado en la Sec. 4.3.3, cuyos resultados de los tiempos de paso coinciden con los de la Ref. [9].

Para finalizar nuestras conclusiones queremos hacer las siguientes observaciones que consideramos son necesarias e importantes para aclarar algunos de los conceptos no estudiados en las Refs. [61, 36, 2, 9], y por lo consiguiente nos motivó al desarrollo y restructuración de nuestro trabajo de tesis. La detección de las señales ópticas débiles del sistema Láser (4.47) ha sido ya estudiado en las Refs. [61, 36, 2] por otros autores, a través de los tiempos de paso y del formalismo estándar de TCD. Hemos mostrado que dicha ecuación es compatible con la Ec. (4.1) de nuestra formulación teórica. Sin embargo en ninguna de tales referencias antes mencionadas se hace mensión que el sistema Láser propuesto es un sistema rotacional y menos aún del porqué la formulación estándar de TCD funciona en la descripción dinámica de dicho sistema. Tales cuestionamientos han sido aclarados en el desarrollo de esta tesis.

Por otro lado, en $1993 \mathrm{~J}$. Dellunde et. al. [8] propusieron un método alternativo para detectar señales ópticas cuyas magnitudes son mayores que la intensidad del ruido interno a través de los tiempos de paso, tomando en cuenta los efectos rotacionales del sistema Láser (4.47). El punto importante que queremos resaltar de dicho trabajo es que el marco teórico propuesto no aclara con detalle lo siguiente. En dicho artículo se propone la Ec. 
(4.47) la cual es compatible con la Ec. (4.1) en el caso de dos variables $\left(x_{1}, x_{2}\right)$. En este espacio las trayectorias estocásticas de la dinámica lineal de (4.47) son trayectorias espirales que emergen del origen de coordenadas del plano complejo $\left(E_{1}, E_{2}\right)$, véase la Fig. 3.1; pero no corresponden a la trayectorias rotacionales en forma de "bucles" como se muestra en dicha referencia y se muestra en la Fig. 4.1. ¿De dónde y cómo surge esta trayectoria dinámica? no fue explicado en ninguna parte de dicho trabajo por los autores, aunque la descripción teórica haya sido correcta. Ahora sabemos porqué dicha descripción es correcta pues la dinámica de Langevin en el espacio $\mathbf{x}$ es totalmente equivalente a la dinámica de Langevin en el espacio de variables y. Esta nueva descripción dinámica que es muy necesaria para poder explicar los puntos antes mencionados, no había sido tomada en cuenta en ninguna de las referencias arriba citadas.

Queremos concluir con las perspectivas de nuetro trabajo con lo siguiente: Recientemente la caracterización dinámica de los sistemas inestables rotacionales (1.22) a través de la distribución de los tiempos de paso, en la región de fuerza externa débil $\beta^{2} \leq 1$, ha sido estudiado en la Ref. [30] para los sistemas de dos y tres variables; mientras en la región de fuerza externa fuerte $\beta^{2}>1$, la caracterización dinámica ha sido estudiado en la Ref. [31] sólo para los sistemas de dos variables. Dichos estudios, aunque hayan sido realizados para los sistemas de dos y tres variables, no fueron desarrollados en el esquema actual de esta tesis, es decir, en el marco teórico de las propiedades generales de las matrices de rotación. Por lo que nuestro propósito a corto plazo será estudiar la caracterización de la dinámica de Langevin rotacional en el contexto teórico generalizado del presente tarabajo, de manera que los sistemas rotacionales de dos y tres variables serán sólo casos particulares.

La descripción teórica en el caso de tres variables puede aplicarse posiblemente al estudio de la dinámica de partículas cargadas inmersas en un campo magnético externo, ya que en ciertas circunstancias, la trayectoria dinámica de una partícula cargada puede describir un comportamiento similar a la trayectoria dinámica que se muestra en la Fig. 3.2. En este sentido el estudio de la dinámica de plasmas puede ser un tema de interés.

Por otro lado, la dinámica de Langevin rotacional admite también una formulación covariante del campo electromagnético, por lo que ambos esquemas, la física de plasmas y la formulación covariante, serán nuestras perspectivas a futuro. 


\section{APENDICE A}

\section{Propiedades de la matriz antisimétrica $\mathrm{W}$}

\section{A.1 Demostración de $\mathrm{e}^{\mathrm{W} t}=\mathbf{R}(t)$}

Demostremos ahora que $\mathrm{e}^{\mathbf{W} t}$ es una matriz de rotación, que definimos como $\mathbf{R}(t)$. La demostración que presentamos para $\mathbf{R}(t)$ no hubiera sido posible sin la ayuda de los resultados reportados en las Refs. [47, 48].

Para tal propósito partimos de la siguiente expresión

$$
\mathbf{R}(t)=\mathrm{e}^{\mathbf{W} t}=\mathrm{e}^{(\Phi \mathbf{n} \times) t}=\mathbf{E}+\sum_{j=1}^{\infty} \frac{[(\Phi \mathbf{n} \times) t]^{j}}{j !}
$$

donde $\Phi$ y $\mathbf{n}$ son el ángulo y el eje de rotación, respectivamente; $\mathbf{E}$ es la matriz unidad. El desarrollo de la serie de potencia se puede separar en potencias pares e impares de la matriz que son multiplos de dos matrices, tal que la ecuación anterior se puede escribir como

$$
\mathbf{R}(t)=\mathbf{E}+\sum_{j=1}^{\infty} \frac{[(\Phi \mathbf{n} \times) t]^{2 j}}{(2 j) !}+\sum_{j=0}^{\infty} \frac{[(\Phi \mathbf{n} \times) t]^{2 j+1}}{(2 j+1) !}
$$

Haciendo el algebra pertinente se demuestra que

$$
\mathbf{R}(t)=\mathbf{E}+\left(\sum_{j=1}^{\infty} \frac{(-1)^{j}[\Phi t]^{2 j}}{(2 j) !}\right)\left[\mathbf{E}-\mathbf{n} \mathbf{n}^{T}\right]+\left(\sum_{j=0}^{\infty} \frac{(-1)^{j}[\Phi t]^{2 j+1}}{(2 j+1) !}\right) \mathbf{n} \times
$$

o bien

$$
\mathbf{R}(t)=\mathbf{E}+[\cos (\Phi t)-1]\left[\mathbf{E}-\mathbf{n} \mathbf{n}^{T}\right]+\sin (\Phi t) \mathbf{n} \times
$$

Por lo tanto, la matriz de rotación $\mathbf{R}(t)$ es igual a 


$$
\mathbf{R}(t)=\mathbf{n n}^{T}+\cos (\Phi t)\left[\mathbf{E}-\mathbf{n n}^{T}\right]+\sin (\Phi t) \mathbf{n} \times,
$$

la cual se conoce como la forma de Gibbs de la matriz de rotación.

Retomando la expresión (A.4) para escribirla de la siguiente manera

$$
\mathbf{R}(t)=\mathbf{E}+\sum_{j=1}^{\frac{N-C}{2}}\left\{\left[\cos \left(\Phi_{j} t\right)-1\right] S_{j}+\sin \left(\Phi_{j} t\right) A_{j}\right\}
$$

donde $S_{j}=\left[\mathbf{E}-\mathbf{n} \mathbf{n}^{T}\right]$ es un proyector y $A_{j}=\mathbf{n} \times$ es real y antisimétrica. Como podemos observar la sumatoria va desde $j=1$ hasta $\frac{N-C}{2}$ siendo $C$ el número de eigenvectores reales linealmente independientes que corresponden al eigenvalor cero.

La matriz $\mathbf{R}(t)$ tiene la propiedad de ser una matriz ortogonal, es decir

$$
\mathbf{R}(t) \mathbf{R}^{T}(t)=\mathbf{E},
$$

o términos de sus componentes

$$
\sum_{i} R_{j i}(t) R_{k i}(t)=\delta_{j k}
$$

La expresión (A.6) se puede factorizar en el producto abeliano

$$
\mathbf{R}(t)=\prod_{j=1}^{\frac{N-C}{2}}\left\{\mathbf{E}+\left[\cos \left(\Phi_{j} t\right)-1\right] S_{j}+\sin \left(\Phi_{j} t\right) A_{j}\right\} .
$$

Los vectores propios de $\mathbf{W}$ son también vectores propios de $\mathbf{R}(t)$, con valores propios correspondientes $\mathrm{e}^{i \Phi_{j} t}$.

El producto de $\mathbf{R}(t)$ con las parte real e imaginaria de un vector propio nos da

$$
\begin{aligned}
& \mathbf{R}(t) R_{j}=\cos \left(\Phi_{j} t\right) R_{j}-\sin \left(\Phi_{j} t\right) I_{j} \\
& \mathbf{R}(t) I_{j}=\sin \left(\Phi_{j} t\right) R_{j}+\cos \left(\Phi_{j} t\right) I_{j},
\end{aligned}
$$

o bien

$$
\mathbf{R}(t)\left(\begin{array}{c}
R_{j} \\
I_{j}
\end{array}\right)=\left(\begin{array}{cc}
\cos \left(\Phi_{j} t\right) & -\sin \left(\Phi_{j} t\right) \\
\sin \left(\Phi_{j} t\right) & \cos \left(\Phi_{j} t\right)
\end{array}\right)\left(\begin{array}{c}
R_{j} \\
I_{j}
\end{array}\right)
$$

Por lo tanto la matriz de rotación $\mathbf{R}(t)$ nos representa un conjunto de $\frac{N-C}{2}$ rotacioncs de ángulos $\Phi_{j}$ en los planos determinados por los dos vectores $R_{j}$ e $I_{j}$. 


\section{A.2 Demostración de $\mathbf{W} \mathbf{x}=\vec{w} \times \mathbf{x}$}

En este apéndice vamos a mostrar que el producto $\mathbf{W x}$, para el caso de tres dimensiones, siempre puede escribirse como el producto vectorial de dos vectores; dicho en otras palabras, toda matriz antisimétrica en el espacio de tres dimensiones determina un vector $\vec{w}$ tal que el producto matricial de la matriz antisimétrica es igual al producto vectorial del vector $\vec{w}$, es decir $\mathbf{W} \mathbf{x}=\vec{w} \times \mathbf{x}$, donde $\times$ representa el producto vectorial y $\vec{w}$ es el vector velocidad angular [48]. Si W es una matriz antisimétrica, entonces

$$
\mathbf{W}=\vec{w} \times
$$

donde

$$
\mathbf{W}=\left(\begin{array}{ccc}
0 & -w_{3} & w_{2} \\
w_{3} & 0 & -w_{1} \\
-w_{2} & w_{1} & 0
\end{array}\right), \quad \vec{w}=\left(\begin{array}{l}
w_{1} \\
w_{2} \\
w_{3}
\end{array}\right) .
$$

Además se puede verificar que para cualquier vector $\mathbf{x}$

$$
\mathbf{W} \mathbf{x}=\vec{w} \times \mathbf{x}
$$

lo cual es muy fácil de verificar, como se puede ver

$$
\mathbf{W} \mathbf{x}=\left(\begin{array}{ccc}
0 & -w_{3} & w_{2} \\
w_{3} & 0 & -w_{1} \\
-w_{2} & w_{1} & 0
\end{array}\right)\left(\begin{array}{l}
x_{1} \\
x_{2} \\
x_{3}
\end{array}\right)=\left(\begin{array}{c}
w_{2} x_{3}-w_{3} x_{2} \\
w_{3} x_{1}-w_{1} x_{3} \\
w_{1} x_{2}-w_{2} x_{1}
\end{array}\right)=\vec{\omega} \times \mathbf{x}
$$

\section{A.3 La matriz $W$ para el caso de tres variables}

En este apartado vamos a mostrar cómo la matriz antisimétrica $\mathbf{W}$, para el caso de tres variables, es muy similar a la matriz antisimétrica de dos variables.

Explicítamente la matriz $\mathbf{W}$ para el caso de tres variables, puede escribirse como

$$
\mathbf{W}=\left(\begin{array}{ccc}
0 & -\omega_{3} & \omega_{2} \\
\omega_{3} & 0 & -\omega_{1} \\
-\omega_{2} & \omega_{1} & 0
\end{array}\right)
$$

La similitud con el caso de dos variables se puede lograr mediante de una transformación de coordenadas, a través de una matriz de rotación $\mathbf{T}$; la cual se obtiene de la siguiente forma: los vectores columna asociados con la matriz $\mathrm{T}$ serán dados por los eigenvectores 
asociados con la matriz $\mathbf{W}$ [48]. Los eigenvalores de la matriz $\mathbf{W}$ son: $\lambda_{1}=0, \lambda_{2}=i \omega \mathrm{y}$ $\lambda_{3}=-i \omega$ donde $\omega$ se define como $\omega^{2} \equiv \omega_{1}^{2}+\omega_{2}^{2}+\omega_{3}^{2}$. Sus correspondientes eigenvectores son respectivamente

$$
\mathbf{v}_{\mathbf{1}}=\left(\begin{array}{c}
\omega_{1} \\
\omega_{2} \\
\omega_{3}
\end{array}\right), \quad \mathbf{v}_{\mathbf{2}}=\left(\begin{array}{c}
-\omega_{1} \omega_{3}-i \omega \omega_{2} \\
-\omega_{2} \omega_{3}+i \omega \omega_{1} \\
\omega_{1}^{2}+\omega_{2}^{2}
\end{array}\right), \quad \mathbf{v}_{\mathbf{3}}=\mathbf{v}_{\mathbf{2}}{ }^{*}
$$

donde el astérisco denota el complejo conjugado. Como podemos ver un eigenvector es real, como consecuencia del eigenvalor $\lambda_{1}=0$; los otros dos son el complejo conjugado uno del otro, debido que a sus eigenvalores que también son el complejo conjugado uno del otro.

Por lo tanto la matriz de rotación $\mathbf{T}$ es igual a

$$
\mathbf{T}=\frac{1}{\sqrt{\omega_{1}^{2}+\omega_{2}^{2}}}\left(\begin{array}{ccc}
\frac{-\omega_{1} \omega_{3}}{\omega} & -\omega_{2} & \frac{\omega_{1} \sqrt{\omega_{1}^{2}+\omega_{2}^{2}}}{\omega} \\
\frac{-\omega_{2} \omega_{3}}{\omega} & \omega_{1} & \frac{\omega_{2} \sqrt{\omega_{1}^{2}+\omega_{2}^{2}}}{\omega} \\
\frac{\omega_{1}^{2}+\omega_{2}^{2}}{\omega} & 0 & \frac{\omega_{3} \sqrt{\omega_{1}^{2}+\omega_{2}^{2}}}{\omega}
\end{array}\right),
$$

donde la primera y segunda columna corresponden a la parte real e imaginaria respectivamente del vector unitario $\hat{\mathbf{v}}_{2}$, y la tercera columna se debe al vector unitario $\hat{\mathbf{v}}_{1}$. Bajo la siguiente transformación $\tilde{\mathbf{T}} \mathbf{W}$ T se puede muestrar que

$$
\mathbf{W}^{\prime}=\tilde{\mathbf{T}} \mathbf{W} \mathbf{T}=\left(\begin{array}{ccc}
0 & \omega & 0 \\
-\omega & 0 & 0 \\
0 & 0 & 0
\end{array}\right)
$$

la cual es también una matriz antisimétrica similar al caso de dos variables. Con este resultado se simplifica el problema de tres variables al caso de dos variables. 


\section{APENDICE B}

\section{Fundamentos de la dinámica rotacional de Langevin}

\section{B.1 Relaciones de Reciprocidad de Onsager-Casimir}

La ecuación de Langevin rotacional propuesta en (3.1) tiene su origen en las Ecuaciones de Moviento Microscóspico (EMM) que son invariantes ante inversión y traslación temporal, como lo demostraron Onsager y Casimir.

Onsager [41, 42] mostró que la invariancia ante una inversión temporal de las ecuaciones microscópicas de movimiento (clásica o cuántica) implica que la matriz $\mathbf{L}$ de los coeficientes de transporte no es arbitraria, sino que es una matriz simétrica. Posteriormente Casimir [6] generalizó los resultados de Onsager, de ahí el nombre de Relaciones de Reciprocidad de Onsager-Casimir (RRO-C) [13]. Los resultados que presentamos en este apéndice son una generalización de los de Onsager-Casimir y son tomados de la tesis Doctoral del Dr. J.L. del Río Correa [7].

La demostración de las (RRO-C) está basada en las siguientes tres hipótesis a saber

I) La hipótesis de regresión de fluctuaciones, la cual afirma que en un sistema añejado ${ }^{1}$, las fluctuaciones se comportan en promedio siguiendo las leyes fenomelógicas.

II) Las relaciones constitutivas son lineales entre flujos y fuerzas termodinámicas.

III) La función de distribución de las fluctuaciones alrededor del estado de equilibrio es una gaussiana.

Puesto que las RRO-C se obtienen en una escala de tiempo mesoscópica, entonces

\footnotetext{
${ }^{1}$ Un sistema añejado es un sistema aislado que se ha dejado que relaje al estado de equilibrio termostático y los tiempos en los cuales se estará observando a este sistema son muy grandes comparados con sus tiempos de relajación natural.
} 
primero vamos a definir dicha escala, tal que

$$
\tau_{m}<<t<T_{M}
$$

donde $\tau_{m}$ representa el tiempo microcópico, como el tiempo de colisión; y $T_{M}$ es el tiempo macroscópico, como el tiempo de relajación.

Con la ayuda de las hipótesis I) y II) Onsager demostró que las variables de regresión ${ }^{2}$ en una escala mesoscópica de tiempo son dadas por

$$
\mathbf{a}(t ; \mathbf{b})=\mathbf{b}+t \mathbf{L} \mathbf{\Phi}(\mathbf{b})
$$

donde $\mathbf{b}$ representa al estado inicial de no equilibrio al tiempo $t=0$, $\mathbf{L}$ la matriz de coeficientes de transporte y $\boldsymbol{\Phi ( b )}$ es la fuerza de Onsager.

La tercera hipótesis consiste en

$$
g_{e q}(\mathbf{b})=A \mathrm{e}^{\left[-\frac{1}{2} \mathbf{c}^{-1}(0): \mathbf{b b}\right]},
$$

siendo $A$ una constante de normalización y $\mathbf{c}^{-1}(0)$ denota la matriz de correlación al tiempo cero. Por lo que el vector $\boldsymbol{\Phi ( b )}$ está dado por

$$
\Phi(\mathbf{b})=\mathbf{c}^{-1}(0) \mathbf{b} \text {. }
$$

Por lo tanto, la expresión (B.2) es igual a

$$
\mathbf{a}(t ; \mathbf{b})=\mathbf{b}+t \mathbf{M} \mathbf{b}
$$

donde $\mathbf{M} \equiv \mathbf{L} \mathbf{c}^{-1}(0)$. Multiplicando esta expresión por $\mathbf{b}$ y tomando el promedio con la función de distribución de equilibrio, obtenemos que la función de correalción es

$$
\mathbf{c}(t)=\langle\mathbf{a}(t ; \mathbf{b}) \mathbf{b}>=<\mathbf{b} \mathbf{b}>-t \mathbf{M}<\mathbf{b} \mathbf{b}>\text {. }
$$

Haciendo uso de la expresión (B.3) se demuestra que $<\mathbf{b b}>=\mathbf{c}(0)$, entonces la ecuación anterior nos queda de la siguiente manera

$$
\mathbf{c}(t)=\mathbf{c}(0)-t \mathbf{M} \mathbf{c}(0) .
$$

\footnotetext{
${ }^{2} \mathrm{~A}$ estas variables en la literatura se les conoce como Variables de Regresión de Onsager (VRO), las cuales describen en promedio la regresión del sistema al estado de equilibrio cuando el sistema parte de un estado inicial de no-equilibrio, al cual ha llegado espontáneamente por medio de una fluctuación nat ural.
} 
Esta expresión se ha obtenido haciendo uso de las hipótesis de Onsager, por lo que es una expresión semi-fenomenológica. Este resultado debe ser compatible con las ecuaciones microscópicas de movimiento, de manera que la matriz de correlación (B.7) debe satisfacer las relaciones de simetría y antisimetría, que son una consecuencia de las invariancias de las ecuaciones de movimiento ante inversión y traslación temporal.

Como consecuencia de la invariancia de las ecuaciones de movimiento ante inversión temporal, implica que la matriz de correlación satisface

$$
\mathbf{c}(t)=\Lambda \mathbf{c}^{T}(t) \Lambda
$$

donde la matriz $\Lambda \equiv \lambda_{i} \delta_{i, j}$ y $\lambda_{i}= \pm 1$ según la paridad de $\mathbf{a}(t ; \mathbf{b})$.

En tanto que la invariancia de las EMM ante un corrimiento en el origen del tiempo, la matriz de correlación satisface que

$$
\mathbf{c}(t)=\mathbf{c}^{T}(-t)
$$

El pedir que la matriz de correlación (B.7) satisfaga la relación de simetría (B.8) implica obtener la simetría de la matriz de coeficientes de transporte $\mathbf{L}$, conocida en la literatura como las Relaciones de Reciprocidad de Onsager-Casimir.

Sustituyendo la Ec. (B.7) en la Ec. (B.8) se obtiene

$$
[\mathbf{U}-t \mathbf{M}] \mathbf{c}(0)=\Lambda\left[\mathbf{c}^{T}(0)\left(\mathbf{U}-t \mathbf{M}^{T}\right)\right] \Lambda
$$

De la Ec. (B.8) para $t=0$ se tiene que

$$
\mathbf{c}(0)=\Lambda \mathbf{c}^{T}(0) \Lambda
$$

con ayuda de esta ecuación la expresión (B.10) se simplifica, tal que

$$
\mathbf{M} \mathbf{c}(0)=\Lambda \mathbf{c}^{T}(0) \mathbf{M}^{T} \Lambda
$$

Recordando que $\mathbf{M} \equiv \mathbf{L} \mathbf{c}^{-1}(0)$, obtenemos que la matriz de coeficientes de transporte posee la siguiente

$$
\mathbf{L}=\Lambda \mathbf{L}^{T} \Lambda .
$$

Esta ecuación representa las llamadas Relaciones de Reciprocidad de OnsagerCasimir. 
De manera que el teorema de Onsager asegura que la invariancia de las ecuaciones microscopicas del movimiento ante inversión temporal, se refleja Macroscopicamente en la simetría de la matriz de coeficientes de transporte, dada por la ecuación (B.13).

Veamos que pasa con la simetría de la matriz de los coeficientes de transporte cuando la ecuación (B.7) satisface la propiedad (B.9), es decir

$$
\mathbf{c}(0)-t \mathbf{M} \mathbf{c}(0)=\mathbf{c}^{T}(0)+t \mathbf{c}^{T}(0) \mathbf{M}^{T} \text {. }
$$

De la misma Ec. (B.9) podemos ver que para $t=0$ la matriz $\mathbf{c}(0)$ es simétrica, entonces la ecuación anterior nos queda de la siguiente forma

$$
-\mathbf{M} \mathbf{c}(0)=\mathbf{c}^{T}(0) \mathbf{M}^{T},
$$

y utilizando nuevamente la definición de la matriz $\mathbf{M} \equiv \mathbf{L} \mathbf{c}^{-1}(0)$, se demuestra que

$$
\mathbf{L}=-\mathbf{L}^{T}
$$

donde $\mathbf{L}$ es una matriz antisimétrica que vamos llamarle $\mathbf{W}$ para no confundirla con la matriz simétrica de Onsager.

$$
\mathbf{W}=-\mathbf{W}^{T}
$$

Por lo tanto podemos concluir que cuando sólo se toma en cuenta la invariancia de las EMM ante una traslación en el tiempo, ésto se ve reflejado macroscópicamente en la matriz de los coeficientes de transporte $\mathbf{W}$ que representa una matriz antisimétrica. 


\section{APENDICE C}

\section{Cálculo Analítico de las series (2.84) y (2.92)}

\section{C.1 Dos variables}

La serie de (2.82) dada por

$$
e^{-\beta^{2}} \sum_{m=0}^{\infty} \frac{\beta^{2 m}}{m !} \psi(m+1)
$$

puede reducirse a la expresión (2.84). Para ello partimos de la función Hipergeométrica degenerada definida como

$$
M(\alpha, \gamma ; z)=\sum_{m=0}^{\infty} \frac{\alpha_{m} z^{m}}{\gamma_{m} m !}
$$

donde $\alpha_{m}=\frac{\Gamma(m+\alpha)}{\Gamma(\alpha)}$ y $\gamma_{m}=\frac{\Gamma(m+\gamma)}{\Gamma(\gamma)}$ [14]. Si hacemos que: $\alpha=\lambda+1, \gamma=1$ y $z=\beta^{2}$; y usamos la definición $\Gamma^{\prime}(x)=\Gamma(x) \psi(x)$, podemos mostrar que

$$
\left.\frac{d}{d \lambda} M\left(\lambda+1,1 ; \beta^{2}\right)\right|_{\lambda=0}=\sum_{m=0}^{\infty} \frac{\beta^{2 m}}{m !}[\psi(m+1)-\psi(1)] .
$$

Por otro lado, si usamos la siguiente identidad

$$
M(\alpha, \gamma ; z)=e^{z} M(\gamma-\alpha, \gamma ;-z),
$$

podemos también verificar que se satisface la siguiente propiedad

$$
\left.\frac{d}{d \lambda} M\left(\lambda+1,1 ; \beta^{2}\right)\right|_{\lambda=0}=\left.e^{\beta^{2}} \frac{d}{d \lambda} M\left(-\lambda, 1 ;-\beta^{2}\right)\right|_{\lambda=0} .
$$

Con la identidad (C.2), se puede mostrar lo siguiente

$$
M\left(-\lambda, 1 ;-\beta^{2}\right)=1-\lambda\left[\sum_{m=1}^{\infty} \frac{(-1)^{m} \beta^{2 m}}{m m !}\right]+\mathcal{O}\left(\lambda^{2}\right),
$$


y por tanto

$$
\left.\frac{d}{d \lambda} M\left(-\lambda, 1 ;-\beta^{2}\right)\right|_{\lambda=0}=-\sum_{m=1}^{\infty} \frac{(-1)^{m} \beta^{2 m}}{m m !} .
$$

De acuerdo a (C.3), (C.5) y (C.7) podemos concluir que

$$
e^{-\beta^{2}} \sum_{m=0}^{\infty} \frac{\beta^{2 m}}{m !} \psi(m+1)=\psi(1)-\sum_{m=1}^{\infty} \frac{(-1)^{m} \beta^{2 m}}{m m !}
$$

que corresponde a la serie de (2.84). En este caso particular se puede mostrar que (C.8) es igual a

$$
\sum_{m=1}^{\infty} \frac{(-1)^{m}}{m m !} x^{m}=-\left[E_{1}(x)-\psi(1)+\ln (x)\right] .
$$

donde $E_{1}(x)$ es la función exponencial integral [1].

\section{C.2 Tres variables}

Finalmente para el caso de tres variables, se puede demostrar que la serie de (2.90) se reduce a la de (2.92) siguiendo los mismos pasos algebraicos dados anteriormente. Para lograr esto únicamente debemos cambiar los valores de los parámetros $\alpha$ y $\gamma$, ya que $z$ sigue siendo la misma expresión $z=\beta^{2}$, entonces si $\alpha=\lambda+\frac{3}{2}$ y $\gamma=\frac{3}{2}$, se llega a

$$
e^{-\alpha^{2} q^{2}} \sum_{m=0}^{\infty} \frac{\left(\alpha^{2} q^{2}\right)^{m}}{m !} \psi\left(m+\frac{3}{2}\right)=\psi\left(\frac{3}{2}\right)-\sqrt{\pi} \sum_{m=1}^{\infty} \frac{(-1)^{m}\left(\alpha^{2} q^{2}\right)^{m}}{2 m\left(m+\frac{1}{2}\right) !}
$$

que corresponde a la serie de (2.92). 


\section{Referencias}

[1] M. Abramowitz and I. A. Stegun, Handbook of Mathematical Functions (Dover, New York 1972).

[2] S. Balle, F. de Pascuale and M. San Miguel, Phys. Rev. A41, 5012 (1990).

[3] R. W. Boyd, M.G. Raymer and L.M. Nardicci, Optical Instabilities (Cambridge Press 1986).

[4] J. Casademunt, J. I. Jiménez-Aquino and J. M:^Sancho, Physica A156, 628 (1989).

[5] J. Casademunt, J. I. Jiménez-Aquino and J. M. Sancho, Phys. Rev. A40, 5905 (1989).

[6] H. B. G. Casimir, Rev. Mod. Phys. 17, 343 (1945).

[7] J. L. del Río Correa, Principios Básicos de la Mecánica Estadística de Procesos Irreversibles, Tesis Doctoral (UAM-Izt., México D.F. 1989).

[8] J. Dellunde, M. C. Torrent and J. M. Sancho, Opt. Comm. 102, 277 (1993).

[9] J. Dellunde, M. C. Torrent, J. M. Sancho and M. San Miguel, Opt. Comm. 109, 435 (1994).

[10] S. N. Dixit and P. S. Sahni, Phys. Rev. Lett. 50, 1273 (1983).

[11] A. Einstein, Ann. d. Physik 17, 549 (1905).

[12] R. F. Fox, Physics Reports (Review Section of Physics Letters) 48. No. 48, 179 (1978).

[13] S. R. de Grood and P. Mazur, Non-Equilibrium Thermodynamics (Dover, New York 1984). 
[14] I. S. Gradshteyn and I. M. Ryzhik, Table of integrals series and products (Academic Press, New York 1965).

[15] C. W. Gardiner, Handbook of stochastic method (Springer, Berlin 1983).

[16] J. D. Gunton, M. San Miguel and P. sahni, Phase Transitions and Critical Phenomena, Vol. 8 ( Eds. C. Domb and J. Lebowitz, Academic Press, 1983)

[17] F. Haake, J. W. Haus and R. Glauber, Phys. Rev. A23, 3255 (1981).

[18] W. Horsthemke and R. Lefever, Noise Induced Transitions (Springer Verlag, 1983).

[19] J. I. Jiménez-Aquino, J. Casademunt and J. M. Sancho, Phys. Lett. A133, 364 (1988).

[20] J. I. Jiménez-Aquino and J. M. Sancho, Phys. Rev. A43, 589 (1991).

[21] J. I. Jiménez-Aquino and J. M. Sancho, J. Phys. A: Math. Gen. 25, 6179 (1992).

[22] J. I. Jiménez-aquino, J. M. Sancho and J. Casademunt, Physica A195, 163 (1993).

[23] J. I. Jiménez-Aquino and J. M. Sancho, Phys. Rev. E47, 1558 (1993).

[24] J. I. Jiménez-Aquino, J. Phys. A27, 4745 (1994).

[25] J. I. Jiménez-Aquino, Opt. Comm. 114, 101 (1995).

[26] J. I. Jiménez-Aquino, Physica A229 (1996).

[27] J. I. Jiménez-Aquino, Emilio cortés and P. Orea, Physica A232, 229 (1996).

[28] J. I. Jiménez-Aquino, Physica A237, 113 (1997).

[29] J. I. Jiménez-Aquino, Physica A245, 503 (1997).

[30] J. I. Jiménez-Aquino and M. Romero-Bastida, Physica A292, 153 (2001).

[31] J. I. Jiménez-Aquino, Emilio Cortes and N. Aquino, Physica A (en imprenta) (2001).

[32] P. Jung, G. Vemuri and R. Roy, Optics Comm. 78, 58 (1990).

[33] K. Kaminishi, R. Roy, R. Short and L. Nandel, Phys. Rev. A 24, 370 (1981). 
[34] S. Koch, Dynamics of First-Orden Phase Transitions (Lecture Notes in Physics, 183. Springer Verlag 1983).

[35] P. Langevin, Comptes. Rendues. Acad. Sci. Paris 146, 530 (1908).

[36] I. Littler, S. Balle, K. Bergmann, G. Vemuri and R. Roy, Phys. Rev. A41, 4131 (1990).

[37] P. Orea and J. I. Jiménez-Aquino, Rev. Mex. de Fís. 44(3), 245 (1998).

[38] P. Orea and J. I. Jiménez-Aquino, Physica A258, 89 (1998).

[39] P. Orea and J. I. Jiménez-Aquino, Physica A258, 101 (1998).

[40] P. Orea and J. I. Jiménez-Aquino, Physica A273, 315 (1999).

[41] L. Onsager, Phys. Rev. 37, 405 (1931); 38, 2265 (1931).

[42] L. Onsager and S. Machlup, Phys. Rev. 91, 1505 (1953).

[43] F. de Pascuale and P. Tombesi, Phys. Lett. A72, 7 (1979).

[44] F. de Pascuale, J. M. Sancho, M. San Miguel and P. Tartaglia, Phys. Rev. A33, 4360 (1980).

[45] F. de Pascuale, J. M. Sancho, M. San Miguel and P. Tartaglia, Phys. Rev. Lett. 56, $2473(1986)$.

[46] J. Perrin, Atoms (Constable, London, 1916).

[47] E. Piña, Acta Mexicana de Ciencia y Tecnología, IPN, México, Vol. VII, 55 (1973).

[48] E. Piña, Dinámica de Rotaciones (UAM-Izt., México D. F. 1996).

[49] H. Risken, The Fokker-Planck Equation (Springer-Verlag, New York 1984).

[50] R. Roy, A. W. Yu and S. Zhu, Phys, Rev. Lett. 55, 2794 (1985).

[51] H. E. Stanley, Introduction to Phase Transitions and Critical Phenomena (Oxford University Press, 1971). 
[52] J. M. Sancho and M. San Miguel, Z. Phys. B36, 357 (1980).

[53] J. M. Sancho and M. San Miguel, Phys. Rev. A39, 2722 (1989).

[54] M. Suzuki, Order and Fluctuations in Equilibrium and Nonequilibrium Statistical Mechanics (Eds. G. Nicolis, G. Gewel and J. W. Turner, Wiley 1980).

[55] M. Suzuki, Y. Liu and T. Tsumo, Physica A138, 743 (1986).

[56] H. L. Swinney and J. P. Gollub, Hydrodynamic Instabities and the Transition to Turbulence (Spring Verlag, 1981).

[57] R.L. Stratonovich, Topics in the Theory of Ramdom Noise, Vol. I (Gordon and Breach, New York 1963) and vol. II (Gordon and Breach, New York 1967).

[58] M. C. Torrent and M. San Miguel, Phys. Rev. A38, 245 (1988).

[59] M. C. Torrent, F. Sagues and M. San Miguel, Phys. Rev. A40, 6662 (1989).

[60] N.G. Van Kampen, Stochastic Processes in Physics and Chemistry (Nort-Holland, Amsterdam 1981).

[61] G. Vemuri and R. Roy, Phys. Rev A39, 2539 (1989).

[62] G. Vemuri and R. Roy, Optics Comm. 77, 318 (1990).

[63] N. Wax, Noise and Stochastic Processes (Dover, New York 1954).

[64] S. Zhu, A.W. Yu and R. Roy, Phys. Rev A34, 4333 (1986). 